Portland State University

PDXScholar

$1-14-2022$

\title{
Development of a Testing Station for Grid-Interactive Smart Inverter Systems
}

Yudong Lan

Portland State University

Follow this and additional works at: https://pdxscholar.library.pdx.edu/open_access_etds

Part of the Power and Energy Commons

Let us know how access to this document benefits you.

\section{Recommended Citation}

Lan, Yudong, "Development of a Testing Station for Grid-Interactive Smart Inverter Systems" (2022). Dissertations and Theses. Paper 5892.

https://doi.org/10.15760/etd.7763

This Thesis is brought to you for free and open access. It has been accepted for inclusion in Dissertations and Theses by an authorized administrator of PDXScholar. Please contact us if we can make this document more accessible: pdxscholar@pdx.edu. 
Development of a Testing Station for Grid-Interactive Smart Inverter Systems

by

Yudong Lan

A thesis submitted in partial fulfillment of the requirements for the degree of

\author{
Master of Science \\ in \\ Electrical and Computer Engineering
}

Thesis Committee:

Robert Bass, Chair

Mahima Gupta

John M. Acken

Portland State University

2021 
(C) 2021 Yudong Lan 


\begin{abstract}
As renewable energy penetration increases within the electrical power grid, flexible power is required to ensure reliability. Reliability can be jeopardized by the fluctuation and nondispatch ability of renewable generators. This instability is caused by an imbalance between the amount of power produced and the amount of power consumed. Grid-enabled battery inverter systems are considered a solution to offset voltage and frequency fluctuations, which arise due to this power imbalance.

The objective of this work is to develop a system for testing grid-enabled inverters. These inverters use one or more communication standards to control their interactions with the grid. This testing system verifies bis functionality before utilities deploy them to provide grid services. The system contains three portions. One is the hardware components of the system. Next is the information exchange using communication protocols. The third is the testing procedures that check if the bis does what is expected when it receives control commands.
\end{abstract}


Dedication

For my parents and my girlfriend who always believe and support me 


\section{Acknowledgements}

Throughout the entire research period, I received so much support from my professor, family, friends, and my girlfriend. Without their help and support, I would not have finished my thesis research.

I would like first thank my professor, Dr. Robert Bass, for allowing me to join the research group. His patience, guidance, and experience helped me to be a qualified researcher. I also want to thank a Ph.D. Tylor Slay. He gave me so much help with program writing, interacting between hardware and software, and troubleshooting with Raspberry Pi.

The people I want to thank the most are my parents, Zhimei Wang and Wenzhao Lan. Without their support, I would not have had the opportunity to study abroad nor would I have had a chance to improve myself. My appreciation also goes to my girlfriend, Jihong Guo. She accompanied me during the pandemic and my research period. Without her, I cannot imagine how I would have lived through this time.

A special thanks go to my friend Shahad Alomani. She provided precious suggestions and guidelines to my thesis writing. It is her help that enabled me to do a better job on my thesis writing. 


\section{Contents}

$\begin{array}{ll}\text { Abstract } & \text { i }\end{array}$

Dedication $\quad$ ii

Acknowledgements

List of Tables $\quad$ vii

List of Figures $\quad$ ix

Acronyms $\quad$ xii

1 Introduction 1

1.1 Problem Statement . . . . . . . . . . . . . . . . . . 1

1.2 Objectives of Work . . . . . . . . . . . . . . 2

2 literature review 3

2.1 Distributed Energy Resources . . . . . . . . . . . . . . . . . 3

2.2 Battery Storage . . . . . . . . . . . . . . . . . . . . . . . . . . .

2.2.1 Battery Storage Trend . . . . . . . . . . . . . . 4

2.2.2 Applications ..................... 5

2.3 Smart Inverter . . . . . . . . . . . . . . . . . . . 5

2.4 California Rule $21 \ldots \ldots \ldots \ldots$

2.4 .1 Phase $1 \ldots \ldots \ldots \ldots \ldots$

2.4 .2 Phase $2 \ldots \ldots \ldots \ldots$

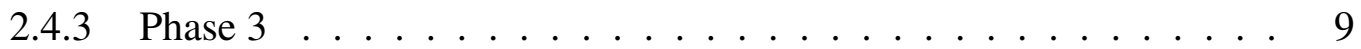

2.5 IEEE $2030.5 \ldots \ldots \ldots \ldots \ldots$

2.6 Use of IEEE 2030.5 between Server and Client $\ldots \ldots \ldots \ldots$

3 Smart Inverter Test Station $\quad 13$

3.1 Smart Inverter . . . . . . . . . . . . . . . . . . . . . . . . . . . . . . . . . . .

3.2 Battery Storage System . . . . . . . . . . . . . . . 16

3.3 Grid Simulator . . . . . . . . . . . . . . . . . . . . . . 18

3.4 PA 4000 Power Analyzer . . . . . . . . . . . . . . . . . . . . . 19

3.5 TDS2000B Digital Storage Oscilloscope . . . . . . . . . . . . . . 19 
4 DER Program Development $\quad 20$

4.1 DER attributes . . . . . . . . . . . . . . . . . . . 21

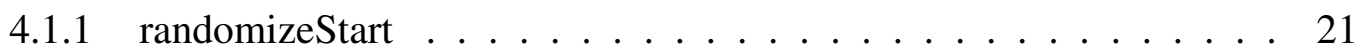

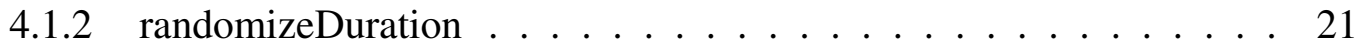

4.1 .3 opModEnergize . . . . . . . . . . . . . . . . 22

4.1 .4 opModFixedW ....................... 22

4.1 .5 DateTimeInterval . . . . . . . . . . . . . . 22

4.1 .6 opModVoltVar ...................... 22

4.1 .7 opModFreqWatt . . . . . . . . . . . . . . . . 23

4.2 DER Program Overview . . . . . . . . . . . . . . . . 23

4.3 DER Control Overview . . . . . . . . . . . . . . . . . . . . . . . 24

4.4 DER Curve Overview . . . . . . . . . . . . . . . . . 25

4.5 Testing Program of DER-Inverter . . . . . . . . . . . . 26

5 Testing procedure $\quad 27$

5.1 Check battery level . . . . . . . . . . . . . . . . . . 27

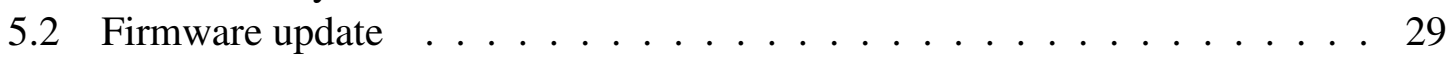

5.2.1 Firmware checking . . . . . . . . . . . . . . 29

5.2 .2 Firmware Update . . . . . . . . . . . . . . . . . 30

5.2.3 GS8048A firmware update . . . . . . . . . . . . . . . . 32

5.3 Setting the Grid Simulator . . . . . . . . . . . . . . . 33

5.3.1 Change configuration of grid simulator NHR 9410 . . . . . . . . 34

5.3.2 Change settings of grid simulator using the software NHR 9400 panel 35

5.4 Setting the GS8048A smart inverter . . . . . . . . . . . . 37

5.4 .1 Turn on the inverter . . . . . . . . . . . . 37

5.4.2 Enable module GS8048A and GS4048A . . . . . . . . . . . . . 38

5.4 .3 Enable Grid-Tied Mode . . . . . . . . . . . . . . . . . . . . 39

5.4.4 Change Relay Operation Time . . . . . . . . . . . . . . . . 40

5.4 .5 Selling power to grid ... . . . . . . . . . . . . 41

5.5 Identify IP address of AXS port . . . . . . . . . . . . . . . . . . . . . . . . . . . 41

5.6 Charging Battery . . . . . . . . . . . . . . . . . 42

5.7 Remote Development of the DER program on the Raspberry Pi . . . . . . . 43

5.8 Measuring output parameters from inverter . . . . . . . . . . . . . . 45

5.9 Checking SunSpec Registers Status . . . . . . . . . . . . . . . . 47

5.9.1 Using modbus poll software . . . . . . . . . . . . . . 47

5.9 .2 Using Outback Shell program . . . . . . . . . . . . . . . . . . . 49

5.9 .3 Running DER program . . . . . . . . . . . . . . . 50

6 Results \& Analysis $\quad 51$

6.1 Verify The Values Written Into The SunSpec Registers . . . . . . . . . . 51

6.2 Energize and De-energize Testing . . . . . . . . . . . . . 53

6.2.1 Energize and De-energize Testing Analysis . . . . . . . . . . 54

6.3 Maximum Export Power Testing . . . . . . . . . . . . . . . . . 55 
6.3.1 Maximum Export Power interoperability Testing Analysis . . . . . 56

6.3.2 Maximum Power Export Testing Connected with Grid Simulator . . 57

6.3.3 Maximum Export Power Sub-component Testing Analysis . . . . . 59

6.4 Volt-VAr function Testing . . . . . . . . . . . . . . . 60

6.4.1 Verification of Writing Volt-VAr Curve Points . . . . . . . . . . 61

6.4.2 Volt-VAr function interoperability testing analysis . . . . . . . . 63

6.4.3 GS8048A Volt-VAr Function Testing with Grid Simulator . . . . . 63

6.4.4 Volt-VAr function Sub-component Testing Analysis . . . . . . . . . 76

6.5 Frequency Watt Function Testing . . . . . . . . . . . . . . . . 77

6.5.1 Verification of Writing Frequency-Watt Curve Points . . . . . . . . 79

6.5.2 Frequency-Watt function interoperability testing analysis . . . . . 81

6.5.3 Frequency-Watt Sub-component testing preparation . . . . . . . . . 82

6.5.4 GS8048A Frequency-Watt Function Testing with Grid Simulator . . 83

6.5.5 Frequency-Watt function Sub-component Testing Analysis . . . . . 97

7 Conclusion $\quad 98$

$\begin{array}{ll}\text { Bibliography } & 100\end{array}$ 


\section{List of Tables}

3.1 Voltage reference points and time for battery charging. . . . . . . . . . 17

4.1 Mapping IEEE 2030.5 to SunSpec register. . . . . . . . . . . . . . . . . 26

5.1 SunSpe registers as shown in Modbus Poll. . . . . . . . . . . . . . . . 48

6.1 Result of writing and transferring IEEE 2030.5 "opmodConnect" to SunSpec "Conn" . . . . . . . . . . . . . . . . . . . . 53

6.2 Verify energize and de-energize numbers were transferred into SunSpec registers using OutBack Shell Program . . . . . . . . . . . . . . . . . . 53

6.3 Verify Energize or De-energize of an inverter through observing the voltage and current changes . . . . . . . . . . . . . . . . . 54

6.4 Test result of verifying connection and setting maximum power of the inverter. . 55

6.5 Using DER program to set curve points for Volt-VAr function. . . . . . . . . . 61

6.6 Verify Volt-VAr points were transferred into SunSpec registers using OutBack Shell program. . . . . . . . . . . . . . . . . . . . . 62

6.7 Default Volt-VAr curve points. . . . . . . . . . . . . . . . . . . . . . . 64

6.8 Default Volt-VAr curve points. . . . . . . . . . . . . . . . . . . . . . 65

6.9 Minimum Volt-VAr curve points. . . . . . . . . . . . . . . . . . . 68

6.10 Minimum Volt-VAr curve points. . . . . . . . . . . . . . . . . . . . 69

6.11 Maximum Volt-VAr curve points. . . . . . . . . . . . . . . . . . . 72

6.12 Maximum Volt-VAr curve points. . . . . . . . . . . . . . . . . 73

6.13 Using DER program to write values into SunSpec registers $\mathrm{Hz} 1$ to $\mathrm{Hz} 4$. . . 80

6.14 Using DER program to write values into SunSpec registers W1 to W4. . . . . . 80

6.15 Verify frequency-Watt points were transferred into SunSpec registers using OutBack Shell program. . . . . . . . . . . . . . . . . . 81

6.16 Control parameters of the Freq-Watt function in GS 8048A smart inverter . . 82

6.17 Frequency-Watt curve points with $58 \mathrm{~Hz}$ and $61 \mathrm{~Hz}$ sweep from the minimum to the maximum frequency. . . . . . . . . . . . . . . . 84

6.18 Frequency-Watt curve points with $58 \mathrm{~Hz}$ and $61 \mathrm{~Hz}$ sweep from the maximum to the minimum frequency. . . . . . . . . . . . . . . . 85

6.19 Frequency-Watt curve points with $58 \mathrm{~Hz}$ and $61 \mathrm{~Hz}$ sweep from the minimum to the maximum frequency. . . . . . . . . . . . . . . . . . 89

6.20 Frequency-Watt testing $(61 \mathrm{~Hz}-55 \mathrm{~Hz})$ with 2 ss and maximum power capacity 90 
6.21 Frequency-Watt testing $(59.5 \mathrm{~Hz}-65 \mathrm{~Hz})$ with 2 seconds time step and half power capacity . . . . . . . . . . . . . . . . 93

6.22 Frequency-Watt testing $(65 \mathrm{~Hz}-59.5 \mathrm{~Hz})$ with 2 seconds time step and full power capacity . . . . . . . . . . . . . . . . 95 


\section{List of Figures}

2.1 Large scale battery storage usage trend . . . . . . . . . . . . . . . . 4

2.2 Small scale battery usage trend . . . . . . . . . . . . . . . . . 5

2.3 Worldwide smart inverter usage trend . . . . . . . . . . . . 7

2.4 Utility to DER communication working algorithm . . . . . . . . . . . . 12

3.1 Smart inverter test station . . . . . . . . . . . . . . . . . . . . . 14

3.2 Radian series GS8048A smart inverter . . . . . . . . . . . . . . . 15

3.3 Mate3s . . . . . . . . . . . . . . . . . . . . 16

3.4 SunSpec Modbus interface . . . . . . . . . . . . . . . . . 16

3.5 Aqucous Hybrid Ion battery . . . . . . . . . . . . . . . . . . . . . . . . . . . . . . 17

3.6 NHR grid simulator . . . . . . . . . . . . . . . . . . . . . . . . . . . . . . . . . .

3.7 Power Analyzer . . . . . . . . . . . . . . . . . . . . . . . . . . . 19

3.8 Oscilloscope . . . . . . . . . . . . . . . . . . 19

4.1 DER Program flow chart . . . . . . . . . . . . . . . . . . 24

4.2 DER Control flow chart . . . . . . . . . . . . . . . . . 25

4.3 DER curve flow chart . . . . . . . . . . . . . . 25

5.1 MATE3s home screen . . . . . . . . . . . . . . . . . 27

5.2 Hot and neutral line of GS8048A smart inverter . . . . . . . . . . . . 28

5.3 Dr. Meter power supply . . . . . . . . . . . . . . . . . . . . . . . . . . . . . . . . . . . . .

5.4 MATE3s password . . . . . . . . . . . . . . . . . . 29

5.5 Firmware interface of MATE3s and GS8048A inverter . . . . . . . . . . 30

5.6 Power rack . . . . . . . . . . . . . . . . . . 33

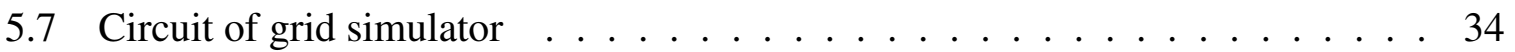

5.8 Circuit breaker on power rack . . . . . . . . . . . . . . . . 34

5.9 Neutral switch on power rack . . . . . . . . . . . . . . 35

5.10 NHR 9410 software interface . . . . . . . . . . . . . . . 35

5.11 Configuring grid simulator on NHR software $\ldots \ldots \ldots$

5.12 Power switch of GS8048A smart inverter . . . . . . . . . . . . . . 37

5.13 Module selection on GS8048A . . . . . . . . . . . . . . . . . . . . . . . . . . . . . . . . . . . .

5.14 Enable Grid Tied mode of GS8048A . . . . . . . . . . . . . . . . . . . 39

5.15 Enable Offset operation of GS8048A . . . . . . . . . . . . . . . . . . 40

5.16 Time delay settings for GS8048A . . . . . . . . . . . . . . . . . . . . . . . . . . . .

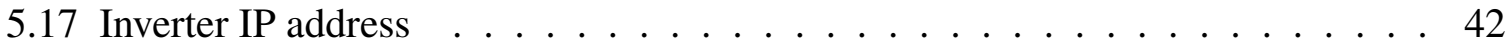


5.18 Battery voltage reference points . . . . . . . . . . . . . 43

5.19 Outback Shell . . . . . . . . . . . . . . . . . . . . . . 50

6.1 SunSpec blocks in AXS port . . . . . . . . . . . . . . . . . 52

6.2 Verify the maximum power written into the SunSpec register . . . . . . . . 56

6.3 Maximum active power export from inverter . . . . . . . . . . . . 57

6.4 Grid simulator absorb power from load . . . . . . . . . . . . . . . . 58

6.5 Grid simulator export power to load . . . . . . . . . . . . . . . . 58

6.6 Exporting half power from inverter . . . . . . . . . . . . . . 59

6.7 Standard Volt-VAr curve . . . . . . . . . . . . . . . . . . . . 60

6.8 Expected Volt VAr curve . . . . . . . . . . . . . . . . . . . . 62

6.9 Default Volt-VAr curve sweep voltage from the minimum to maximum with 2 seconds time step . . . . . . . . . . . . . . . . . . . . 64

6.10 Default Volt-VAr testing sweep voltage from high to low with 2 seconds time step 66

6.11 Default Volt-VAr testing sweep voltage from low to high with 5 seconds time step 67

6.12 Default Volt-VAr testing sweep voltage from high to low with 5 seconds time step 67

6.13 Minimum Volt-VAr testing sweep voltage from low to high with 2 seconds time

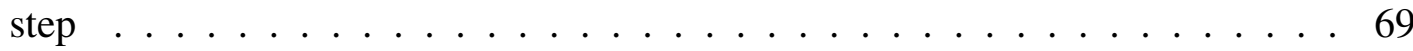

6.14 Minimum Volt-VAr testing sweep voltage from the high to low with 2 seconds time step . . . . . . . . . . . . . . . . . . . 70

6.15 Minimum Volt-VAr testing sweep voltage from low to high with 5 seconds time

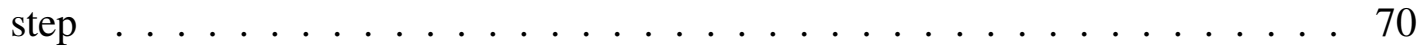

6.16 Minimum Volt-VAr testing sweep voltage from high to low with 5 seconds time

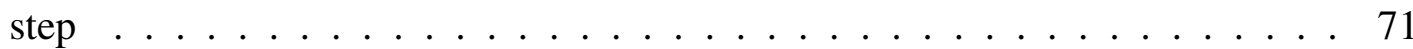

6.17 Maximum Volt-VAr testing sweep voltage from low to high with 2 seconds time step . . . . . . . . . . . . . . . . . . . . 73

6.18 Maximum Volt-VAr testing sweep voltage from high to low with 2 seconds time step . . . . . . . . . . . . . . . . . . . . . . 74

6.19 Maximum Volt-VAr testing sweep voltage from low to high with 5 seconds time step . . . . . . . . . . . . . . . . . . . . . 74

6.20 Maximum Volt-VAr testing sweep voltage from high to low with 5 seconds time step . . . . . . . . . . . . . . . . . . 75

6.21 Frequency-Watt curve for over-frequency . . . . . . . . . . . . . . 77

6.22 Bi-directional Frequency-Watt Curve . . . . . . . . . . . . . . . . 78

6.23 User-defined expected bi-directional Frequency-Watt curve. . . . . . . . . . . . 79

6.24 Frequency-Watt testing $(58 \mathrm{~Hz}-61 \mathrm{~Hz})$ with 2 seconds time step and half power capacity . . . . . . . . . . . . . . . . . . . 84

6.25 Frequency-Watt testing $(61 \mathrm{~Hz}-58 \mathrm{~Hz})$ with 2 seconds time step and maximum power capacity . . . . . . . . . . . . . . . . 86

6.26 Frequency-Watt testing $(58 \mathrm{~Hz}-61 \mathrm{~Hz})$ with 5 seconds time step and maximum power capacity . . . . . . . . . . . . . . . . . . . . 87

6.27 Frequency-Watt testing $(61 \mathrm{~Hz}-58 \mathrm{~Hz})$ with 5 seconds time step and maximum

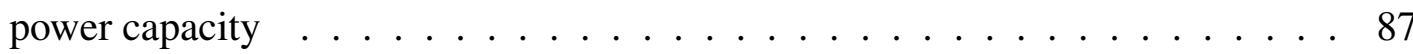


6.28 Frequency-Watt testing $(55 \mathrm{~Hz}-61 \mathrm{~Hz})$ with 2 seconds time step and maximum power capacity . . . . . . . . . . . . . . . . . . . . 89

6.29 Frequency-Watt testing $(55 \mathrm{~Hz}-61 \mathrm{~Hz})$ with 2 seconds time step and maximum power capacity . . . . . . . . . . . . . . . . . . . 90

6.30 Frequency-Watt testing $(55 \mathrm{~Hz}-61 \mathrm{~Hz})$ with 5 seconds time step and maximum power capacity . . . . . . . . . . . . . . . . . 91

6.31 Frequency-Watt testing $(61 \mathrm{~Hz}-55 \mathrm{~Hz})$ with 5 seconds time step and maximum power capacity . . . . . . . . . . . . . . . . . 92

6.32 Frequency-Watt testing $(59.5 \mathrm{~Hz}-65 \mathrm{~Hz})$ with 2 seconds time step and half power capacity . . . . . . . . . . . . . . . . 94

6.33 Frequency-Watt testing $(65 \mathrm{~Hz}-59.5 \mathrm{~Hz})$ with 2 seconds time step and full power capacity . . . . . . . . . . . . . . . . . 95

6.34 Frequency-Watt testing $(59.5 \mathrm{~Hz}-65 \mathrm{~Hz})$ with 5 seconds time step and full power capacity . . . . . . . . . . . . . . . 96

6.35 Frequency-Watt testing $(65 \mathrm{~Hz}-59.5 \mathrm{~Hz})$ with 5 seconds time step and full power capacity . . . . . . . . . . . . . . . . . 97 


\section{Acronyms}

AHI Aquion Hybrid Ion

AXS Axis Port Gateway

BIS Battery Inverter System

CSIP Common Smart Inverter Profile

DCM Distributed Control Module

DER Distributed Energy Resource

DERMS Distributed Energy Resource Management System

DHCP Dynamic Host Configuration Protocol

EGoT Energy Grid of Things

EIA Environmental Impact Assessment

EVSE Electrical Vehicle Service Equipment

FSA Function Set Assignment

FTP File Transfer Protocol

HTTP Hypertext Transfer Protocol

HVAC Heating Ventilation and Air Conditioning

IDE Integrated Development Environment

IEC International Electrotechnical Commission

IEEE Institute of Electrical and Electronics Engineers

IP Internet Protocol

ISOs Independent System Operators 
LAN Local Area Network

MAC Media Access Control

NHR NH Research

RTOs Regional Transmission Organizations

SIWG Smart Inverter Working Group

TCP Transmission Control Protocol

UPS Uninterruptible Power Supply

UTC Coordinated Universal Time

XML Extensible Markup Language 


\section{Introduction}

\subsection{Problem Statement}

The electrical grid must have the capacity to transfer all the power generated from the power plant to satisfy load demand. However, load demand varies vastly during a day, and generators face a big challenge in the peak-to-base spread to match this load demand [1]. Additionally, around $20 \%$ of generation capacity needs to be provided in case of an unpredicted real power increase in load demand because of uncertainty in generation availability [2]. Moreover, reliability decreases as more non-dispatchable renewable energy becomes involved in the power grid. The output power from renewable generators fluctuates a lot because of weather and environmental conditions. Renewable generation is also non-dispatchable; it is only available when weather conditions are right. This could lead to an unstable grid frequency [3]. Therefore, Battery Inverter System (BIS)s are being considered as a solution that can help address the above problems. BISs are capable of quickly responding to grid demand, and provide energy storage to continue supply power for a period of time [4]. However, in order to dispatch BIS, inverters must be able to communicate with a Distributed Energy Resource Management System (DERMS). To do this, BIS use communication protocols such as Institute of Electrical and Electronics Engineers (IEEE) 2030.5 and Modbus SunSpec. And, the information exchange between 
the BIS and DERMS must be functionally correct.

\subsection{Objectives of Work}

Grid operators can not control millions of Distributed Energy Resources (DERs) individually, so DERMSs are used to aggregate many DERs. A DERMS is a software platform that is able to dispatch a very large number of DERs simultaneously and automatically, such as BIS, Electrical Vehicle Service Equipment (EVSE), and residential appliances through information exchange with a controller installed on each DER [5]. In this research, the DER of concern are BISs. However, to successfully dispatch BISs before they interact with an electric grid, there must be a communication bridge. For instance, the communication standard for a DERMS maybe IEEE 2030.5 while the standard for a BIS maybe SunSpec Modbus. The information exchange between the DERMS and BIS must be functionally correct, which means the BIS must correctly perform a specified action for each message it receives.

Therefore, the objective for this thesis is to create a testing system for grid-enabled BIS to test this functionality. This system tests grid-enabled BIS working under different communication protocols to ensure the BIS does what is expected when it receives a resource message from a DERMS. This testing must be done before a BIS can be deployed in an electric power system. The functional testing conducted in this research demonstrates the ability of this system to test grid-enabled BIS. 


\section{2 literature review}

\subsection{Distributed Energy Resources}

DERs are customer-owned generation, storage, and load assets that are grid-enabled. These resources are located behind a customer meter and are not traditionally directly managed by utilities. DER are usually small in size and support modularity. Generally, the capacity of DER is less than 10 kilowatts $(\mathrm{kW})$. Wind turbines, photovoltaics (PV), fuel

cells, and energy storage systems are the most widely applied generation DER. Storage DER are usually BIS. Load DER include HVAC systems, electric water heaters, EVSE and so on. Utilities are interested in using DER to reduce load or add power generation in order to maintain the stability of the power grid by reducing peak load demand, regulating frequency, and regulating voltage [6].

\subsection{Battery Storage}

As an increasingly adopted energy storage system, battery storage systems are deployed on both the utility-scale and the residential scale. To better understand the usage situations and applications of a battery storage system, the following subsections are provided. 


\subsubsection{Battery Storage Trend}

Battery Inverter System include low-power uses such as installed in a home or as highpower resources for a utility to support the grid. As reported by the EIA in the United States, the amount of power capacity added to the grid between 2003 and 2010 from largescale battery storage system was only $59 \mathrm{MW}$ in the US [7]. However, this situation has completely changed since 2011. As of 2018, there was 869 MW of power capacity installed in the form of large-scale BIS [7]. Moreover, there was a rapid large-scale power installation increase between 2015 and 2018, as shown in Figure 2.1. Independent System Operators (ISOs) and Regional Transmission Organizations (RTOs) are major users of large-scale BIS

\section{[7].}

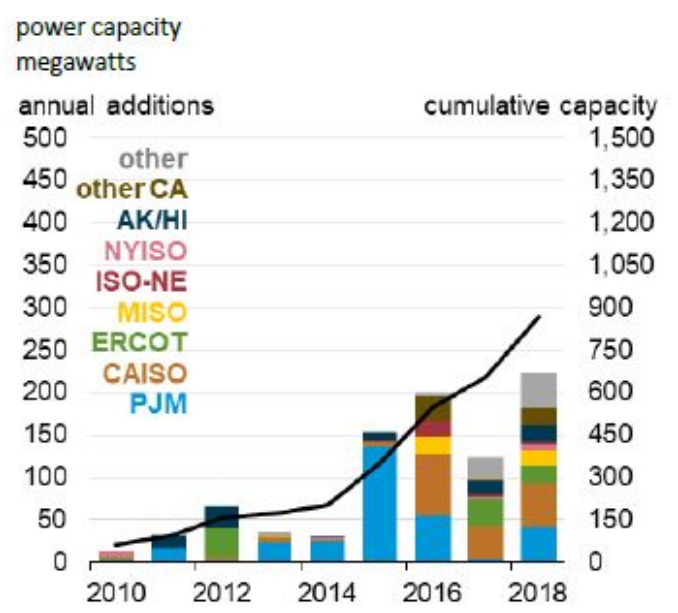

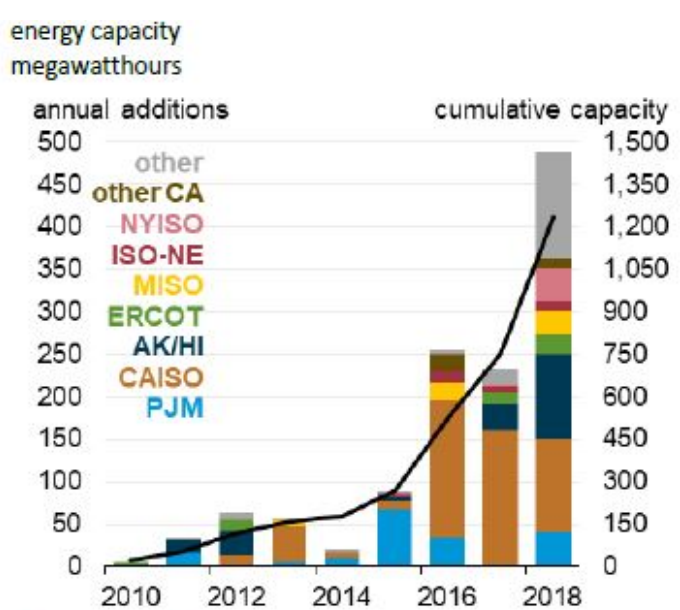

Sources: U.S. Energy Information Administration, Form EIA-860M, Preliminary Monthly Electric Generator Inventory; U.S. Energy Information Administration, Form EIA-860, Annual Electric Generator Report

Figure 2.1: Large-scale battery storage capacity by region (2003-2018) [7].

Not only large-scale battery storage system, but small-scale battery storage power also has a bright future. As reported in 2018, the power capacity of small-scale battery storage was 234 MW in the US [7]. More than $50 \%$ of this capacity was used for commercial purposes, 
$31 \%$ power was installed for residences, $15 \%$ used in industry, and $3 \%$ was connected to the distribution grid. Small-scale battery usage in the residential and commercial fields has grown quickly in recent years and is being deployed in many states in the U.S., as shown in Figure 2.2.
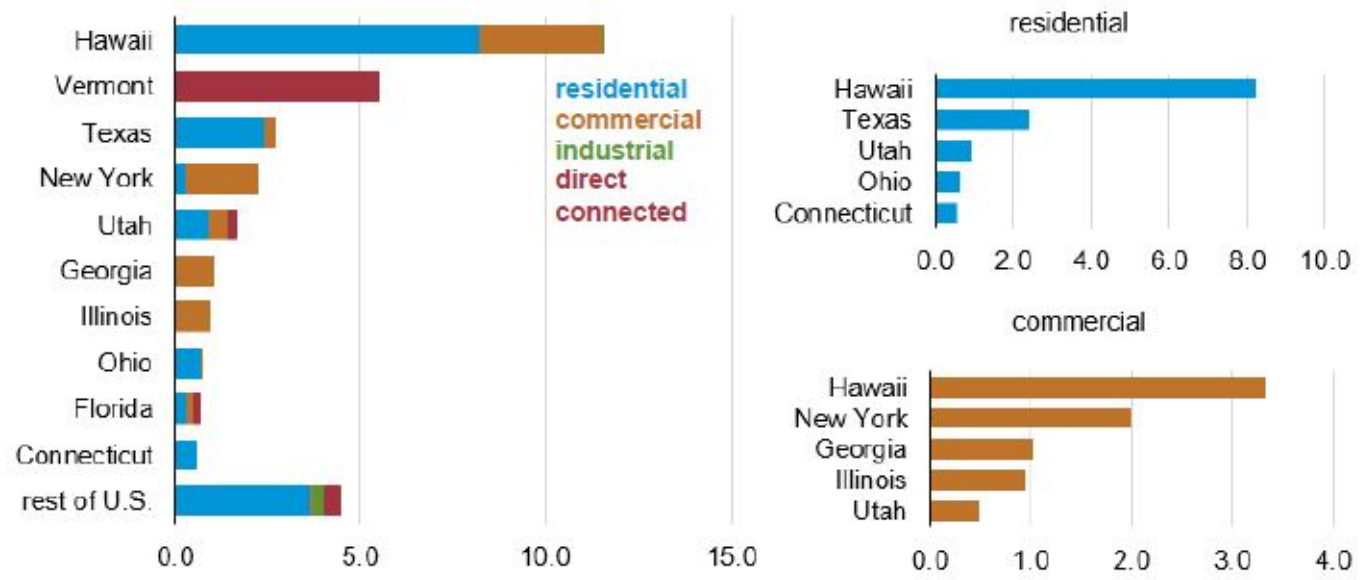

Source: U.S. Energy Information Administration, Form EIA-861, Annual Electric Power Industry Report

Figure 2.2: The usage purpose and location of small-scale battery storage systems in the US [7].

\subsubsection{Applications}

Current utility-scale BIS provide grid-scale services such as peak load shifting, frequency and voltage regulating, excess power storing, and so on. However, grid-interactive smallscale BIS are also under development. Small-scale batteries deployed in residence and commercial locations provide power outage protection and optimize energy usage.

\subsection{Smart Inverter}

Unlike a traditional inverter that simply feeds power into the grid, a smart inverter can dynamically work with the grid to increase grid reliability and stability. In orther words, a 
smart inverter can autonomously make decisions to help maintain reliability. To achieve autonomous control, a smart inverter must be programmable to allow precise control of its input and output power as well as its ramp rate. In addition, a smart inverter should also be capable of grid-support functions and have the ability for bidirectional communication. As more DERs equipped with grid-support functionality become avaliable, utilities can work with them to compensate the grid. These DERs can be valuable assets instead of a burden to the grid [8].

When connected to DERMS, smart inverters can instruct BIS to make react in responds to message from a utility to help solve problems of grid abnormalities. Moreover, DERMS can reprogram smart inverters in real-time to make sure the inverters always have the best response to anticipated events on the electric grid [9]. By offering automatic response to electric grid disturbances, smart inverters have become more widely adopted in recent years, as shown in the graph in Figure 2.3

\subsection{California Rule 21}

CA Rule 21 specifies smart inverter requirements. It was created based on the recommendations of the Smart Inverter Working Group. It has three phase of implementations:

- Phase 1: Autonomous function implementation of smart inverters

- Phase 2: Choose and apply communication requirement to smart inverters

- Phase 3: Add advanced functions to smart inverters 


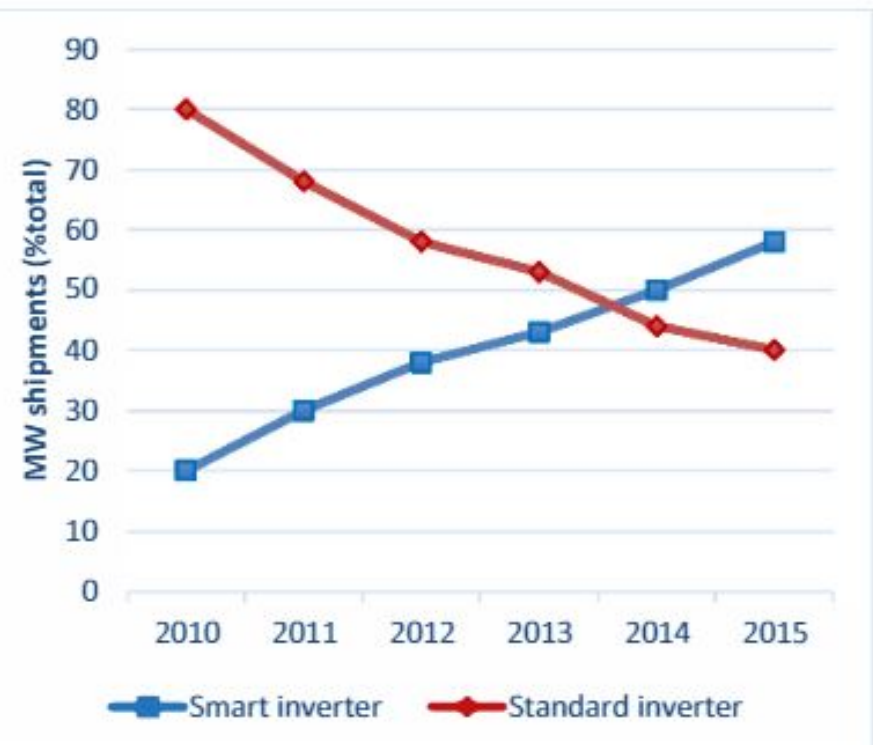

Figure 2.3: World market share of standard inverters and smart inverters[10].

\subsubsection{Phase 1}

Utilities in each state in the U.S. have different interconnection policies, so some settings of smart inverters have to be configured differently in order to conform to the utilities' interconnection handbook. Once the settings are configured, the smart inverter can be programmed to autonomously adjust the output based on the local conditions, thereby keeping the grid stable and reliable [11]. The functions and settings that need to be configured in smart inverters are:

- Low/High Voltage Ride-through: The inverter must not trip (i.e. disconnect from the grid), during AC voltage excursions outside the normal operating range, during a specified time duration. It must either cease output momentarily, or continue outputting power, depending on the severity of the voltage deviation, while sustaining 
its electrical connection to the grid. The inverter must trip for time durations longer than the ride-through times specified [12].

- Low/High Frequency Ride-through: The inverter must continue to output power to the grid during AC frequency excursions of specified duration and deviation from the normal operating frequency of $60 \mathrm{~Hz}$. The inverter must trip for time durations longer than the specified ride-through times [12].

- Volt-VAr Function: This function allows smart inverters to dynamically respond to the grid voltage fluctuation by supplying or absorbing VAr to help stabilize the grid.

- Ramp Rate: The rate of change in the output power of the inverter

- Fixed Power Factor: Provide certain reactive power by setting a fixed power factor

- Soft Reconnect: Provide "soft-start" methods

- Anti-Islanding: Trip off inverter under long term abnormal condition

\subsubsection{Phase 2}

In order to exchange information between smart inverters and DERMS, a communication protocol must be selected. According to Gordon Lum: "This protocol must operate over Transmission Control Protocol (TCP)/Internet Protocol (IP); must operate over different media interfaces; use the international standard International Electrotechnical Commission (IEC) 61850 as the information model; provide cybersecurity at the transport and application layers, and provide cyber-security for user and device authentication" [11]. Based on the listed restriction above, IEEE 2030.5 was selected as one of three default communication standards. Within IEEE 2030.5, direct control, autonomous curve control, 
and other capabilities of DER are well defined. These attributes ensure DERMS can dispatch DERs based on utilities' needs to stabilize the grid.

To describe how one would implement IEEE 2030.5 to satisfy the requirements described in CA Rule 21 Phase 2, a guideline called the Common Smart Inverter Profile (CSIP) was created. The following are some guiding principles of CSIP [13]:

- All smart inverters require communications to achieve their full value as DER

- To achieve complete interoperability, a complete profile is required, including a data model, a messaging model, a communication protocol, and security. Without a complete profile specification it would be impossible to achieve communications interoperability without additional systems integration activities

- Leverage existing standards and models from both engineering (e.g., IEEE 1547) and communications (e.g., IEEE 2030.5) standards. The development of a new, standalone standard would create additional burdens for all parties and only serve to raise costs of both development and maintenance.

\subsubsection{Phase 3}

Advanced functions added to smart inverters will assist in improving power system reliability by reducing effects caused by disturbances, and increase energy efficiency. The following are some advanced functions that the Smart Inverter Working Group (SIWG) completed analysis in smart inverters [14]: 
- Limit maximum real power mode: This function provides a maximum real power limit that a DER client can produce or output to a electrical connection points such as PCC or an aggregation system.

- Set Real power mode: This function defines a real power value that a DER client can produce or output to a electrical connection points.

- Provide a Frequency-Watt Emergency mode: This mode allows the frequency-watt function to increase or decrease power when the grid frequency is unstable. Also, this mode becomes a controllable setting, which means the frequency-watt function can be either enabled or disabled when needed. The rate of the active power change shall be settable as well.

- Provide a Volt-Watt mode: This function try to deal with the issue such as High/Low voltage ride through, localized high service voltage and high penetration of DER systems at the distribution level.

- Provide a dynamic reactive current support mode

- Allow scheduling of power values and modes

- Allow for DER functions that are also important to DER integrators and other third parties

\subsection{IEEE 2030.5}

The IEEE 2030.5 communication standard was developed from 2013 through 2018 based on the Common Information Model (CIM), with the goal of creating a message-based commu- 
nication standard for the modern grid, distributed smart devices, and interoperability [15]. It adopted a wide-range of communication protocols, such as TCP/IP and Hypertext Transfer Protocol (HTTP), so DER devices can be managed either individually or in aggregate between utilities and end-users [16]. In IEEE 2030.5, the communication messages between a DERMS server and DER clients uses Extensible Markup Language (XML) schema, as defined in IEEE 2030.5 XML Schema. Typically, the server exposes the available resource to the client through hosting the related URL and the client will GET or PUT/POST the updated information back to the resource [17].

\subsection{Use of IEEE 2030.5 between Server and Client}

The general working algorithm between the DERMS server (or Aggragator) and DER clients is as follow: the server send the control information to the DER client then DER client send information back to the server after executing the control. The DER controls sent from Server to DER devices include [17]:

- Autonomous control: Volt-VAr, Freq-Watt, and Volt-Watt curve based control

- Protection settings: High/Low Voltage Ride-Through, High/Low Frequency Ride through

- Immediate controls: active power curtailment, fixed reactive power, fixed power factor

The information sent from DER client to server include [17]:

- DER nameplate ratings and settings 
- DER alarms and status

- DER measurements (W, VAr, V, A, PF, Wh, Hz, etc.)

Fig 2.4 shows a diagram of the communication options between the server and DER clients. Option $\mathbf{C}$ is adopted in this research to show if the inverter provides a functionally correct response to the IEEE 2030.5 messages sent from a DER controller.

(a)

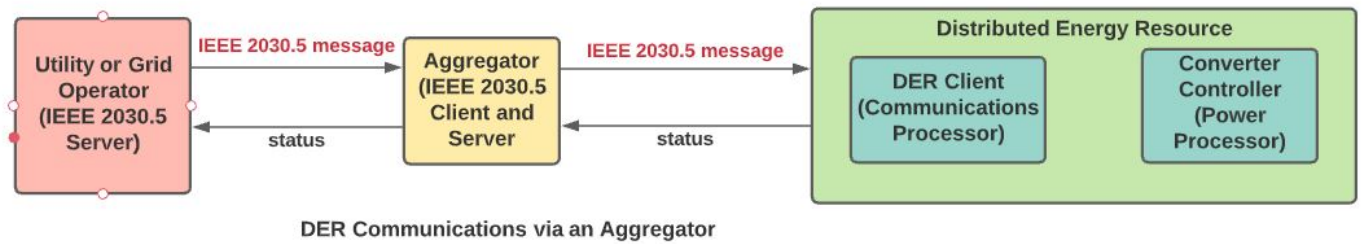

(b)

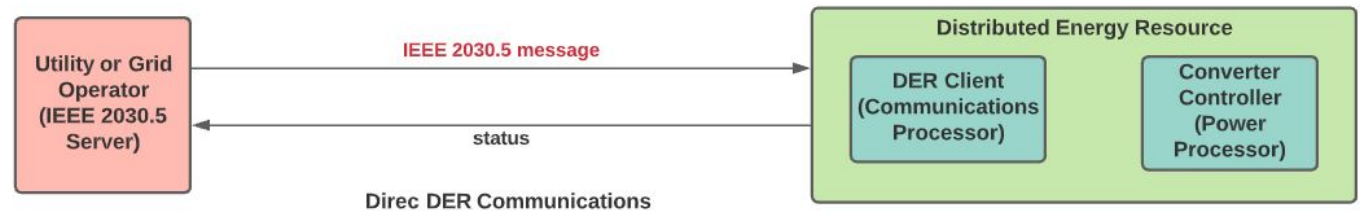

(c)

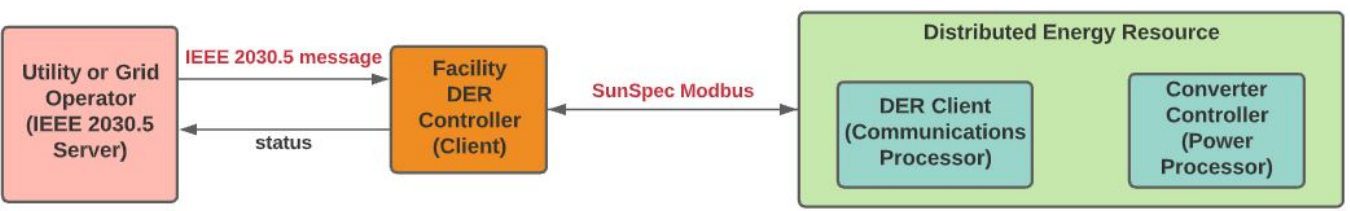

DER Communications via a Facility or Plant Controller

Figure 2.4: Utility-to-DER communications options per CA Rule 21 and CSIP [17]. 


\section{Smart Inverter Test Station}

The smart inverter test station has three main hardware components: a smart inverter, a battery, and a grid simulator. The smart inverter is the device under test. The battery and the grid simulator support the testing. The smart inverter coupled with a battery is called a BIS. When a controller is used to control the BIS, the whole system will be used as a DER. The controller mentioned here is a Raspberry Pi 3 Model B single-board computer. It communicates with the smart inverter through an Ethernet cable and communicates with DERMS via a Wi-Fi Local Area Network (LAN) in the Portland State University Power Lab. The Raspberry Pi and control software together are composed as a Distributed Control Module (DCM), which communicate with a DERMS. The DCM receives controls from the DERMS and converts them into SunSpec Modbus commands for the inverter. The grid simulator connects to the inverter through a power cord and is used for testing the grid-enabled BIS. The equipment connection of smart inverter test station is shown in Figure 3.1. 


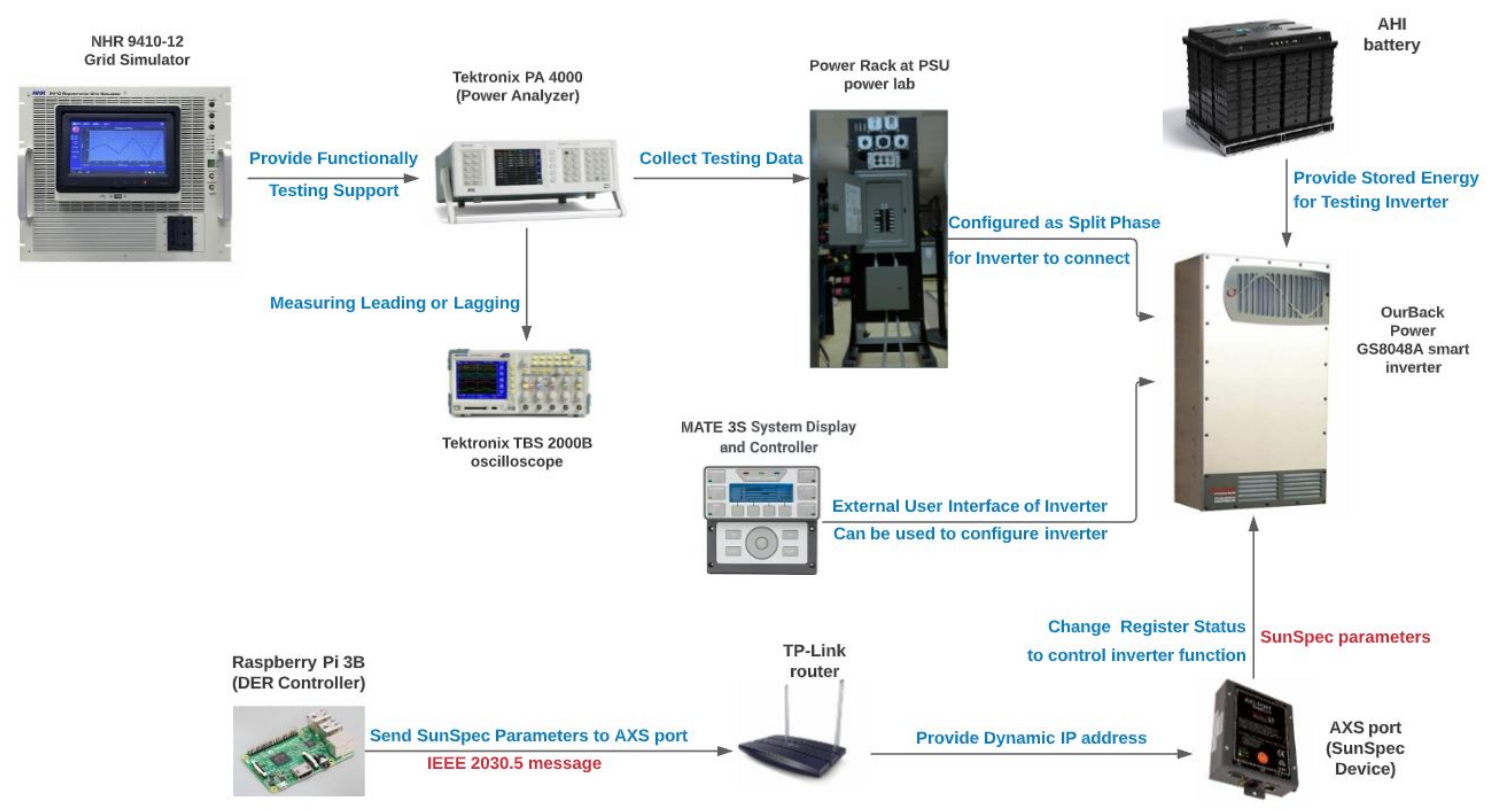

Figure 3.1: Equipment connection of the smart inverter test station.

\subsection{Smart Inverter}

The smart inverter used in this research is a Radian A-series inverter from OutBack POWER company. The model of this inverter is the Radian GS8048A. It has two modules. If only one module is enabled, the maximum output power is $4000 \mathrm{VA}$. If both modules are enabled, the maximum output power is 8000 VA. Seven AC input modes are avaliable in the inverter: Generator, Support, Grid Tied, Uninterruptible Power Supply (UPS), Backup, Mini Grid, and Grid Zero. Each mode has a different application. In this research, tests are conducted with the inverter in Grid Tied mode because this mode allows the inverter to be a grid-interactive device. Within this mode, the inverter not only absorbs power from the utility grid, but it can also supply power to the grid when excess battery energy is available. 


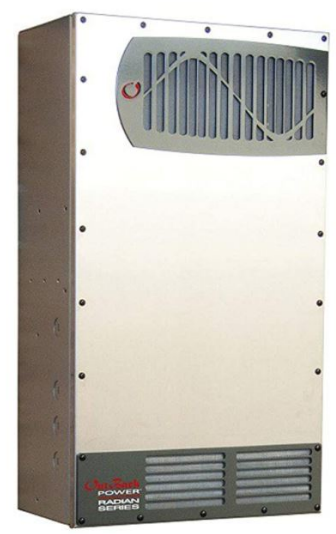

Figure 3.2: Outback Power GS8048A-01 Radian Series 8 kW Inverter [18].

Because the GS8048A does not have a user interface, an external control device is provided for the users to control it. The MATE3s is a system display and controller made by Outback Power. This controller has the ability to set multi-level passwords in case of unintended changes and also provides some necessary functions for user convenience.

Users can also use the Axis Port Gateway (AXS) Modbus TCP communication box to change SunSpec registers in order to set inverter functions. All SunSpec registers are contained in the AXS port. This device supports users to download system data using Modbus-read or File Transfer Protocol (FTP) transfer. Since the AXS is a TCP-supported device, users can set the SunSpec registers using modbus poll or outback shell program installed on the computer through finding the default IP address of the AXS or the IP address assigned by a router using Dynamic Host Configuration Protocol (DHCP).

In this research, both the MATE3s and AXS are all used. According to the GS8048A user manual, "the MATE3s must be used when upgrading the inverter to firmware revision 001.006.063 or higher [18]" 

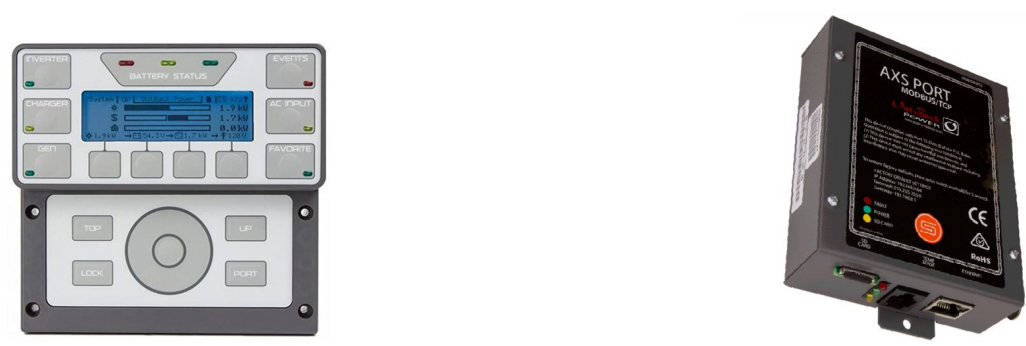

Figure 3.3: OutBack MATE3s System Controller Figure 3.4: AXS Modbus/TCP Interface Device [20]. [19].

\subsection{Battery Storage System}

The battery used in this research is an Aquion Hybrid Ion (AHI) battery. It has a long charge and discharge cycle life span and is designed to serve as a stand-alone system. The battery has twelve battery stacks and each stack can be either connected in parallel or series according to the different stationary applications [21].

Although the battery chemistry is AHI, the three-stage charging process for lead-acid batteries is applied: bulk, absorption, and float. The first charging stage is the bulk charge. In this stage, the battery voltage will increase as a constant current charge the battery. Usually, the battery will reach $75 \%$ to $90 \%$ of its capacity in this stage. Absorption charge is the second stage in the three-stage charging cycle. In this stage, voltage is constant and current varies to maintain the voltage level. At the end of this stage, the battery will reach $100 \%$ of its capacity. Float charge is the third stage of charging. Within this stage, the battery maintains $100 \%$ of storage capacity [18]. Otherwise, the inverter will slowly self-discharge. When different types of inverters connect to the AHI battery, some settings of three-stage charging are different. In this research, the smart inverter is the Radian Class Inverter, so the voltage settings and charging time settings are [21]: 


\begin{tabular}{|l|c|c|}
\hline Voltage Reference Point & Volts & Hour \\
\hline Bulk & 59.4 & N/A \\
\hline Re-bulk & 50 & N/A \\
\hline Absorb & 59.4 & $1 \mathrm{hr}$ \\
\hline Float & 54.4 & $4 \mathrm{hr}$ \\
\hline Re-float & 52 & N/A \\
\hline
\end{tabular}

Table 3.1: Voltage reference points and time for battery charging.

The re-bulk voltage is the voltage set point for the inverter to restart the charge cycle when battery voltage decreases due to discharge. The re-float charge is still in the float charge stage. Similar to the bulk charge, re-float is also a constant-current charge stage, but it is used to offset the battery self-discharge. Therefore, the charger will use re-float voltage as the set point when the battery voltage lower than this value [18].

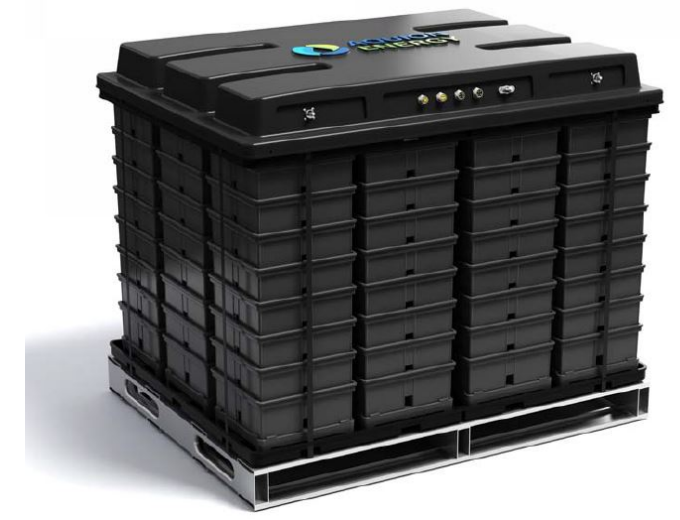

Figure 3.5: Aquion Energy Aqueous Hybrid Ion Battery [21]. 


\subsection{Grid Simulator}

The NH Research (NHR) 9410-12 grid simulator used in this research is a programmable four-quadrant bi-directional AC/DC power source. It supports grid-tied inverter testing and is capable of providing a wide range of frequencies and voltages. The frequency can be changed between $30 \mathrm{~Hz}$ and $100 \mathrm{~Hz}$. The output real power can be adjusted up to 12 $\mathrm{kW}$. The maximum apparent power that the simulator can handle is $33 \mathrm{kVA}$. The NHR 9410 includes a measurement system, so voltage, current, and power can be monitored in real-time. This grid simulator also supports multiple output power combinations: DC source, 1-phase, 2-phase, and 3-phase AC source [22]. Users can control per-phase magnitudes and waveform when different phases of AC source are under simulation.

In this research, the grid simulator is used for sweeping the voltage and frequency points to test the Volt-VAr and Frequency-Watt function of the inverter once the user-defined values are written into the relevant SunSpec registers.

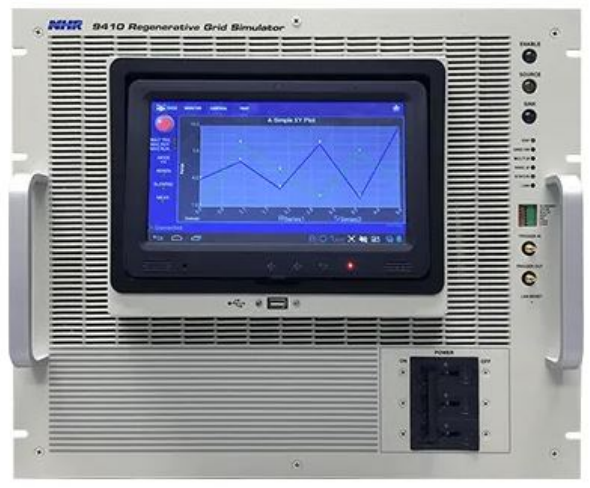

Figure 3.6: The Regenerative Grid Simulator NHR 9410 [22]. 


\subsection{PA 4000 Power Analyzer}

To collect the Volt-VAr, Frequency-Watt, and maximum exporting power testing results, a power analyzer is used. The PA 4000 power analyzer from Tektronix supports multiple channel measurements with high accuracy. It supports up to 30 A maximum current input, which matches the GS8048A smart inverter. Since the inverter is a split-phase unit, two channels are selected to use on the power analyzer to separately monitor the parameters in each phase.

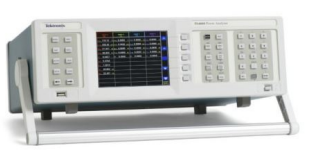

Figure 3.7: PA4000 Power Analyzer [23].

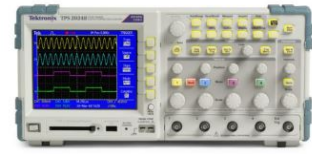

Figure 3.8: TPS2000B Digital Storage Oscilloscope [24].

\subsection{TDS2000B Digital Storage Oscilloscope}

To verify the reactive power is capacitive or inductive that the inverter provides or absorbs when the Volt-VAr function is enabled, an oscilloscope is used. The oscilloscope can show the leading or lagging refers to current and voltage. When the current leads the voltage, the reactive power is capacitive. In contrast, the reactive power is inductive when the current lags the voltage. The TDS2024C Digital Storage Oscilloscope is from Tektronix. It allows user to quickly and accurately measure a power system without too many operations. This oscilloscope supports four channels, so current and voltage in each phase of the inverter can be shown concurrently. 


\section{DER Program Development}

A BIS DER client is unable to be dispatched by a DERMS server when the communication standard is different, so a communication standard transformation bridge is necessary. The DER bridge program developed in this research relates the specific IEEE 2030.5 (standard of the Portland State University Power lab DERMS) attributes to corresponding SunSpec (standard of the Outback BIS) registers for inverter testing. In the DER program, two features of the inverter must be verified:

1. If the inverter can be energized at a specific time to export or import active power

2. If the Volt-VAr and Frequency-Watt curve points can be set using IEEE 2030.5 attributes and mapped to SunSpec registers in AXS port to enable the related function.

To test the above features, different DER attributes defined in IEEE 2030.5 are used, each of which are defined in the following section.

A DER control is based on an Event. The definition of an Event in IEEE 2030.5 is: "Any instance of a resource with a defined valid duration for which a client should take action. This would include all resources that are derived from the Event resource defined in the schema (IEEE Std 2030.5 supplemental material)" [25]. Briefly, an Event is an instance that has been sent from an aggregation server to a distributed client, which contains a scheduled 
start time and a finite time duration for a single device or a group of device to take action [26].

\subsection{DER attributes}

In IEEE 2030.5, many DER attributes are defined for client devices. For the testing system conducted in this research, five attributes are included. These attributes defines the operation time and output power of a smart inverter.

\subsection{1 randomizeStart}

The randomizeStart attribute defines a time span, in seconds, within which the client device can choose to start an Event any time in the range. For example, if the scheduled start time of an Event is set at 12:00 pm and the randomizeStart is set as -300 , the a client device can choose a number between [0,300]. 300 means five minutes, so if a client chooses -120 , then the Event will begin at 11:58 pm. The negative sign means a randomized time of an Event will be applied before an Event starts and the positive sign means the opposite [25].

\subsection{2 randomizeDuration}

Similar to the randomizeStart, randomizeDuration defines a time in seconds for when an Event ends. For example, if an Event is scheduled to end at 12:00 pm and the randomizeDuration is set as -120 , then the client device can choose a number from $[0,120]$. If a client chooses 60, the Event will end at 11:59 pm. A negative sign means an Event will end before the scheduled time and a positive sign means the opposite [25]. 


\subsection{3 opModEnergize}

The opModeEnergize attribute defines if the DER client connects or disconnects to the power source or electric grid. This attribute is a Boolean condition. 0 is "disconnected" and 1 is "connected" [25].

\subsection{4 opModFixedW}

To set Active Power Mode, two DER attributes are available: opModFixedW and opModTargetW. opModeFixedW determines a set point in percentage of maximum power. The opModTargetW determines a set point in Watts. However, opModTargetW is more suitable for aggregators, not for DER, so opModFixedW is selected to set the power level of the smart inverter in this research [25].

\subsubsection{DateTimeInterval}

The DateTimeInverval attribute is used to set the time interval of a DERControl event for a DER client. This attribute counts time in seconds. The time interval of each event shall be no longer than 86400 seconds (1 day) [25].

\subsection{6 opModVoltVar}

The opModVoltVar attribute defines a curve control-based static Volt-VAr function. The Volt-VAr curve is created by an array with multiple pairs of points. The pairs are interpolated into a linear function with hysteresis. The $\mathrm{x}$ value of each pair of points is a 
percentage voltage value refered to the reference voltage and the y value is a signed percentage value $(-100,100)[25]$.

\subsection{7 opModFreqWatt}

The opModFreqWatt attribute defines a curve controlled function. The Freq-Watt curve is created by an array with multiple pairs of points. The pairs are interpolated into a linear function with hysteresis [25]. The $\mathrm{x}$ value of each pair of points is a frequency value in $\mathrm{Hz}$ and the y value is a corresponding active power output value [25].

\subsection{DER Program Overview}

Each DER client is managed by a single DERControl event that follows the DER control algorithm. The DER control flow chart is shown in Figure 4.1. Before the control event is exposed to the DER client, the first step for clients is to identify which Function Set Assignment (FSA) applies to them. A FSA is a collection of resources related to the specific device. Then a DER client needs to distinguish the particular DERProgram related to the FSA. The DERProgram is composed of four control links: Default DER Control link, Active DER Control list link, DER Control list link, and DER Curve control list link. After entering the specific DER link, the client needs to determine which controls it needs. After distinguishing the specific controls, the client needs to read the control information and expose the related control parameters to the specific device based on the information. 


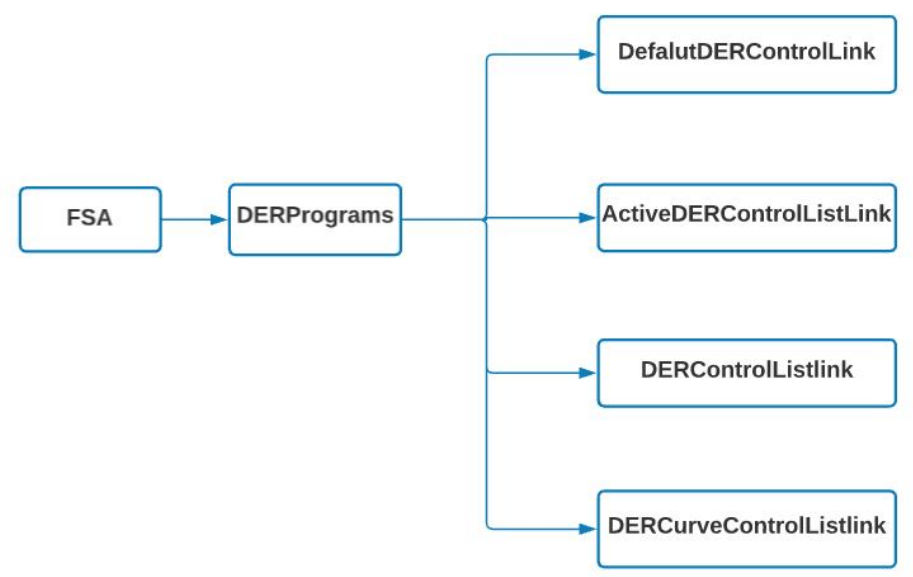

Figure 4.1: DER Program flow chart.

\subsection{DER Control Overview}

Before executing the control event, the client firstly determines if an active control event is coming. If there is no active event, then the client will use the default control to set the device. If the active event exists, then the client needs to identify the device category type to make sure if the event is for the device it controls. If the event is not for the device, then the client will ignore it. If the event is for the device, then the client needs to determine the status of the event. If the event is active, the client shall calculate the effective start time and specific end time. If the effective start time is in the past and the end time is in the future, then the client shall use the current time to set the control parameters by entering the control base. If the event is superseded, the client shall terminate the current event immediately and execute the new event. If the event is cancelled, the client shall determine if the cancellation event is applied to them and cancel the event instantly if possible. If the event is scheduled, the client need to keep the event until the event becomes active [25]. The flow chart of DER 
control is shown in Figure 4.2.

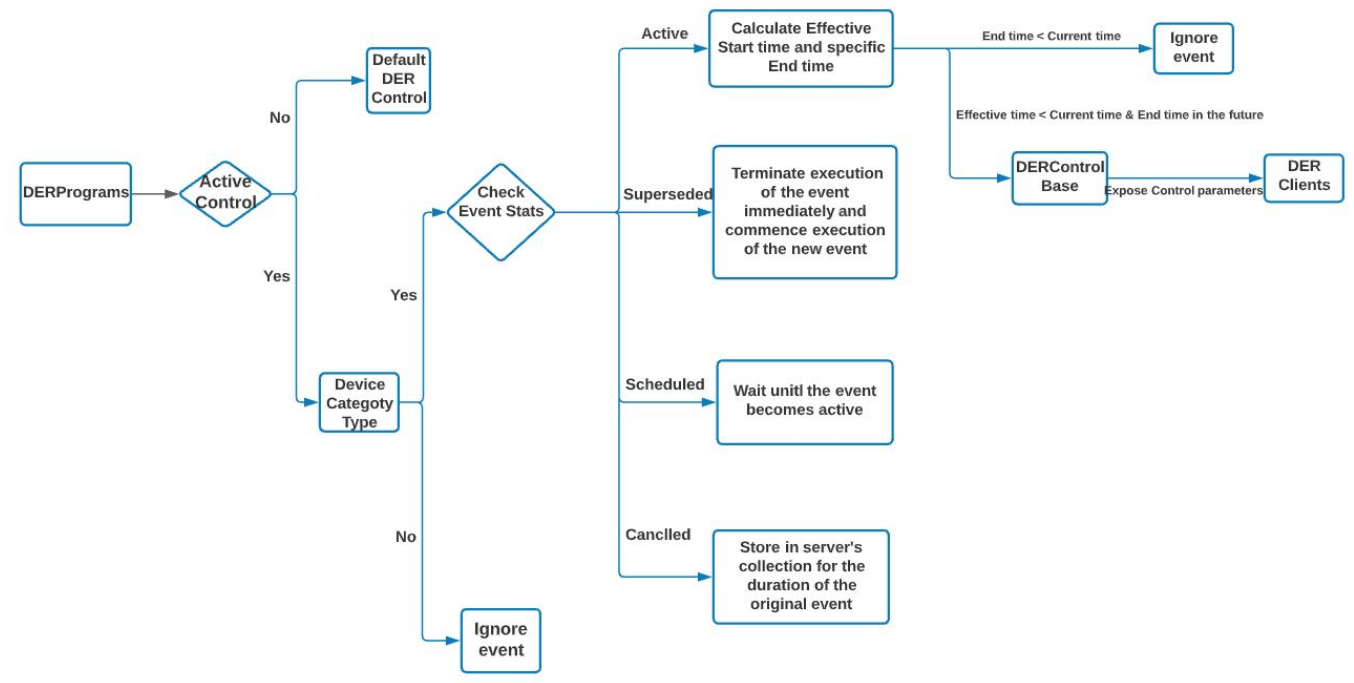

Figure 4.2: DER Control flow chart.

\subsection{DER Curve Overview}

Some functions of the distributed device, such as Volt-VAr and Frequency-Watt, are curvebased control function. For curve control events, the client need to firstly distinguish the curve type of the event and then set the relevant control parameters of the curve. To determine the shape of the curve, the client needs to set points on the curve using the curve data library.

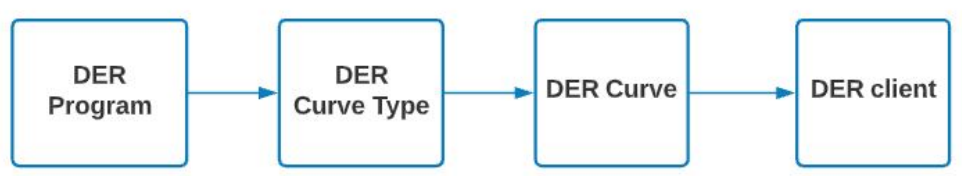

Figure 4.3: DER curve flow chart. 


\subsection{Testing Program of DER-Inverter}

The DER program developed in this research is mainly focused on executing the DERControl event to test the inverter. This DER program consists of three parts. The first part is trying to test if the inverter is capable of consuming or supplying power at a specific time. The second part is trying to test if Volt-VAr control can be enabled and used when IEEE 2030.5 attributes are involved to control the inverter. The third part is similar to the second part except the control function is changed to Frequency-Watt control. To control the inverter using the DER program, all IEEE 2030.5 attributes are mapped to related SunSpec Modbus registers in the AXS port. To create a scheduled time for a smart inverter to energize and de-energize, the Chrono library is used to get current time to refer to the Coordinated Universal Time (UTC). According to CSIP [13], the SunSpec registers in DER Grid Support Functions associated with IEEE 2030.5 attributes are shown in Table 4.1.

\begin{tabular}{|c|c|c|c|c|}
\hline Using Purpose & $\begin{array}{c}\text { IEEE 2030.5 } \\
\text { attribute }\end{array}$ & $\begin{array}{c}\text { SunSpec } \\
\text { Block }\end{array}$ & SunSpec Register & $\begin{array}{c}\text { SunSpec Register } \\
\text { Description }\end{array}$ \\
\hline $\begin{array}{c}\text { Energize or } \\
\text { De-energize } \\
\text { inverter }\end{array}$ & opmodEnergize & 123 & Conn & $\begin{array}{c}\text { Connection } \\
\text { Control }\end{array}$ \\
\hline $\begin{array}{c}\text { Set maximum } \\
\text { real power level } \\
\text { of inverter }\end{array}$ & opmodFixedW & 123 & WMaxLimPct & $\begin{array}{c}\text { Set maximum } \\
\text { Power lelve }\end{array}$ \\
\hline $\begin{array}{c}\text { Create a user- } \\
\text { defined Volt-VAr } \\
\text { Curve }\end{array}$ & opmodVoltVAr & 126 & Static Volt-VAr & $\begin{array}{c}\text { Static Volt-VAr } \\
\text { array }\end{array}$ \\
\hline $\begin{array}{c}\text { Crteate a user- } \\
\text { defined Freq-Watt } \\
\text { Curve }\end{array}$ & opmodFreqWatt & 134 & Freq-Watt Crv & $\begin{array}{c}\text { Curve Based } \\
\text { Freq-Watt array }\end{array}$ \\
\hline
\end{tabular}

Table 4.1: Mapping IEEE 2030.5 to SunSpec register. 


\section{Testing procedure}

To test the grid-enabled GS8048A smart inverter, specific testing procedures are required. The testing procedures include charging the battery, setting the inverter, configuring the grid simulator, installing the package on Raspberry Pi, running the DER program, and interacting with the smart inverter and grid simulator.

\subsection{Check battery level}

Before setting the GS8048A smart inverter, checking the battery voltage is always necessary since the inverter does not work until the battery voltage is greater than or equal to 44 VDC. To measure the battery voltage initially, the MATE3s system display and controller is used. The battery voltage information is located on the top right corner of the home screen of the MATE3s as shown in Figure 5.1. The battery voltage shown in 5.1 is a percentage value, but it can be programmed to show a real voltage value.

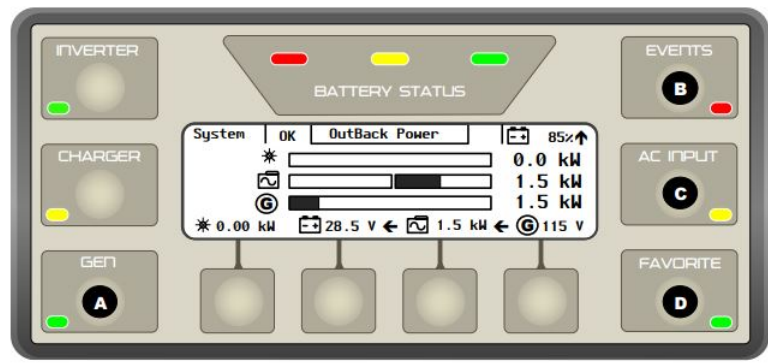

Figure 5.1: MATE3s home screen [27]. 
However, using the MATE3s to check battery voltage is only possible when the battery voltage is above $44 \mathrm{VDC}$. If the battery voltage below $44 \mathrm{VDC}$, a voltmeter has to be used to measure the voltage. This is done by measuring voltages between the hot and neutral terminals, which are connected below the smart inverter as shown in Figure 5.2. Note that a battery voltage measured using a voltmeter will be zero if the switch of the inverter is not in the $\boldsymbol{O}$ n position.

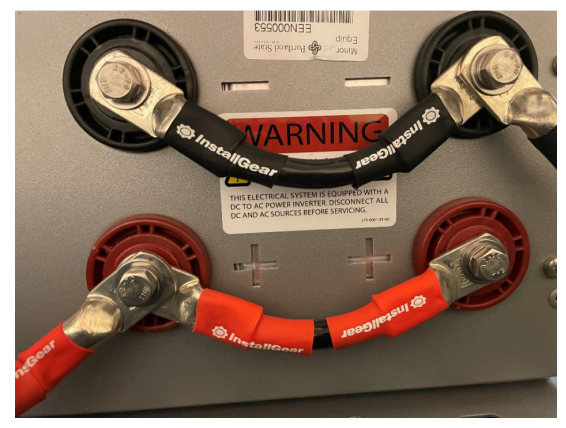

Figure 5.2: Hot and neutral line used to measure battery voltage.

If the battery voltage is lower than $44 \mathrm{VDC}$, the battery needs to be charged until the voltage level increases to 44 volts to initiate the GS8048A smart inverter. This process requires an external DC power supply to provide a constant current charge. The DC power supply used to do this is the PS305DM-DC Bench Power Supply from Dr. Meter, shown in Figure 5.3.

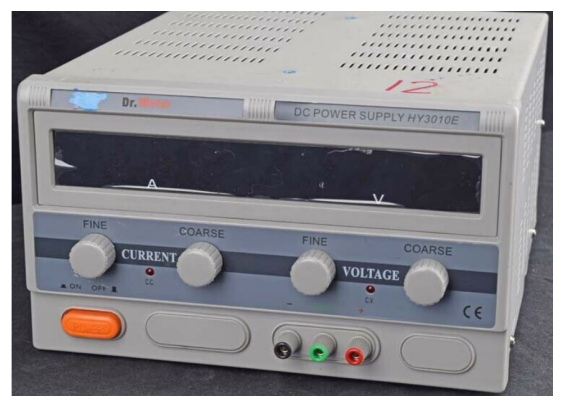

Figure 5.3: Dr.Meter Single-output Regulated Variable DC Power Supply. 
The maximum absorb voltage in the battery charge field of the GS8048A is 64 VDC [18].

The absorption voltage at $25^{\circ} \mathrm{C}$ ambient temperature for the Aquion ion battery is $66 \mathrm{VDC}$.

The average current into the battery is $10 \mathrm{~A}$ [21]. Since the ambient temperature of the inverter is usually around $25^{\circ} \mathrm{C}$, choosing a charge current of 10 Amps until the voltage increased to $44 \mathrm{VDC}$ is reasonable. The output constant current setting of the DC power supply can be found on pages 7 and 8 in section 4.2- Constant Current Settings in the PS305DM-DC Bench Power Supply user manual [28].

\subsection{Firmware update}

\subsubsection{Firmware checking}

In order to manipulate the inverter using the MATE3s and AXS port, the firmware versions of these devices should always support each other. The current firmware version of the GS8048A and the MATE3s can be found within the MATE3s. To check the firmware information, execute the following steps as ordered:

1. When the MATE3s illuminates after the inverter startes, click the hot key Lock on the MATE3s. At this time, a screen will shown as 5.4.

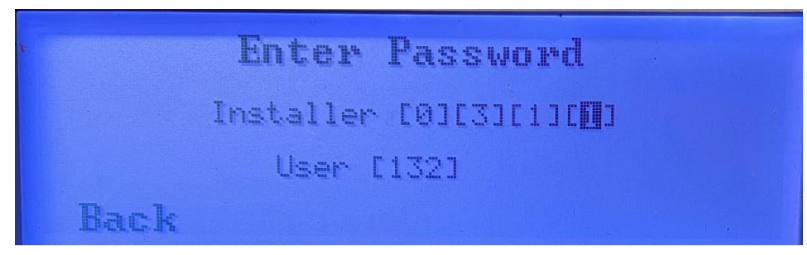

Figure 5.4: Password interface after click hot key lock.

2. The user number of the inverter is 132 and the installer password is 0311 . 
3. After entering the password, go to Setting $\rightarrow$ System $\rightarrow$ Firmware Versions.

4. All the firmware version of the devices that connected to GS8048A are shown here.

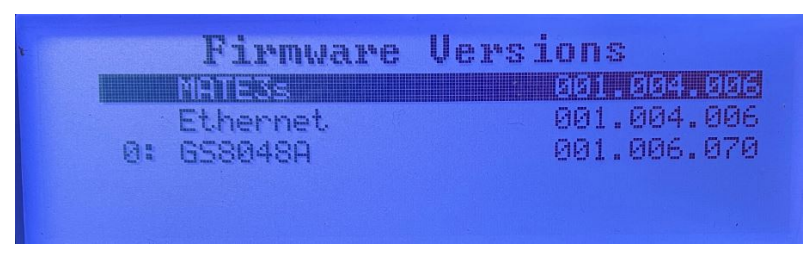

Figure 5.5: Firmware interface shown on MATE3s.

The user number 132 is to change the basic settings of the inverter. In this research, module GS8048A is modified in user 132. In case of change settings by mistake, user 141 is being used. Some functions are hidden in 141 such as module selection.

The MATE3s cannot read version information of the AXS port because MATE3s does not support communicate with the AXS port. Hence, the MATE3s cannot work at the same time as the AXS port. To check the firmware version of the AXS port, both approaches work as follow:

- open the read-me file that is stored in the SD card inserted in the AXS port

- read the OutBack Block information through using Modbus poll software

\subsubsection{Firmware Update}

The firmware update files of the MATE3s, AXS port, and GS8048A can all be found on the official website of Outback Power. Note that incompatible firmware versions between the MATE3s, AXS port, and GS8048A will lead to the MATE3s and AXS port being unable to control the GS8048A. Therefore, double check the firmware version of these devices. 


\subsubsection{AXS port update}

The firmware version of the AXS port can be updated using a micro SD card or using an FTP site with a micro SD card. However, directly using a micro SD card is easier for a user who firstly uses the AXS port. The specific procedures for updating the AXS port are well explained in the AXS port user manual on page 16-17 [20].

\subsubsection{MATE3s update}

The firmware update procedures are shown below:

1. Take the SD card from the MATE3s

2. Download the needed version of firmware from the official website

3. Then unzip the download folder

4. Open the download folder and keep the window open

5. Insert the SD card on the computer

6. Open the SD card folder and keep the window open

7. If any files existing in SD card, move the files to any folder you can memorize incase of unexpected changes

8. Format or erase the card before copying the firmware files
a) Back to the SD card window
b) Right-click the removeable drive icon and select format from the pop-up window.
c) Make sure the Files system is selected as FAT
d) Then press start

9. Copy all download files into the SD card folder 
10. Make sure have 5 files: WEBFILES, M3e.bin, M3Ethev3.bin, MATE3 (version number) Read Me.txt, screens.xml

11. Insert the SD card back to MATE3s

12. Power the MATE3s

13. Open MATE3s and enter the user number 141

14. Then go to Main Menu find Settngs $\rightarrow$ System $\rightarrow$ Clear Internal Date $\mathbf{L o g} \rightarrow$ Yes

15. Go back to Main Menu find Firmware update $\rightarrow$ MATE3 $\rightarrow$ Update

16. Do not unplug or reset until MATE3s Restarts.

17. Wait for two more minutes until the MATE3s can show information like battery voltage level

\subsubsection{GS8048A firmware update}

The procedures for updating the GS8048A is similar to that of the MATE3s.

1. Take the SD card from the MATE3s

2. Download the needed version of firmware from the official website

3. Then unzip the download folder

4. Open the download folder and keep the window open

5. Insert the SD card on the computer

6. Open the SD card folder and keep the window open

7. Copy all downlaod files into the SD card folder. (The existing files can be replaced)

8. Eject the SD card from computer

9. Insert the SD card back to MATE3s.

10. Power the MATE3s

11. Open MATE3s and change the user number to 141.

12. Go to Main Menu 


\section{Click Firmware update $\rightarrow$ GS Inverter}

14. The newer version firmware should dispaly

\section{Clikc Update}

16. Wait until the screen on the MATE3s shows "All Inverters Update"

\subsection{Setting the Grid Simulator}

The NHR 9410-12 grid simulator is used in this testing system to test the functions of the grid-enabled GS8048A inverter through control of grid frequency and voltage. Before interacting with the GS8048A, the configuration of the Grid simulator has to be changed and the monitor program has to be ready to observe the voltage, current, real power, complex power, and frequency. Since the grid simulator cannot directly connect with the inverter, a rack was created to provide a power interface, shown in Figure 5.6

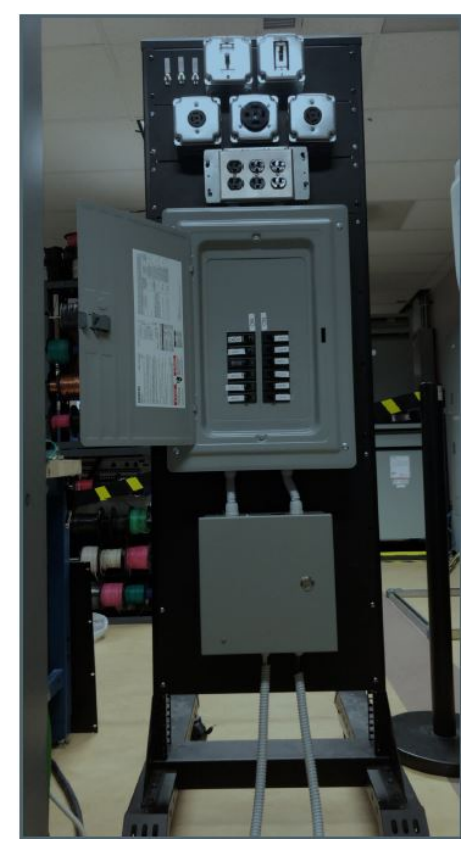

Figure 5.6: This rack provides a power interface between the GS8048A smart inverter and the NHR 9410-12 Grid Simulator. 


\subsubsection{Change configuration of grid simulator NHR 9410}

The Grid simulator NHR 9410 supports several different voltage configurations. To test grid-enabled smart inverters, the Grid-Simulator shall be adjusted to a specific configuration, 120/240 VAC.

1. Check the state of the grid simulator and make sure it is powered off. (As shown in Figure 5.7: turn the circuit breaker to the right to power off the grid simulator.)

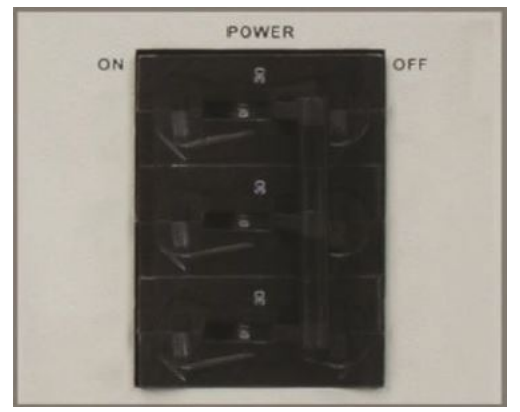

Figure 5.7: Circuit breaker of NHR 9410-12 smart grid simulator.

2. Configuring grid simulator to $120 / 240$ VAC split phase on the rack:

a) Turn on circuit breakers (the switch on the right) labeled as $240 \mathrm{~V}$ as shown in Figure 5.8.

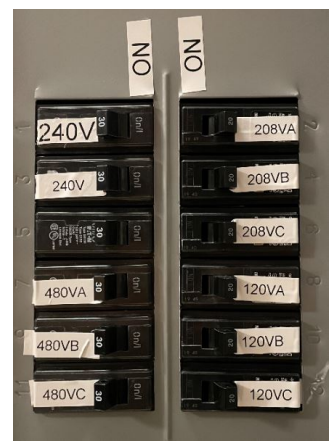

Figure 5.8: Circuit breakers of different output voltages. 
b) Change neutral switching mode into mode 5: One 2-phase AC and One AC

c) Turn off S-1, S-2, S-3, and S-4 neutral switches as shown below

d) Turn on S-5 neutral switch as shown below

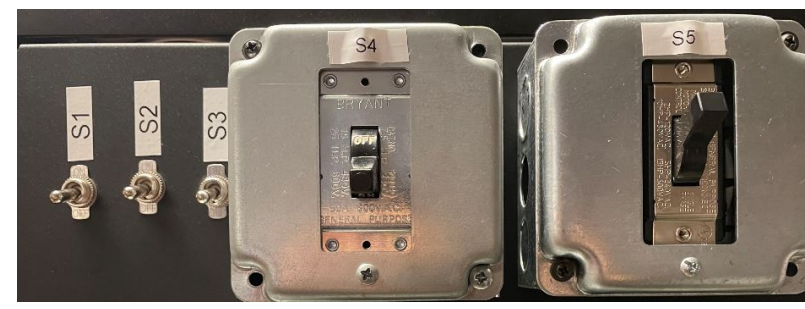

Figure 5.9: Neutral switch of different output voltages.

\subsubsection{Change settings of grid simulator using the software NHR 9400 panel}

a) Open the NHR 9400 Panel user interface on power lab computer PWRLAB04.ece.pdx.edu.

The NHR 9400 Panel interface is shown in Figure 5.10

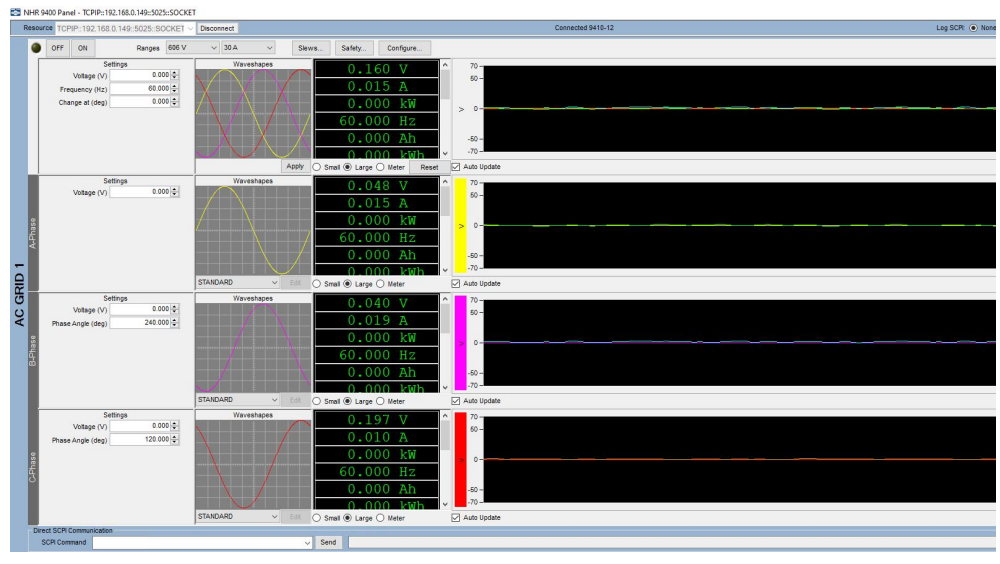

Figure 5.10: NHR 9410 grid simulator user interface software.

b) Verify the IP address of the NHR 9400 grid simulator on the top left corner $(192.168 .0 .149)$ 
c) Click "Connect" next to the IP address

d) Click "Configure" on the top right corner

e) A window will pomp out, Click "Yes" to change the configuration

f) A window called "Configure Hardware" will be displayed, as shown in Figure

\subsection{1}

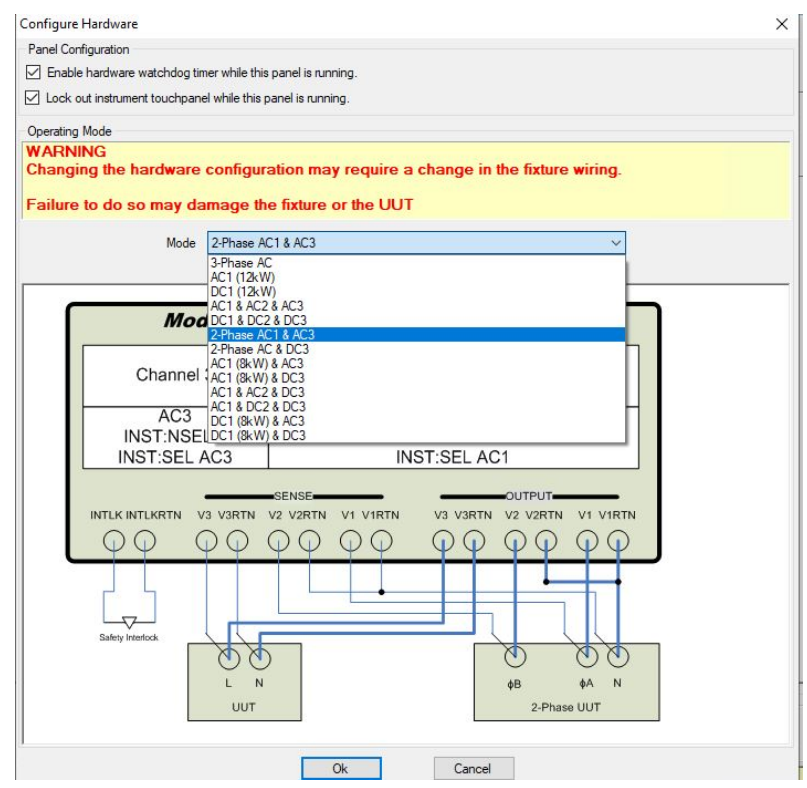

Figure 5.11: Change NHR grid simulator wire configuration setting.

g) Change the "Mode" to 2-Phase AC1 and AC3

h) Click "Ok" to finish setting split phase for inverter

i) Go back to home interface

j) Change the voltage value from top to the bottom in AC GRID 1 as shown in Figure 5.10

i. $240 \mathrm{~V}$ - The first voltage value (split phase value) 
ii. $120 \mathrm{~V}$ - The second voltage value (A-phase)

iii. $120 \mathrm{~V}$ - The third voltage value (B-phase)

3. Click "ON" to enable grid simulator. (Note: This step SHALL take action after connect the power trip of the inverter to the rack)

\subsection{Setting the GS8048A smart inverter}

The inverter is unable to undergo any testing if it is not in the correct operating mode and the connected battery voltage is low. Therefore, some settings must be changed to ensure the inverter is ready for testing.

\subsubsection{Turn on the inverter}

1. Put on insulating gloves

2. Connect power trip of inverter to the rack as shown in Figure 5.6

3. Turn the red switch on as shown in Figure 5.12

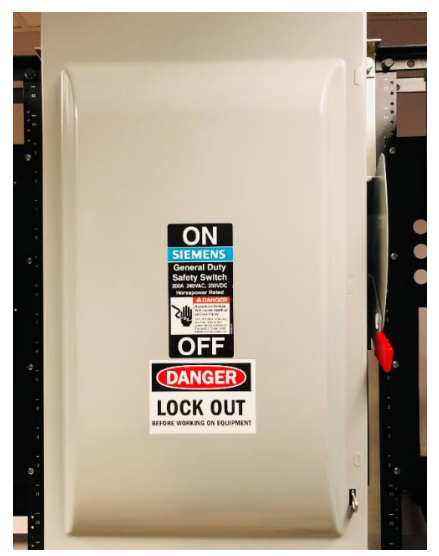

Figure 5.12: Power switch to turn on inverter. 


\subsubsection{Enable module GS8048A and GS4048A}

As mentioned before, the smart inverter used in this research has three modes. Mode 1: uses only one 4 kVA module (GS4048). Mode 2: uses two 4 kVA modules (GS8048) simultaneously, 0 - $8 \mathrm{kVA}$. Mode 3: uses two modules, but sequentially, firstly using one module for 0-4 kVA and then two modules for 4 kVA- 8 kVA (GS8048). The third module is more efficient but there is a switch-over period that affects performance. In this research, the mode 2 is used for testing the function of the inverter. The DC input power of GS8048A is $9.5 \mathrm{kVA}$ and output power is $8 \mathrm{kVA}$. Either mode mentioned above can be selected using MATE3s. To select the GS8048, the following steps should be executed as ordered:

1. If the GS8048A is working without any interruptions, then there is no need to enter the user number and installer password.

2. If the GS8048A is interrupted when it is normally working, then user has to enter the user number and installer password.

3. After entering the password, go to Setting $\rightarrow$ Inverter $\rightarrow$ Module select $\rightarrow$ GS8048A.

4. Select module GS8048A.

5. The mode that is currently selected will be shown in Figure 5.13.

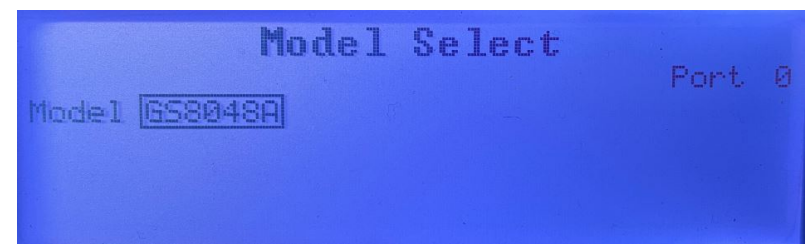

Figure 5.13: GS8048A is selected as the default mode. 


\subsubsection{Enable Grid-Tied Mode}

The Grid Tied mode enables the smart inverter to interact with the electric grid. In other words, the inverter can export power back to electric grid when excess power is available in the battery. However, in order to export power to the utility, the inverter has to use the operation of Offset. This operation is not a programmable function. It is an automatic operation that will export energy from the battery to compensate for the power when the load demand is higher than the power from the AC source. To enable the grid-tied mode, operate the MATE3s as followings:

1. If the GS8048A is working without any interruption, then there is no need to enter the user number and installer password.

2. If the GS8048A is interrupted when it normally working, then the user has to enter the user number and installer password.

3. After entering the password if needed, go to Setting $\rightarrow$ Inverter $\rightarrow$ Grid AC input Mode and Limits $\rightarrow$ select the Grid Tied input mode.

4. After finish setting, the result should be the same as Figure 5.14

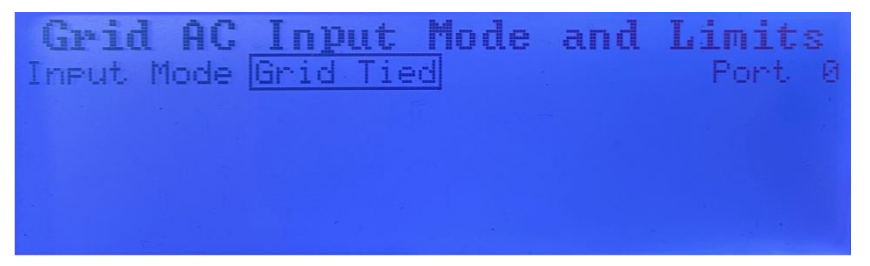

Figure 5.14: Grid Tied mode is selected as the operation mode.

To enable the Offset operation, the following steps should be executed as ordered: 
1. Back to Main Menu of MATE3s

2. Go to Setting $\rightarrow$ Inverter $\rightarrow$ Grid-Tie Sell

3. Inside of Grid-Tie Sell, the user can enable the offset operation, as shown in Figure 5.15

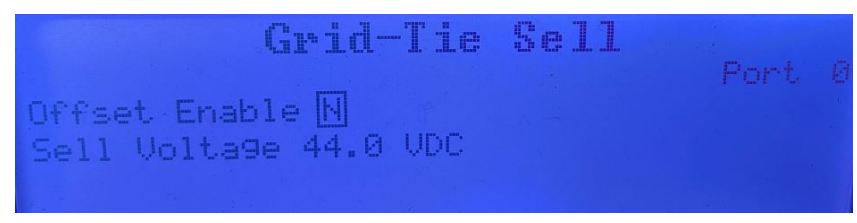

Figure 5.15: Offset operation enabled to export power from inverter.

\subsubsection{Change Relay Operation Time}

A time delay exists before the inverter exports power to the utility grid. In this time duration, the inverter will not connect to the grid and export power. This time setting is controlled by the function Re-Connect Delay Timer. The time is default-set to five minutes, but it is adjustable [18]. To change the relay time, the following steps should be executed in the MATE3s as ordered:

1. Back to Main Menu

2. Go to Settings $\rightarrow$ Inverter $\rightarrow$ Grid Interface Protection $\rightarrow$ Grid Support $\rightarrow$ Reconnect Parameters

3. In Reconnect Parameters, the Reconnect Delay can be changed, in seconds, as shown in Figure 5.16 


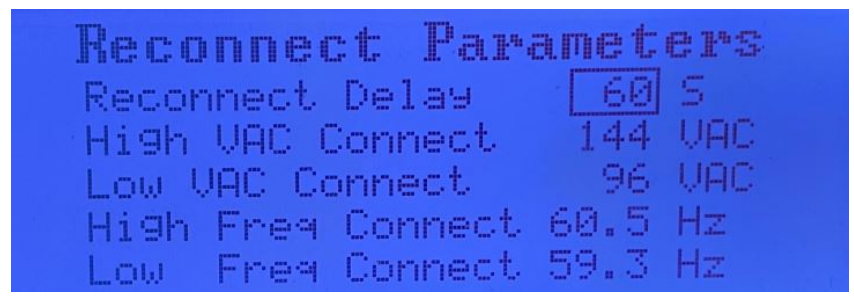

Figure 5.16: Time delay setting before inverter export power.

\subsubsection{Selling power to grid}

To test if GS8048A can export power from the inverter, some settings should be changed using the MATE3s in advance before exporting power from the inverter.

1. Go back to the main menu

2. GO to Inverter $\rightarrow$ Grid-Tie Sell $\rightarrow$ Enable the offset operation

3. Change the Sell Voltage value higher than current battery voltage.

4. Click hot key Inverter on MATE3s

5. Turn on the Mode from Off to On

6. Go back to home screen of the MATE3s

7. Oberving output power level of GS8048A.

\subsection{Identify IP address of AXS port}

Before modifying the SunSpec registers to control the GS8048A, the first step is to identify the IP address of the AXS port. To find the AXS port IP address, users first need to know 
the Media Access Control (MAC) address of the AXS port. The MAC address is a unique identifier used to distinguish this device in a network. The MAC address of the AXS port is labeled on the interface of the device. After identifying the MAC address of the AXS port, users can find the IP address that was randomly assigned by the router on the IP address interface of the router. Since the MAC address and the IP address are both shown on the IP address interface of the router, users determine the IP address of the AXS port directly. In Figure 5.17, the name "Unknown" with the MAC address 00:90:EA:E0:87:6D represents the AXS port.

\begin{tabular}{c|c|c|c} 
ID & Name & IP Address & MAC Address \\
\hline 1 & DESKTOP-GHOSMTS & 192.168 .0 .100 & $34:$ E8:94:F0:43:25 \\
\hline 2 & Unknown & 192.168 .0 .102 & $00: 90:$ EA:E0:87:6D \\
\hline 3 & pi3 & 192.168 .0 .101 & B8:27:EB:95:82:3B \\
\hline
\end{tabular}

Figure 5.17: TP-Link 6A92 LAN router interface.

\subsection{Charging Battery}

To keep the AHI battery healthy, the voltage level of the battery must always be maintained between 30 VDC and 59 VDC. If the voltage is outside of this safety voltage range for a while, chemical damage to the battery occurs. As mentioned before, the voltage reference points for the AHI battery should be set as shown in Table 3.1:

To set these reference voltage points and related time points, operate the MATE3s as following procedures: 
1. Back to Main Menu

2. Go to Settings $\rightarrow$ Inverter $\rightarrow$ Battery Charger

3. Change the voltage reference points and time as shown in 5.18

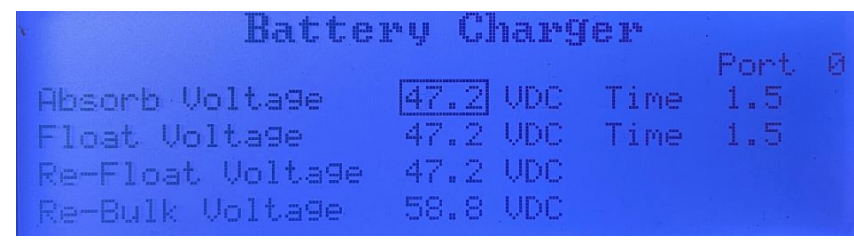

Figure 5.18: Voltage reference point and time for battery charging.

4. Bulk charge the inverter.

a) Click the hot key CHARGER on the MATE3s

b) Click the Bulk Charge after click on hot key CHARGER

c) Click Start Bulk after enter the Bulk charge

d) Click hot key Up on the MATE3s to go back the CHARGER interface

e) Click Charger Mode

f) Enable the charger control On

5. Wait until the battery finishes all charging stages

\subsection{Remote Development of the DER program on the Raspberry Pi}

To make sure the DER program can run on the Raspberry $\mathrm{Pi}$, and that the Pi can be remotely controlled, some configurations and documents have to be set and install on the Pi. The specific configure information and installation procedures of the $\mathrm{Pi}$ are shown in the 
Raspberry Pi tutorial on GitHub of Portland State University Power Lab. ${ }^{1}$ The following procedures are a general guide for users to understand how to configure a Raspberry Pi and remotely develop the DER program on a computer and then run it on Raspberry Pi.

1. Configuring the Raspberry Pi

a) Headless Setup - Remote control Raspberry Pi through SSH

b) Increase Swap memory - Increase RAM memory space

c) Dependency Installation - Develop and debug DER program

- Installing Cmake file

- Installing Boost file

- Installing XercesC file

d) Clone EGoT-DCS file on Raspbery Pi

e) Download the CLion Integrated Development Environment (IDE)

2. Interacting with the Raspberry Pi via computer ${ }^{2}$.

a) Download the CLion IDE

b) Clone the ssh-dev repository to the desired directory of the desktop environment

c) Clone the ssh-dev repository to the raspberry pi

d) Open CLion IDE and navigate to the ssh-dev repository

\footnotetext{
${ }^{1}$ https://github.com/PortlandStatePowerLab/raspberrypi-tutorial

${ }^{2}$ https://github.com/PortlandStatePowerLab/raspberrypi-tutorial
} 
e) Add a toolchain on the CLion IDE to create a host name for raspberry Pi and install Cmake on the local computer to interact with the Pi

f) Open the DER program file created on the Raspberry Pi on the computer and upload the DER program.

g) Debug and run the program

\subsection{Measuring output parameters from inverter}

To measure the output power, voltage, and current from the inverter, external measurement tools have to be used. In this testing system, the PA 4000 power analyzer is used for measuring power flow between the GS8048A inverter and the NHR 9410-12 Grid Simulator. To collect the power flow data, configure the PA 4000 as follows:

1. Choose one measurement channel to be used on the PA 4000 . The PA 4000 has four measurement channels. Each channel has the same voltage and current ratings.

2. Connect the PA 4000 to the junction box using test lead cables. Test leads must be rated 32 amps.

3. Turn on the PA 4000 power analyzer

4. Change the configuration of the PA 4000 into "One-phase, Three-wires" (1p3w)

5. Collect measurement values

- Collect measurement values on PA 4000 front panel

a) Press soft key MENU on PA 4000 front panel

b) Click Measurement on the interface 
c) Choose measurement values such as $V_{r m s}, A_{r m s}$, Watt, PF, etc

d) Go back to main manu to observe the changes of measurement values

e) Plug a USB drive into USB port on PA 4000

f) Observe the LED light next to the USB port. When it stop flushing, unplug the USB drive

g) The data are stored as a CSV file

- Read measurement values using the PA 4000 software-PWRVIEW [29]

a) Connect the power analyzer to the computer using an Ethernet cable

b) Open the PWRVIEW software on the desktop of computer

PWRLAB04.ece.pdx.edu

c) Make sure the IP address of the PA 4000 is configured in Network Setup

d) Press Add button to detect and identify the PA 4000 power analyzer

e) Click Connect button to connect the PA 4000 with the computer

f) Under Setup tab, choose the channel that the PA 4000 is connect to the power rack.

g) Choose $1 \mathrm{p} 3 \mathrm{w}$ next to the channel label

h) Choose $V_{r m s}, A_{r m s}$, Watt, PF in the Measurement Selection section.

i) Click Start or Stop in the Measurement tab to begin or stop measurement

j) Click Result to view the collected data files.

k) Choose the data file to store on the local computer 


\subsection{Checking SunSpec Registers Status}

The functions of the smart inverter are controlled using SunSpec registers. In order to check the SunSpec register status in the AXS port, multiple methods can be used. The AXS Port is the device that maps the internal register addresses of the inverter to the SunSpec Modbus registers. Using Modbus Poll software is the first method. Using the outback shall program created by the Outback power company is the second method. Running the DER program for testing the inverter is the third method. However, no matter which method is conduced, users must know the IP address of the AXS port and link it to the related software.

\subsubsection{Using modbus poll software}

To check the register status using Modbus Poll, conduct the following procedures in order:

1. Open Modbus Poll software on the desktop of the power lab computer: PWRLAB06.ece.pdx.edu

2. Click Connection on the header

3. Choose Modbus TCP/IP as default connection

4. Enter IP-address of the AXS port

5. Choose IPv4 as internet protocol version

6. Click File $\rightarrow$ New, then a window will pop up.

7. Right click the data address on the right column and choose "Read/write Definition"

8. Find the particular SunSpec Modbus register model address that begins with 40011 . Each block is clearly labeled with a number at the beginning and has a specific length. The block information is contained in the SunSpec Information Model Reference. 
9. Change the Quality to 125 . This is the maximum number of registers that can be shown in a single window. Multiple windows are needed if hundreds of registers are being checked.

10. In the view section, choose Fit to Quality

\section{Click OK}

12. The Alias of each register can be changed refer to the specific version of SunSpec Information Model Reference

13. The SunSpec block such as "Immediate Control 123" shown in the Modbus poll will be same as shown in Table 5.1

\begin{tabular}{|c|c|c|}
\hline Register address & Alias & Register status \\
\hline 40736 & ID & 123 \\
\hline 40737 & length & 24 \\
\hline 40739 & Conn_WinTms & -1 \\
\hline 40740 & Conn_RvrtTms & -1 \\
\hline 40741 & Conn & 1 \\
\hline 40742 & WMaxLimPct & 50 \\
\hline 40743 & WMaxLimPct_WinTms & -1 \\
\hline 40744 & WMaxLimPct_RvrtTms & -1 \\
\hline 40745 & WMaxLimPct_RmpTms & -1 \\
\hline 40746 & OutPFSet & 100 \\
\hline 40747 & OutPFSet_WinTms & -1 \\
\hline 40748 & OutPFSet_RvrtTms & -1 \\
\hline 40749 & OutPFSet_RmpTms & -1 \\
\hline 40750 & OutPFSet_Ena & 0 \\
\hline 40751 & VArWMaxPct & -32768 \\
\hline 40752 & VArMaxPct & -32768 \\
\hline 40753 & VArAvalPct & -32768 \\
\hline 40754 & VArPct_WinTms & -1 \\
\hline 40755 & VArPct_RvrtTms & -1 \\
\hline 40756 & VArPct_RmpTms & -1 \\
\hline 40757 & VArPct_Mod & -1 \\
\hline 40758 & VArPct_Ena & -1 \\
\hline 40759 & WMaxLimPct_SF & 0 \\
\hline 40760 & OutPFSet_SF & -2 \\
\hline 40761 & VArPct_SF & 0 \\
\hline
\end{tabular}

Table 5.1: SunSpe registers as shown in Modbus Poll. 
14. Check the register Conn to verify if the inverter is energize or not

15. Check the register WMaxLimPct to verify maximum power output level defined by the users.

The models that contain the registers used for testing inverter functions are shown in the file labled as Modbus PSU on the desktop of the PWRLAB06.ece.pdx.edu computer.

\subsubsection{Using Outback Shell program}

The Outback shell program uses created by the Outback Power Company, but the company no longer maintains it, which means the most recent SunSpec blocks cannot be read in the AXS port. According to the Outback Power technical support team, the SunSpec registers are not all correct to use. Many SunSpec registers have not implemented.

1. Open the file OutbackShell SDK file on the Desktop of the PSU power lab computerPWRLAB06.ece.pdx.edu

2. Execute the outback.shell application in the OutbackShell SDK file.

3. Click 'h' to get more commands to operate the shell program as shown in Figure 5.19

4. Identify the AXS port IP address

5. Click 'c' to enter the IP address of the AXS port

6. Then all SunSpec blocks in the AXS port will appear once the Outback Shell program is connected to the AXS port

7. Click 'r' to identify the offset number of SunSpec blocks and registers

8. Click 'w' write the values into specific SunSpec blocks or registers 


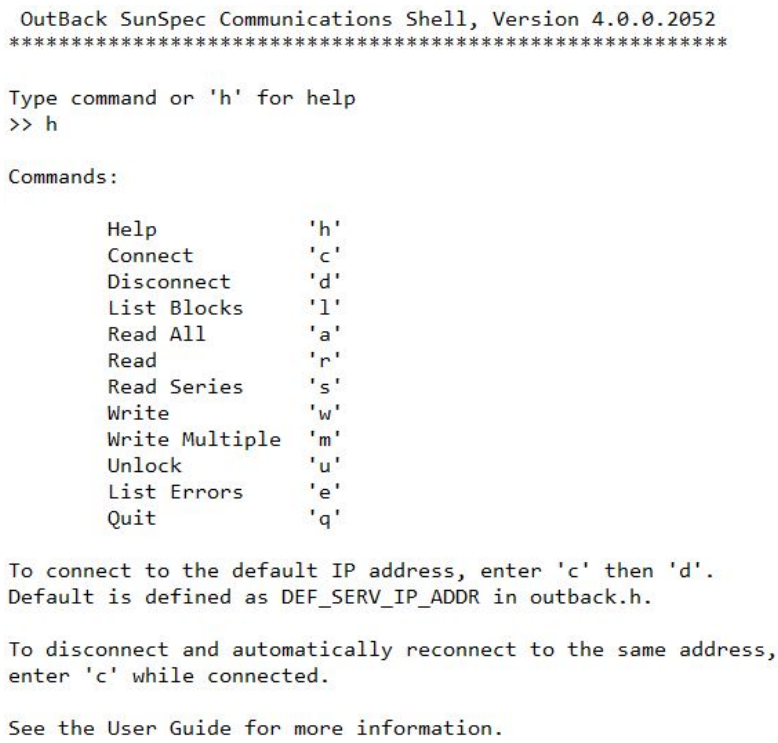

Figure 5.19: Outback shell program designed for AXS port.

\subsubsection{Running DER program}

1. Connect Raspberry Pi to a monitor or ssh into Raspberry Pi

2. Go to Energy Grid of Things (EGoT)-DER $\rightarrow$ EGoT-DCS $\rightarrow$ src

3. Figure out the AXS port IP address. When the Pi does not connect to the AXS port, connection errors will appear and simulation model will be created.

4. Enter the IP address assigned by the router in the DER program

5. Compile the main.cpp file in the src folder under the path EGoT-DER/EGoT-DCS/src. (cmake -build build/)

6. Build the main.cpp file (./build/src/app). Then, all SunSpec blocks and register information will be shown. 


\section{Results \& Analysis}

\subsection{Verify The Values Written Into The SunSpec Registers}

To make sure the DER program can detect the SunSpec blocks and the registers in the block in the AXS port, the first thing to do is checking if the number of blocks and registers in the AXS port match with the SunSpec model reference. If the number of SunSpec blocks and their length does not match with the reference, then the DER program will be terminated as it is running and will not read all the SunSpec blocks and registers in the AXS port. All the SunSpec blocks and registers in the AXS port are created based on the specific version of the SunSpec model reference and type noted in the XML files for users to read and modify.

In order to check the number of SunSpec blocks and registers in the AXS port, the Outback shell program is used. According to the Outback Power technical support group, the people who created the SunSpec models for the AXS port have left the company. Therefore, Outback is not sure which version of the SunSpec model that the AXS port applies. The SunSpec model references that were created in 2015 and later can be found on the SunSpec organization website. Through comparing the SunSpec models in the AXS port and the reference, some differences were found. As shown in Figure 6.1, block 64110 "Outback AXS devices" has 420 registers, but the number of registers defined in the reference only have 282. Other SunSpec blocks such as 64120, 64115, 64116, and 64255 created in the 
AXS port are not defined in the SunSpec model references that were created in 2015 and later. ${ }^{3}$ Therefore, the programmer has to add the additional blocks and registers defined in the AXS port to the XML SunSpec model base for the DER program to use. To complete the XML SunSpec models for DER programs, the additional information of the SunSpec blocks in the AXS port can be found on the Outback Power company website. Once the XML SunSpec models are created and kept the same with the AXS port, the DER program is ready to run. But not all SunSpec registers in the AXS port allow the values to be passed. ${ }^{4}$

\begin{tabular}{|c|c|c|c|}
\hline \multicolumn{2}{|c|}{ SunSpec Device } & \multicolumn{2}{|c|}{ Detected! } \\
\hline$\cdots$ & $-\ldots$ & $-\cdots$ & -- \\
\hline Block & Address & DID & Offset \\
\hline$\ldots$ & 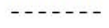 & $-\cdots$ & $-\ldots-$ \\
\hline$\theta$ & 40002 & 1 & 65 \\
\hline 1 & 40069 & 64110 & 420 \\
\hline 2 & 40491 & 64120 & 27 \\
\hline 3 & 40520 & 102 & 50 \\
\hline 4 & 40572 & 64255 & 56 \\
\hline 5 & 40630 & 120 & 26 \\
\hline 6 & 40658 & 121 & 30 \\
\hline 7 & 40690 & 122 & 44 \\
\hline 8 & 40736 & 123 & 24 \\
\hline 9 & 40762 & 124 & 24 \\
\hline 10 & 40788 & 126 & 64 \\
\hline 11 & 40854 & 129 & 60 \\
\hline 12 & 40916 & 130 & 60 \\
\hline 13 & 40978 & 132 & 64 \\
\hline 14 & 41044 & 134 & 68 \\
\hline 15 & 41114 & 135 & 60 \\
\hline 16 & 41176 & 136 & 60 \\
\hline 17 & 41238 & 137 & 60 \\
\hline 18 & 41300 & 138 & 60 \\
\hline 19 & 41362 & 139 & 60 \\
\hline 20 & 41424 & 140 & 60 \\
\hline 21 & 41486 & 145 & 8 \\
\hline 22 & 41496 & 64115 & 58 \\
\hline 23 & 41556 & 64116 & 89 \\
\hline 24 & 41647 & 65535 & 0 \\
\hline & & -1 & \\
\hline
\end{tabular}

Figure 6.1: SunSpec blocks and their length in AXS port.

\footnotetext{
${ }^{3}$ SunSpec Information Model Reference 09/28/2017 or later

${ }^{4}$ https://www.outbackpower.com/downloads/documents/system ${ }_{m}$ anagement/ax $s_{p}$ ort/ax $s_{a} p p_{n}$ ote.pdf
} 


\subsection{Energize and De-energize Testing}

In order to test the interoperability of a smart inverter, the first tests conducted in this research are energizing and de-energizing. As shown in Table 6.1, when the user-defined value 1 (energize) or 0 (de-energize) was transferred into the SunSpec register Conn after executing the DER program, the status of the register will be changed to "Connect" or "Disconnect".

\begin{tabular}{|c|c|c|c|c|}
\hline $\begin{array}{c}\text { IEEE 2030.5 } \\
\text { attribute }\end{array}$ & $\begin{array}{c}\text { Transfer } \\
\text { Value }\end{array}$ & $\begin{array}{c}\text { SunSpec } \\
\text { Register }\end{array}$ & $\begin{array}{c}\text { SunSpec } \\
\text { Register } \\
\text { status }\end{array}$ & $\begin{array}{c}\text { Connect } \\
\text { or } \\
\text { Disconnect }\end{array}$ \\
\hline op_mod_energize & WritePoint: 40740,1 & Conn & 1 & Connect \\
\hline op_mod_energize & WritePoint: 40740,0 & Conn & 0 & Disconnect \\
\hline
\end{tabular}

Table 6.1: Result of writing and transferring IEEE 2030.5 "opmodConnect" to SunSpec "Conn"

To make sure the user-defined value was indeed transferred into the SunSpec register Conn, the Outback Shell program was used. As shown in Table 6.2, the SunSpec register is "Connect" when the defined value 1 was transferred and Disconnect when $\mathbf{0}$ was transferred.

\begin{tabular}{|c|c|c|c|}
\hline Register Address & Block offset & Register Name & Value \\
\hline 40736 & 882 & IMMED_INVERTER_CONTROLS_DID & 123 \\
\hline 40737 & 883 & IMMED_INVERTER_CONTROLS_LEN & 24 \\
\hline 40738 & 884 & IMMED_INVERTER_CONTROLS_Conn_WinTms & Not implemented \\
\hline 40739 & 885 & IMMED_INVERTER_CONTROLS_Conn_RvrtTms & Not implemented \\
\hline 40740 & 886 & IMMED_INVERTER_CONTROLS_Conn & Connect \\
\hline 40740 & 886 & IMMED_INVERTER_CONTROLS_Conn & Disconnect \\
\hline
\end{tabular}

Table 6.2: Verify energize and de-energize numbers were transferred into SunSpec registers using OutBack Shell Program 
To verify the inverter will be energized after the value is transferred into the register,

SunSpec block 102 - Split Phase inverter is used. As shown in Table 6.3, the phase current and phase voltage value will be updated to the defined voltage and current value after the inverter is connected to the grid. Those updated values will remain as 0 once the inverter is de-energized.

\begin{tabular}{|c|c|c|c|}
\hline Register Address & Block offset & $\begin{array}{c}\text { SunSpec } \\
\text { Resiter name }\end{array}$ & Value \\
\hline 40520 & 304 & I_SunSpec_DID & 102 \\
\hline 40521 & 305 & I_SunSpec_Length & 50 \\
\hline 40522 & 306 & I_AC_Current & 24.30 A \\
\hline 40523 & 307 & I_AC_CurrentA & 12.54 A \\
\hline 40524 & 308 & I_AC_CurrentB & 11.69 A \\
\hline 40525 & 309 & I_AC_CurrentC & 0 A \\
\hline 40526 & 310 & I_AC_Current_SF & 0 \\
\hline 40527 & 311 & I_AC_VoltageAB & 244 V \\
\hline 40528 & 312 & I_AC_VoltageBC & Not implemented \\
\hline 40529 & 313 & I_AC_VoltageCA & Not implemented \\
\hline 40530 & 314 & I_AC_VoltageAN & 122 V \\
\hline 40531 & 315 & I_AC_VoltageBN & 122 V \\
\hline 40532 & 316 & I_AC_VoltageCN & Not implemented \\
\hline
\end{tabular}

Table 6.3: Verify Energize or De-energize of an inverter through observing the voltage and current changes

\subsubsection{Energize and De-energize Testing Analysis}

From above test results, the inverter is compliant with IEEE 2030.5 attribute opModEnergize. In conclusion, the inverter can provide a functionally correct response to the IEEE 2030.5 attibute related to energize or de-energize. 


\subsection{Maximum Export Power Testing}

SunSpec register "WMaxLimPct" determines the maximum power level of the inverter, which is the representative register that allows the values to be changed. If the value "80" can be transferred into the register, then the status of the register can be verified using the function "ReadBlock" and "PrintBlock" after running the DER program. If the value cannot be transferred into the register, then a reason such as "Illegal Data Value" will be shown to remind the user. If the value successfully transfers, then the grid operator can set parameters for the inverter by using the IEEE 2030.5 attributes and the attributes can be linked to the specific SunSpec register. The result of setting the maximum power of the inverter is summarized in Table 6.4.

\begin{tabular}{|c|c|c|c|c|}
\hline $\begin{array}{c}\text { IEEE 2030.5 } \\
\text { attribute }\end{array}$ & $\begin{array}{c}\text { Transfer Value } \\
(\boldsymbol{\%})\end{array}$ & SunSpec Register & $\begin{array}{c}\text { SunSepec Register } \\
\text { Status (\%) }\end{array}$ & $\begin{array}{c}\text { Maximum Export } \\
\text { Power }\end{array}$ \\
\hline op_mod_fixed_w & 80 & WMaxLimPct & 80 & No response \\
\hline op_mod_fixed_w & 6 & WMaxLimPct & 6 & No response \\
\hline op_mod_fixed_w & 5 & WMaxLimPct & Illegal Data Value & No response \\
\hline op_mod_fixed_w & 100 & WMaxLimPct & Illegal Data Value & No response \\
\hline
\end{tabular}

Table 6.4: Test result of verifying connection and setting maximum power of the inverter.

Any value greater or equal to $6 \%$ and lower or equal to $80 \%$ can be written into the register "WMaxLimPct". The value in SunSpec register "WMax" in SunSpec block 121 (Basic Settings) will vary as the value changes in the register "WMaxLimPct". The SunSpec register "WMax" will be used as the reference in frequency-Watt control to set the maximum power that the inverter can export or absorb in the later section. The maximum power that 
inverter can absorb or export is 7200 VA according to value shown in the "WMax" as the "WMaxLimPct" was set to $100 \%$. The scale factor of SunSpec register "WMax" is 1, which means the decimal point moves to the right by one position.

To double check if the inverter has been correctly written into the SunSpec register "WMaxLimPct", the Outback shell program is used. As shown in Figure 6.2, the value has been successfully passed into the SunSpec register "WMaxLimPct".

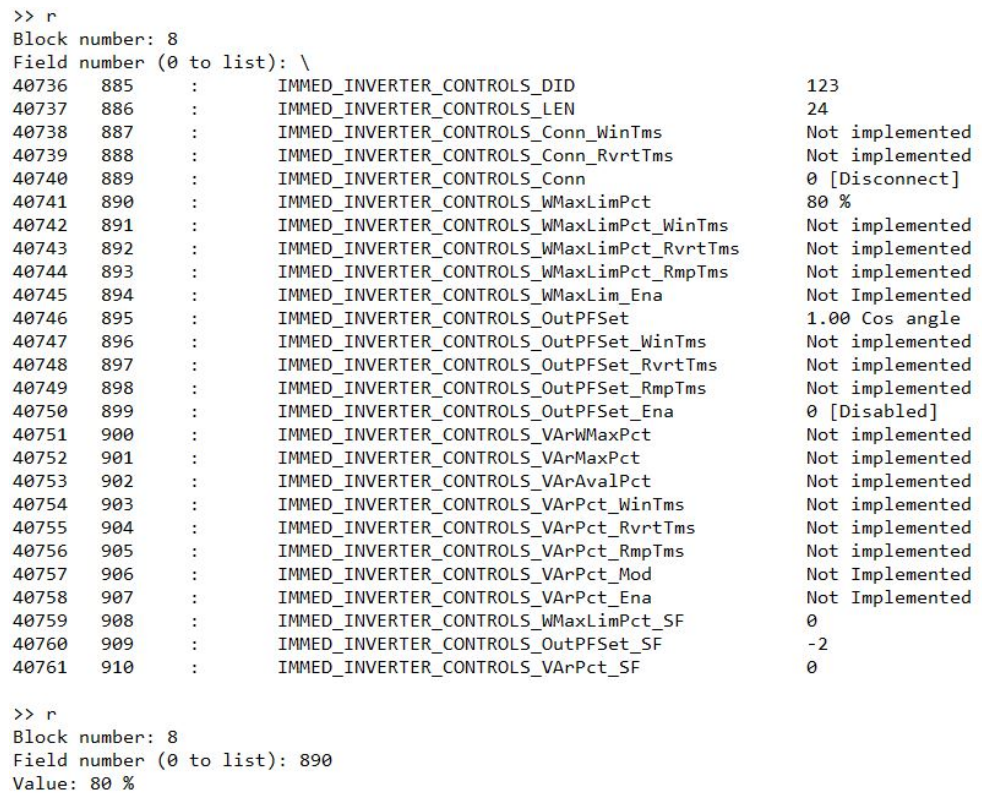

Figure 6.2: The maximum power value of the inverter had been successfully written into SunSpec resiter 'WMaxLimPct'.

\subsubsection{Maximum Export Power interoperability Testing Analysis}

Based on the above testing result, percentage value in the range of $[6,80]$ defined in IEEE 2030.5 attribute can be transferred in the SunSpec register WMaxLimPct, but the inverter will not use the SunSpec parameters to enable the function and export the maximum power 
as defined. Therefore, the testing system discovers that the GS8048A smart inverter is not fully compliant with IEEE 2030.5 opModFixedWatt attribute. In conclusion, the inverter does not provide a functionally correct response to the IEEE 2030.5 attribute.

\subsubsection{Maximum Power Export Testing Connected with Grid Simulator}

Even the inverter is not compliant with attribute opModFixedWatt, the testing system can perform the test of exporting maximum power of an inverter when the inverter is connected to a grid simulator. To enable the maximum export power function of the inverter, the voltage reference points must be used, which are Absorb voltage and Sell voltage. These two voltages must be set at the same value to enable the maximum power output function of an inverter. In order to export the maximum power, the Absorb voltage and Sell voltage must be set at the lowest value which is 44 VDC. As shown in the figure 6.3 , when the voltage reference points were set as the minimum value, the inverter will export the maximum power around $3.3 \mathrm{~kW}$.

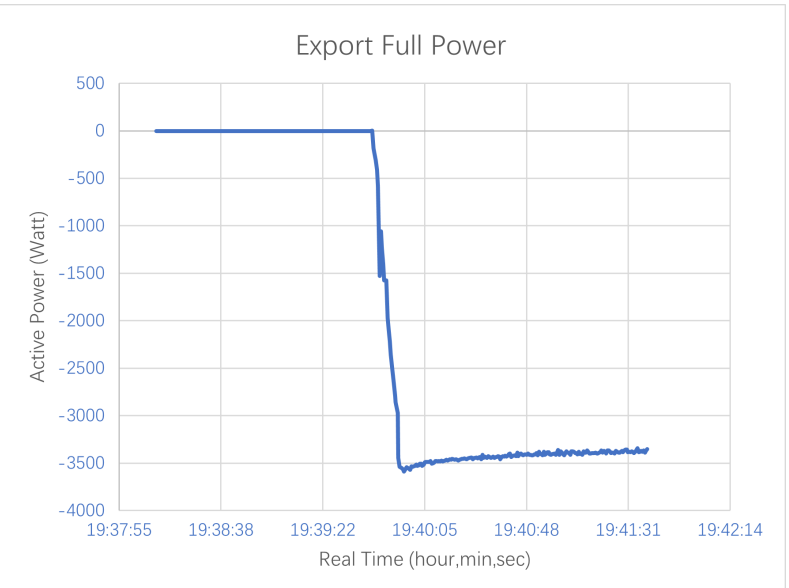

Figure 6.3: Exporting maximum power from inverter. 
The reason that active power is negative is because the power value was measured on the grid-simulator side, so the export power from the inverter means import power to the grid-simulator. The LED light on the grid simulator indicates the direction of power. As shown in Figure 6.4, the power flows into the grid-simulator. In Figure 6.5, the power flows out from the grid-simulator. "Sink" means power goes in, "source" means power goes out.

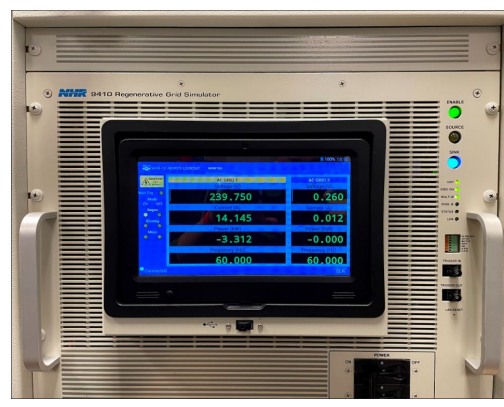

Figure 6.4: Grid simulator absorb power from load, blue LED light.

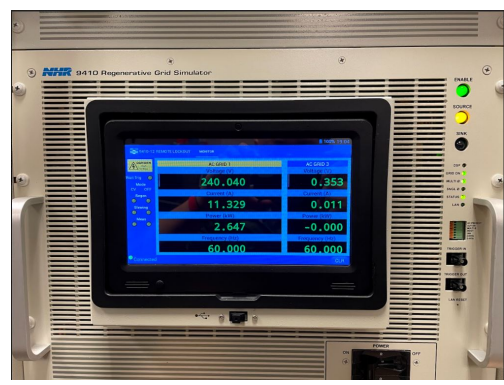

Figure 6.5: Grid simulator export power to load, yellow LED light.

To change the maximum power level from the inverter, simple calculation is required. For example, if the maximum battery voltage is $60 \mathrm{VDC}$ and the Absorb voltage and Sell voltage were set as $52 \operatorname{VDC}((60-44) / 2+44=52)$, then inverter will export half of maximum power around $1.65 \mathrm{~kW}$, as shown in Figure 6.6. 


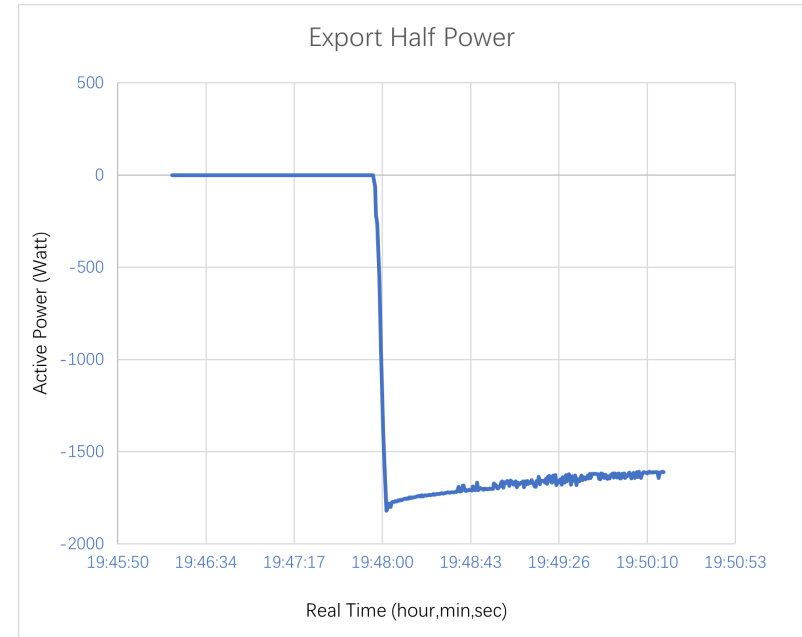

Figure 6.6: Exporting half power from inverter.

\subsubsection{Maximum Export Power Sub-component Testing Analysis}

Based on the above test result, the testing system has the ability to test the maximum export power from an inverter especially when the inverter is connected to a grid simulator. Also, the testing system shows it can perform different exported power levels testing for the inverter. 


\subsection{Volt-VAr function Testing}

The Volt-VAr function is an autonomous control function, which means the inverter will produce or consume reactive power autonomously when it senses a grid voltage that is lower or higher than the limit. When the grid AC voltage lower than the nominal volatge, the inverter will produce capacitive power. When the grid AC voltage is higher than the nominal voltage, the inverter will consume inductive power.

Since the Volt-VAr function is a curve-based control, it is necessary to choose points to construct the curve. To construct a Volt-VAr curve, at least four points shall be used, as shown in Figure 6.7. Point (V1,Q1) sets the maximum capative reactive power that inverter will provide. Points (V2,Q2) and (V3,Q3) set the dead-band for the inverter. When grid AC voltage in the dead-band, the inverter has no reaction to the grid voltage. The dead-band shown in Figure 6.7 the reactive power is 0 , but usually the reactive power is not 0 . It will be the minimum reactive power that inverter will produce. Point $(\mathrm{V} 4, \mathrm{Q} 4)$ sets the maximum inductive reactive power that inverter will consume.

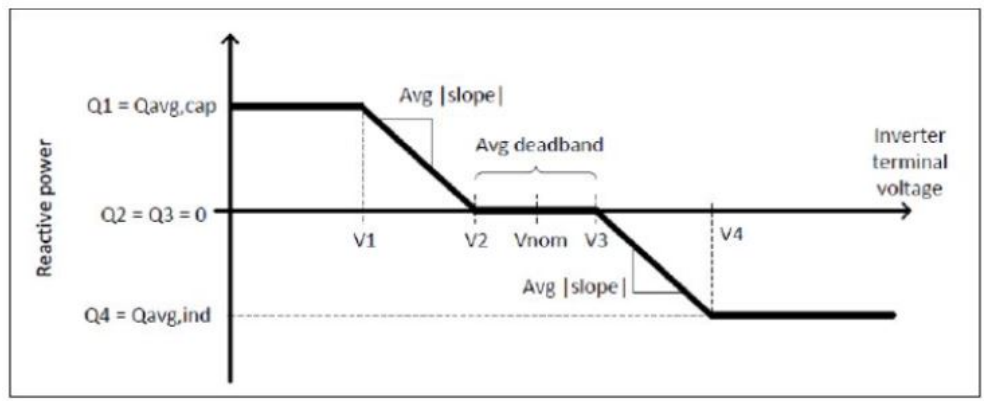

Figure 6.7: Standard Volt-VAr curve [12]. 


\subsubsection{Verification of Writing Volt-VAr Curve Points}

In the DER program, the $(V 1, Q 1)$ was set as $(41,460),(V 2, Q 2),(V 3, Q 3)$ were set as $(44,0),(52,0)$, and $(V 4, Q 4)$ was set as $(55,460)$. Since $\mathrm{x}$ value in each pair of points is a percentage value, $41.00 \%=240 \times 0.41=98.4 V A C, 44.00 \%=240 \times 0.44=105.6 \mathrm{VAC}$, $52.00 \%=240 \times 0.52=124.8 V A C, 55.00 \%=240 \times 0.55=132 V A C$. In SunSpec register, the default sign of Q4 is negative. Table 6.5 shows the process and the result of Volt-VAr curve points defined in IEEE 2030.5 attributes and transferred into the SunSpec registers after executing the DER program. Based on the curve created above, the expected Volt-VAr curve will like as shown in Figure 6.8.

\begin{tabular}{|c|c|c|c|}
\hline IEEE 2030.5 attribute & Transfer Value & $\begin{array}{c}\text { SunSpec } \\
\text { Register }\end{array}$ & $\begin{array}{c}\text { SunSpec Register } \\
\text { Status }\end{array}$ \\
\hline $\begin{array}{c}\text { opmodVoltVAr } \\
\text { CurveData vv[0].x=41 }\end{array}$ & WritePoint: 40802,4100 & V1 & 4100 \\
\hline $\begin{array}{c}\text { opmodVoltVAr } \\
\text { CurveData vv[0].y=460 }\end{array}$ & WritePoint: 40803,460 & VAr1 & 460 \\
\hline $\begin{array}{c}\text { opmodVoltVAr } \\
\text { CurveData vv[1].x=44 }\end{array}$ & WritePoint: 40804,4400 & V2 & 4400 \\
\hline $\begin{array}{c}\text { opmodVoltVAr } \\
\text { CurveData vv[1].y=0 }\end{array}$ & WritePoint: 40805,0 & VAr2 & 0 \\
\hline $\begin{array}{c}\text { opmodVoltVAr } \\
\text { CurveData vv[2].x=52 }\end{array}$ & WritePoint: 40806,5200 & V3 & 5200 \\
\hline $\begin{array}{c}\text { opmodVoltVAr } \\
\text { CurveData vv[2].y=0 }\end{array}$ & WritePoint: 40807,0 & VAr3 & 0 \\
\hline $\begin{array}{c}\text { opmodVoltVAr } \\
\text { CurveData vv[3].x=55 }\end{array}$ & WritePoint: 40808,5500 & V4 & 5500 \\
\hline $\begin{array}{c}\text { opmodVoltVAr } \\
\text { CurveData vv[3].y=460 }\end{array}$ & WritePoint: 40809,460 & VAr4 & 460 \\
\hline
\end{tabular}

Table 6.5: Using DER program to set curve points for Volt-VAr function. 


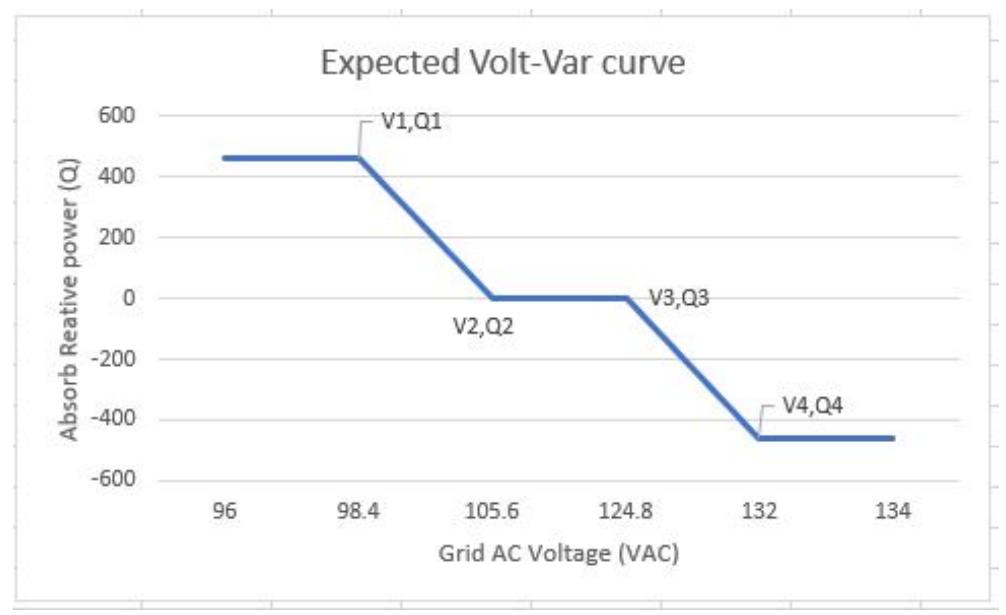

Figure 6.8: Expected Volt-VAr curve based on the user defined curve points.

To make sure if the Volt-VAr curve points are written into the AXS port, Outback Shell program is used. Table 6.6 summarized the test results of Volt-VAr curve points that are written into the SunSpec registers.

\begin{tabular}{|c|c|c|c|}
\hline Register Address & Block offset & Register Name & Value \\
\hline 40788 & 937 & STATIC_VOLT_VAR_DID & 126 \\
\hline 40789 & 938 & STATIC_VOLT_VAR_LEN & 64 \\
\hline 40790 & 939 & STATIC_VOLT_VAR_ActCrV & 1 \\
\hline 40791 & 940 & STATIC_VOLT_VAR_ModEna & 0x0001 [Enabled] \\
\hline 40792 & 941 & STATIC_VOLT_VAR_WinTms & Not implemented \\
\hline 40793 & 942 & STATIC_VOLT_VAR_RvrtTms & Not implemented \\
\hline 40794 & 943 & STATIC_VOLT_VAR_RmpTms & Not implemented \\
\hline 40795 & 944 & STATIC_VOLT_VAR_NCrV & 1 \\
\hline 40796 & 945 & STATIC_VOLT_VAR_NPt & 4 \\
\hline 40797 & 946 & STATIC_VOLT_VAR_V_SF & -2 \\
\hline 40798 & 947 & STATIC_VOLT_VAR_DeptRef_SF & -1 \\
\hline 40799 & 948 & STATIC_VOLT_VAR_RmpIncDec_SF & 0 \\
\hline 40800 & 949 & STATIC_VOLT_VAR_ActPt & 4 \\
\hline 40801 & 950 & STATIC_VOLT_VAR_DeptRef & 1 [WMax] \\
\hline 40802 & 951 & STATIC_VOLT_VAR_V1 & $41.00 \%$ VRef \\
\hline 40803 & 952 & STATIC_VOLT_VAR_VAr1 & 46.0 \\
\hline 40804 & 953 & STATIC_VOLT_VAR_V2 & $44.00 \%$ VRef \\
\hline 40805 & 954 & STATIC_VOLT_VAR_VAr2 & 0.0 \\
\hline 40806 & 955 & STATIC_VOLT_VAR_V3 & $52.00 \%$ VRef \\
\hline 40807 & 956 & STATIC_VOLT_VAR_VAr3 & 0.0 \\
\hline 40808 & 957 & STATIC_VOLT_VAR_V4 & $55.00 \%$ VRef \\
\hline 40809 & 958 & STATIC_VOLT_VAR_VAr4 & 46.0 \\
\hline
\end{tabular}

Table 6.6: Verify Volt-VAr points were transferred into SunSpec registers using OutBack Shell program. 


\subsubsection{Volt-VAr function interoperability testing analysis}

Even the user-defined points can be transferred into the SunSpec registers, but the inverter does not use those values to set a Volt-VAr curve for the inverter to operate. Therefore, the smart inverter is not fully compliant with the opModVoltVar attribute. In conclusion, the inverter does not provide a functionally correct response to this attribute.

\subsubsection{GS8048A Volt-VAr Function Testing with Grid Simulator}

Although the inverter is not compliant with IEEE 2030.5 attribute opModVoltVar, the sub-component works in the testing system. The Volt-VAr function of the inverter can be tested when the grid-simulator is connected. As defined in the GS8048A operator manual, there are three sets of voltage points for the Volt-VAr curve: the default voltage points, minimum voltage points, and maximum voltage points. The Volt-VAr testing in this section was conducted using those voltage points. The time step for voltage changes on the grid simulator was set as 2 or 5 seconds. The voltage sweep direction is from maximum to minimum and from minimum to maximum.

\subsubsection{Default Volt-VAr test}

This default Volt-VAr test explores how the inverter is sensitive to the voltage changes as the voltage does not reach the minimum and maximum bound with the default voltage set points.

\section{Default Volt-VAr test sweep voltage from low to high with $\mathbf{2}$ seconds time step}


The first test explores how the Volt-VAr function works with the minimum acceptable voltage-time changes and the sweep direction from the minimum to the maximum. The time step for voltage change is 2 seconds. The default voltage set points are summarized in the Table 6.7. The maximum reactive power is $1980 \mathrm{VAr}$, which is the maximum reactive power as the inverter export the maximum apparent power VA.

\begin{tabular}{|c|c|c|c|c|c|}
\hline Volt & V_phase & V_LL & Q(VAr) & Q_value & S=3.3kVA, PF=0.8 \\
\hline V1 & 106 & 212 & Q1 & 1980 & Q_max=sqrt(S^2-P^2) \\
\hline V2 & 114 & 228 & Q2 & 500 & Q_deadband \\
\hline V3 & 126 & 252 & Q3 & 500 & Q_deadband \\
\hline V4 & 132 & 264 & Q4 & -1980 & Q_max \\
\hline
\end{tabular}

Table 6.7: Default Volt-VAr curve points.

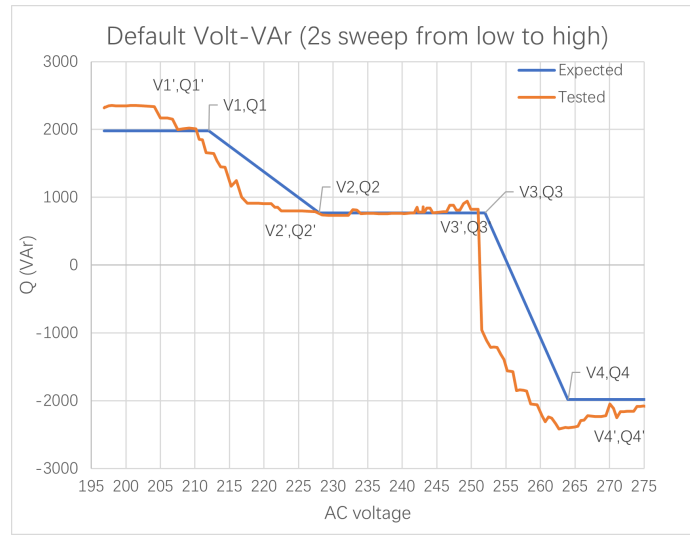

Figure 6.9: Default Volt-VAr curve sweep voltage from the minimum to maximum with 2 seconds time step.

As shown in Figure 6.9, point V1' is lower than the desired V1 point, and Q1' is a higher than the desired Q1. The dead-band is shifted to the left. The reason that the tested Volt-VAr curve has a big drop between V3 and V4 is because the reactive power within the dead-band is not zero. The direction of the reactive power has to change quickly in order to keep up the voltage changes as the voltage sweep from the V3 to V4, which the inverter is not able to do. 


\section{Default Volt-VAr test sweep voltage from high to low with $\mathbf{2}$ seconds time step}

The second test explores how the Volt-VAr function works when the voltage sweep from the maximum to the minimum with a 2 seconds time step and when the maximum reactive power is in a low condition. The GS8048A smart inverter cannot only export reactive power because the minimum power factor that can be set is 0.8 in the Volt-VAr function to provide the maximum reactive power. Therefore, active power will be exported when the inverter provides reactive power. Since the active power is continuously exported from the inverter when the Volt-VAr function is enabled, the amount of reactive power decreases to keep the fixed power factor 0.8 . The maximum reactive power of this test is 1000 VAr. That is the maximum value as the inverter export around one-third of the maximum apparent power of 3300 VA. The voltage set points of this test are summarized in the Table 6.8 .

\begin{tabular}{|c|c|c|c|c|c|}
\hline Volt & V_phase & V_LL & Q(VAr) & Q_value & Reactive power (Q) \\
\hline V1 & 106 & 212 & Q1 & 1000 & Q_max \\
\hline V2 & 114 & 228 & Q2 & 215 & Q_deadband \\
\hline V3 & 126 & 252 & Q3 & 215 & Q_deadband \\
\hline V4 & 132 & 264 & Q4 & -1000 & Q_max \\
\hline
\end{tabular}

Table 6.8: Default Volt-VAr curve points. 


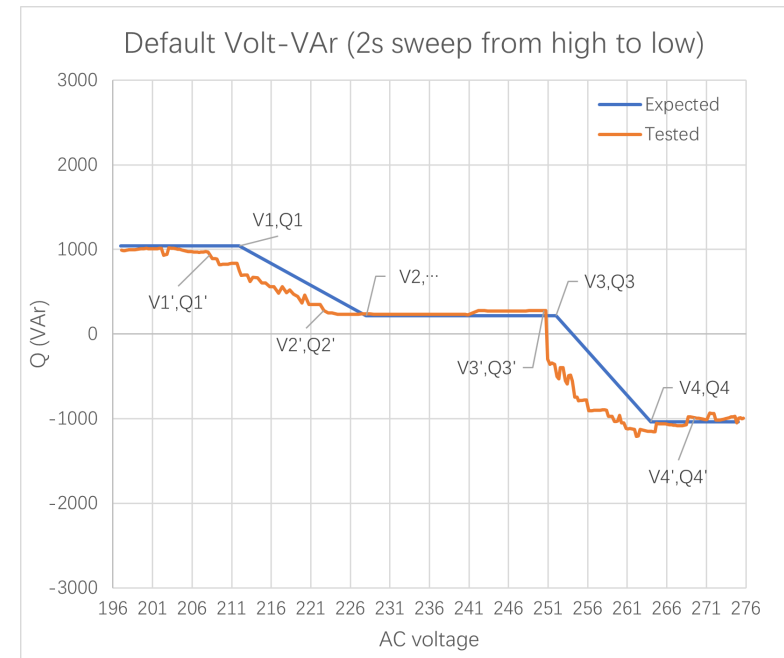

Figure 6.10: Default Volt-VAr testing sweep voltage from high to low with 2 seconds time step.

As shown in Figure 6.10, the tested Volt-VAr curve is shifted to the left compared to the expected Volt-VAr curve. The Volt-VAr control of this test behaves more accurately compared to the previous test.

\section{Default Volt-VAr test sweep voltage from low to high with 5 seconds time step}

In order to test the accuracy of the Volt-VAr function respond to a different time step of the voltage changes, the time step of this test is switched to 5 seconds. In this third test, the voltage sweeps from the minimum to the maximum. The voltage set points are summarized in the Table 6.7.

As shown in Figure 6.11, when the voltage is swept from the minimum to the maximum, the tested Volt-VAr curve mostly followes the user-defined expected curve, except for the maximum reactive power decreased due to the inverter continuously discharging, as explained in the previous test statement. 


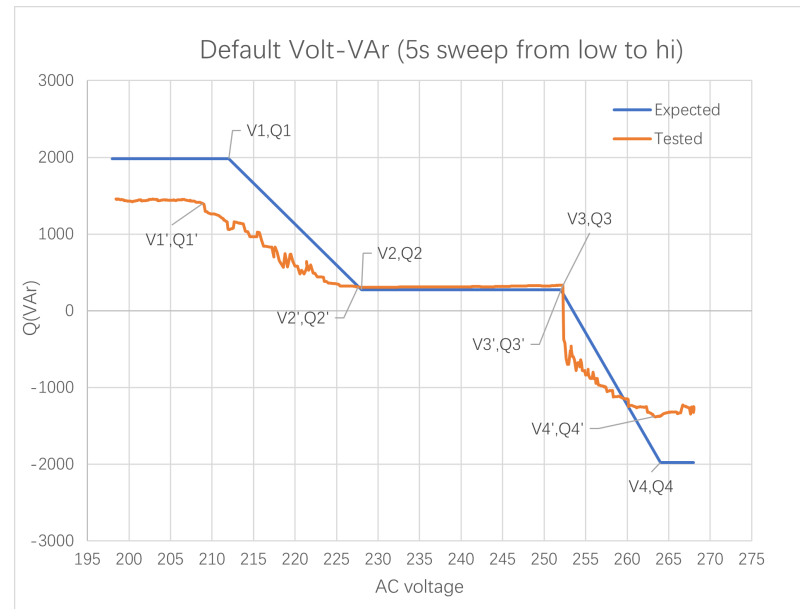

Figure 6.11: Default Volt-VAr testing sweep voltage from low to high with 5 seconds time step.

\section{Default Volt-VAr test sweep voltage from high to low with $\mathbf{5}$ seconds time step}

Similar to the third test, the fourth test of this explores how Volt-VAr function behaves when the voltage swept from the maximum to the minimum with 5 seconds time step. The voltage set points are summarized in the Table 6.7.

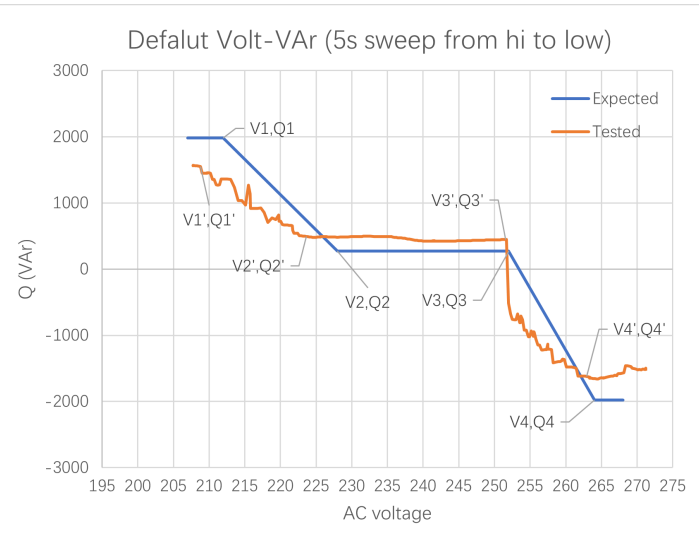

Figure 6.12: Default Volt-VAr testing sweep voltage from high to low with 5 seconds time step.

As shown in Figure 6.12, when the voltage is swept from the maximum to the minimum, the dead-band was extended. Point 1 and point 2 shifted to the left. The 
maximum reactive power was reduced due to the inverter being discharged as the test went.

\subsubsection{Minimum Volt-VAr Test}

In order to test the performance of Volt-VAr control when the voltages are set to the minimum acceptable value, the following tests were conducted. Similar to the default Volt-VAr tests, the following are also conducted with different time steps for voltages and with voltage sweep directions.

\section{Minimum Volt-VAr test sweep voltage from low to high with 2 seconds time step}

This test explores how well the Volt-VAr control performs when the voltages were set as the minimum acceptable value. The time step for voltage changes is 2 seconds and the voltage sweep direction is from the minimum to the maximum in this test. The voltage set points of this test are summarized in the Table 6.9.

\begin{tabular}{|c|c|c|c|c|}
\hline Volt & V_phase & V_LL & Q(VAr) & Q_value \\
\hline V1 & 98 & 196 & Q1 & 1980 \\
\hline V2 & 106 & 212 & Q2 & 500 \\
\hline V3 & 114 & 228 & Q3 & 500 \\
\hline V4 & 116 & 232 & Q4 & -1980 \\
\hline
\end{tabular}

Table 6.9: Minimum Volt-VAr curve points.

As shown in Figure 6.13, the dead-band of the test Volt-VAr curve is shifted to the left compared to the expected curve. The maximum inductive and capacitive power were provided when the sweep voltage hits the V1 and V4 points on tested curve.

\section{Minimum Volt-VAr test sweep voltage from high to low with 2 seconds time step}




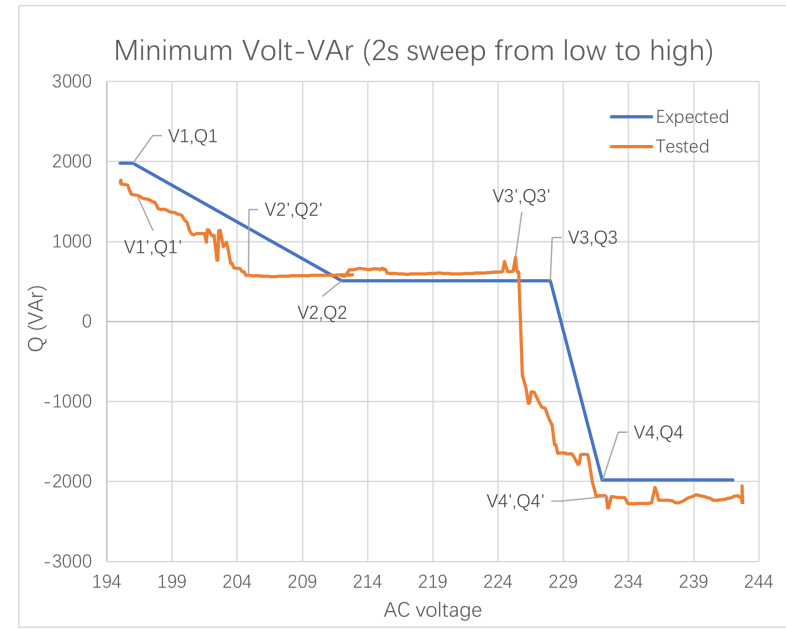

Figure 6.13: Minimum Volt-VAr testing sweep voltage from low to high with 2 seconds time step.

Different from the previous test, the voltage sweep direction of this test was changed from maximum to minimum. The voltage step time is set as 2 seconds. Moreover, this test also explores the performance of the Volt-VAr control when the maximum reactive power is provided. The voltage set points are summarized in the Table 6.10.

\begin{tabular}{|c|c|c|c|c|}
\hline Volt & V_phase & V_LL & Q(VAr) & Q_value \\
\hline V1 & 98 & 196 & Q1 & 1000 \\
\hline V2 & 106 & 212 & Q2 & 215 \\
\hline V3 & 114 & 228 & Q3 & 215 \\
\hline V4 & 116 & 232 & Q4 & -1000 \\
\hline
\end{tabular}

Table 6.10: Minimum Volt-VAr curve points.

As shown in Figure 6.14, the dead-band of the tested Volt-VAr curve is shifted to the left. The maximum inductive and capacitive power provided by the inverter has a 500 VAr difference when the voltage swept at (V1',Q1') and (V4',Q4') . 


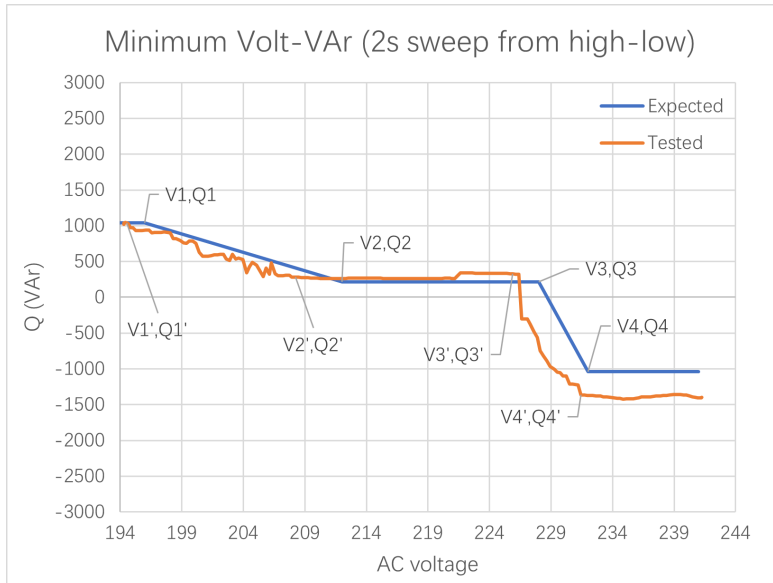

Figure 6.14: Minimum Volt-VAr testing sweep voltage from high to low with 2 seconds time step.

\section{Minimum Volt-VAr test sweep voltage from low to high with 5 seconds time step}

This test explores the performance of the Volt-VAr control as the voltage-time step extends to 5 seconds, and the voltage points are set close to the minimum acceptable value. The voltage sweep direction of this test is from the minimum to the maximum. The voltage set points are summarized in Table 6.10.

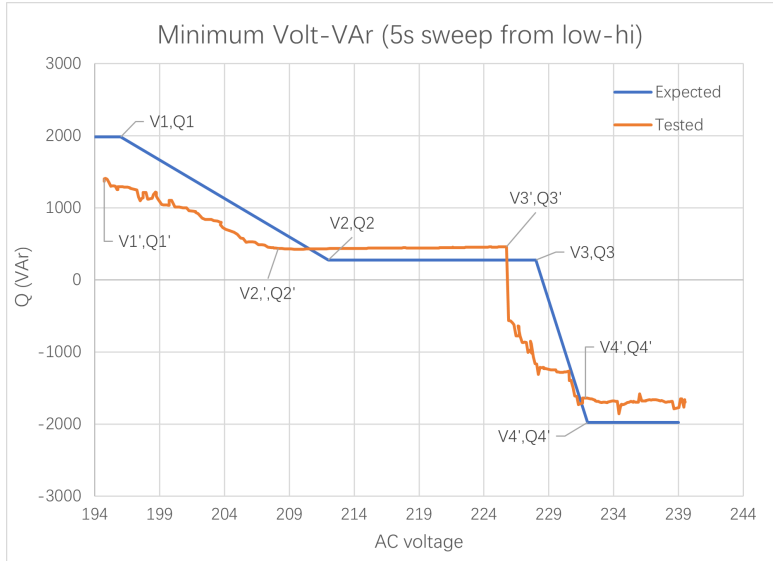

Figure 6.15: Minimum Volt-VAr testing sweep voltage from low to high with 5 seconds time step.

As shown in Figure 6.15, the performance of the Volt-VAr curve does not improve 
compared to the Figure 6.13. The dead-band of the tested Volt-VAr curve is shifted to the left. The maximum reactive power on the tested curve is lower than the expected curve due to the inverter was under discharging as the test went.

\section{Minimum Volt-VAr test sweep voltage from high to low with 5 seconds time step}

This test explores the performance of the Volt-VAr control when the voltage-sweep direction is from maximum to the minimum. The voltage time step was set as 5 seconds. The voltage set points are summarized in Table 6.10 .

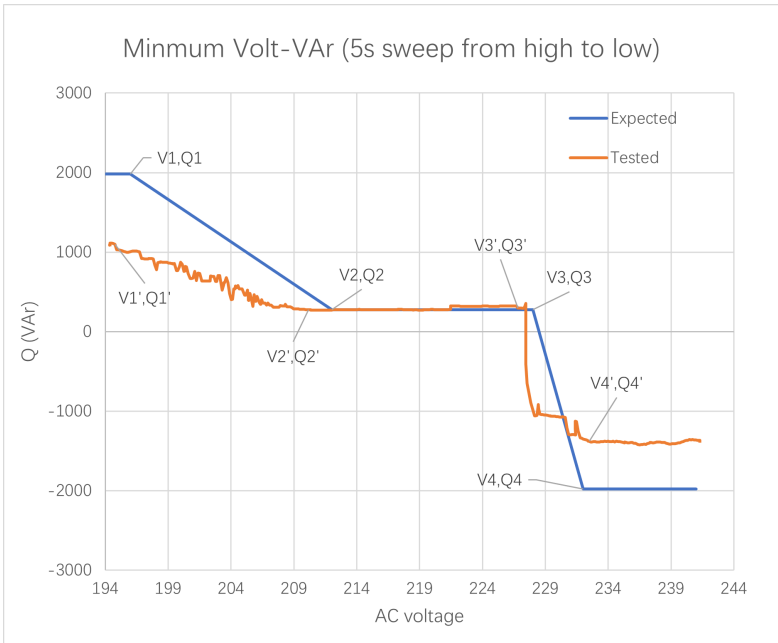

Figure 6.16: Minimum Volt-VAr testing sweep voltage from high to low with 5 seconds time step.

As shown in Figure 6.16, when the voltage sweep is from maximum to minimum, point (V2', Q2') shifts to the left. Other voltage points on the tested curve match with user-defined points. 


\subsubsection{Maximum Volt-VAr curve Test}

In order to test the performance of Volt-VAr control when the voltages are set to the maximum acceptable value, the following tests were conducted. Similar to the default Volt-VAr tests, the followings are also conducted with different voltage sweep times and directions.

\section{Maximum Volt-VAr test sweep voltage from low to high with 2 seconds time step}

This test explores how well the Volt-VAr control was conducted when the voltage points were set as the maximum acceptable value with voltage-time step 2 seconds and sweep direction from minimum to maximum. The voltage set points of this test are summarized in the Table 6.11.

\begin{tabular}{|c|c|c|c|c|}
\hline Volt & V_phase & V_LL & Q(VAr) & Q_value \\
\hline V1 & 124 & 248 & Q1 & 1980 \\
\hline V2 & 126 & 252 & Q2 & 500 \\
\hline V3 & 132 & 264 & Q3 & 500 \\
\hline V4 & 139 & 278 & Q4 & -1980 \\
\hline
\end{tabular}

Table 6.11: Maximum Volt-VAr curve points.

As shown in the Figure 6.17, the tested maximum Volt-VAr function has a noticeable difference compared to the expected test result. The Volt-VAr control did not follow the user defined voltage points.

\section{Maximum Volt-VAr test sweep voltage from high to low with $\mathbf{2}$ seconds time step}

Different from the previous test, the voltage-sweep direction of this test was changed from the maximum to the minimum. The voltage step time is set as 2 seconds. More- 


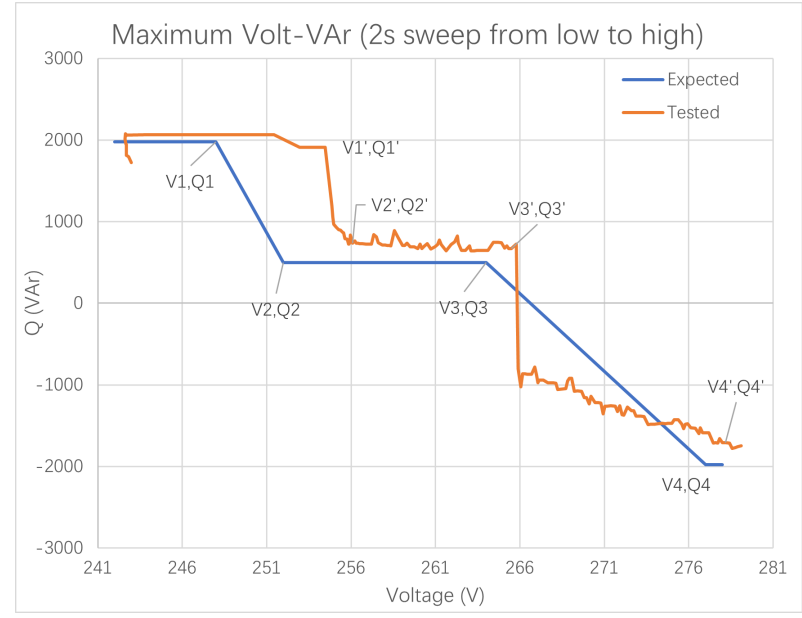

Figure 6.17: Maximum Volt-VAr testing sweep voltage from low to high with 2 seconds time step.

over, this test also explores the performance of the Volt-VAr control with maximum acceptable voltage when the maximum reactive power is reduced. The voltage set points are summarized in the Table 6.12.

\begin{tabular}{|c|c|c|c|c|}
\hline Volt & V_phase & V_LL & Q(VAr) & Q_value \\
\hline V1 & 124 & 248 & Q1 & 1000 \\
\hline V2 & 126 & 252 & Q2 & 215 \\
\hline V3 & 132 & 264 & Q3 & 215 \\
\hline V4 & 139 & 278 & Q4 & -1000 \\
\hline
\end{tabular}

Table 6.12: Maximum Volt-VAr curve points.

As shown in Figure 6.18, point (V1', Q1') and (V2', Q2') were shifted to the left as the voltage swept from the maximum to the minimum. Point (V3', Q3') and (V4', Q4') on the tested curve followed the user-defined voltage points and the maximum reactive power was provided as the swept voltage hit point 1 and point 4 . The dead-band of the tested curve in this test is extended due to point 2 being shifted.

\section{Maximum Volt-VAr test sweep voltage from low to high with 5 seconds time step}




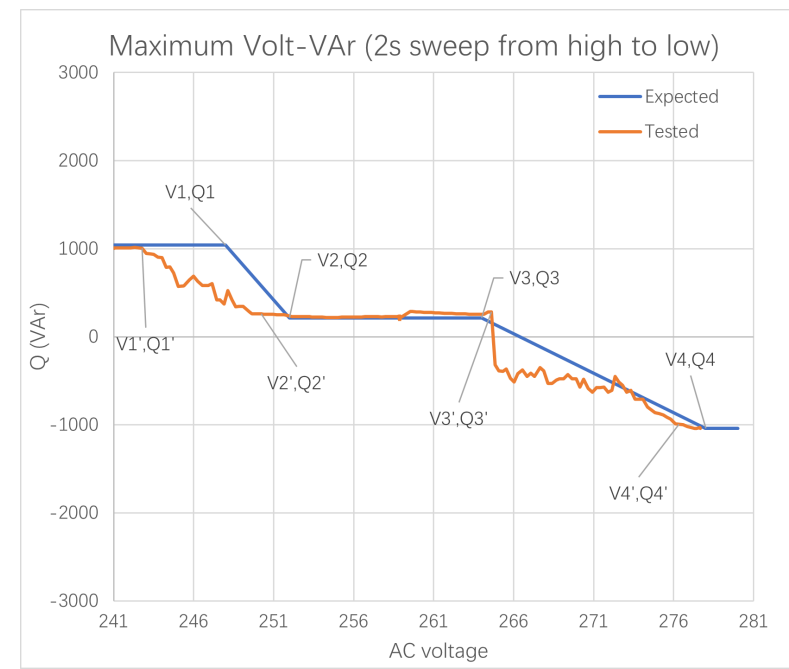

Figure 6.18: Maximum Volt-VAr testing sweep voltage from high to low with 2 seconds time step.

This test explores the performance of the Volt-VAr control as the voltage-time step extended to 5 seconds, and the voltage points were set close to the maximum acceptable value. The voltage sweep direction of this test is from minimum to maximum. The voltage set points are summarized in Table 6.12.

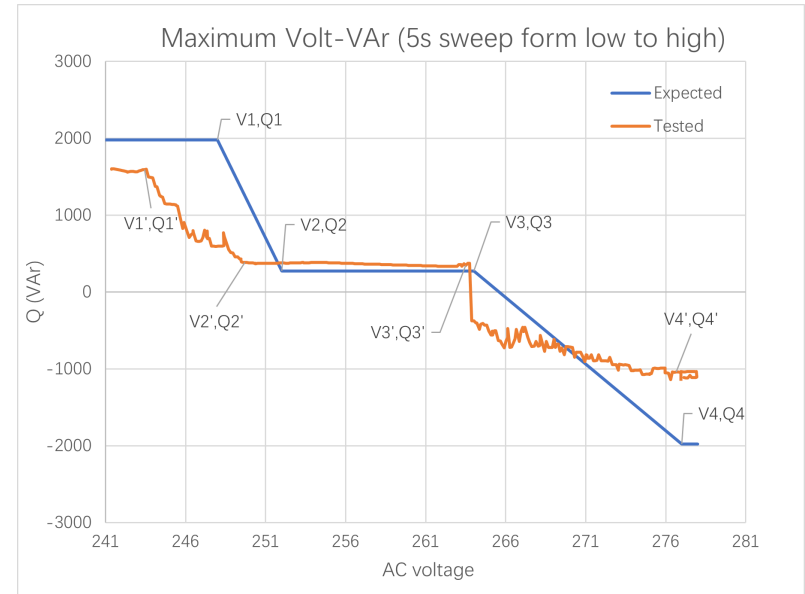

Figure 6.19: Maximum Volt-VAr testing sweep voltage from low to high with 5 seconds time step.

As shown in Figure 6.19, point (V1', Q1') and (V2', Q2') were shifted to the left as 
the voltage swept from the minimum to the maximum. Point (V3", Q3') and (V4, Q4') on the tested curve followed the user-defined voltage points. The dead-band of the tested curve in this test is extended due to point 2 being shifted to the left. There is a 500 VAr different between the capacitive VAr and inductive VAr.

\section{Maximum Volt-VAr test sweep voltage from high to low with 5 seconds time step}

This test explores the performance of the Volt-VAr control when the voltage-sweep direction was changed: from the maximum to the minimum. The voltage time step was set as 5 seconds. The voltage set points are summarized in Table 6.12 .

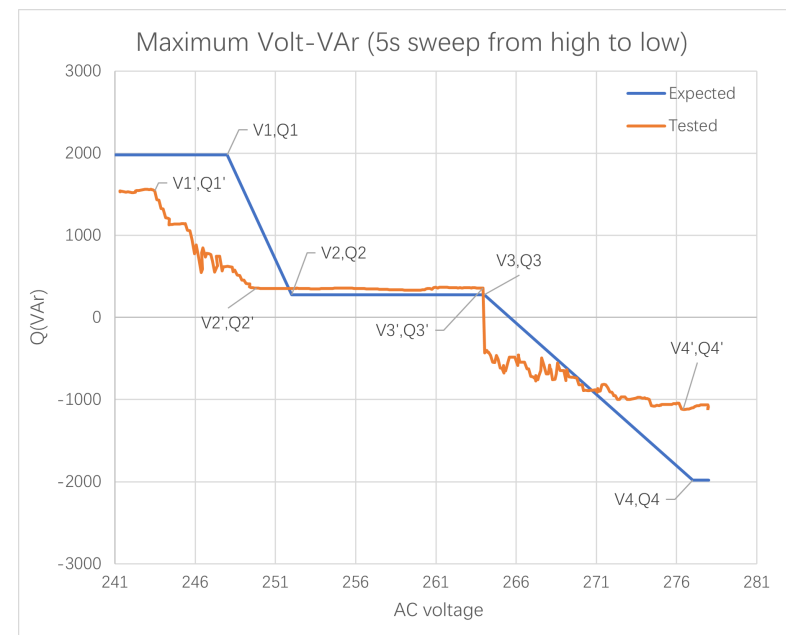

Figure 6.20: Maximum Volt-VAr testing sweep voltage from high to low with 5 seconds time step.

As shown in Figure 6.20, point (V1', $\left.1^{\prime}\right)$ and (V2', Q2') were shifted to the left as the voltage swept from the maximum to the minimum. Point (V3', Q3') and (V4', Q4') on the tested curve followed the user-defined voltage points. The dead-band of the tested curve in this test is extended due to point 2 being shifted to the left. There 
is a 500 VAr different between the capacitive VAr and inductive VAr. Based on the observation, there is no improvement compared to the previous test.

\subsubsection{Volt-VAr function Sub-component Testing Analysis}

Based on the above testing result, the inverter testing system has the ability to test both the lower band and higher band of the voltages in the Volt-VAr function. The inverter has the ability to test the Volt-VAr function with different voltage sweep directions and is able to test the Volt-VAr function with different voltage time step changes even the GS 8048A smart inverter not always follow the user-defined curve very well. 


\subsection{Frequency Watt Function Testing}

Similar to the voltage, frequency is also an essential factor of a reliable electric grid. However, unlike the Volt-VAr function control where the inverter can provide autonomous bi-directional reactive power, most commercial and residential inverters sold today can only provide frequency-Watt control for over-frequency situations. Under-frequency control in the solar PV inverter is possible, but it will require the inverter to operate below the minimum available power from the PV solar panels. This new control is currently underdevelopment and has not been widely adopted in residential and commercial inverters [30]. Therefore, the desired frequency-Watt control curve for over-frequency events will look as shown in Figure 6.21. As the frequency increases, the inverter will reduce exporting power to the grid. But it will always export its maximum set power as the frequency is below the nominal value. According to the SIWG Phase 3 DER Functions [30], "The curve is assumed to extend horizontally to the left below the lowest point and to the right above the highest point in the array."

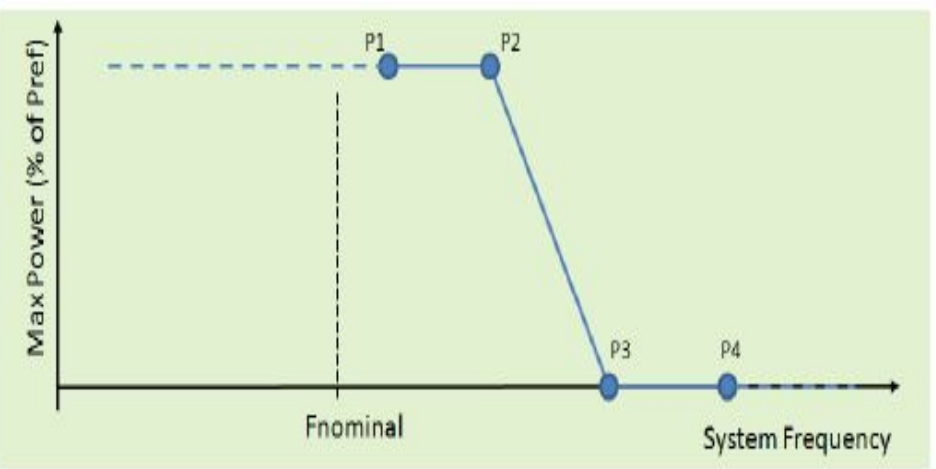

Figure 6.21: Frequency-Watt Curve for over-frequency. [30] 
As defined in IEEE 2030.5, the opModFreqWatt attribute requires the frequency-Watt curve-based function be composed of pairs of points in an array. The "x" value in each pair of points defines the frequency in $\mathrm{Hz}$ and the "y" value defines a real power level in the percentage of maximum power (WMax). For an inverter capable of reacting to under-frequency situations, the frequency-Watt curve will be as shown in Figure 6.22. Point 1 specifies the maximum power that the inverter needs to export when the grid frequency is blow the nominal value. Points 2 and 3 specify the dead-band of the frequency-Watt curve. As shown in the Figure 6.22. the ideal export power from the inverter in dead-band is 0 , but in a real case, it is the minimum export power that the inverter provides. This has been verified by the test results shown in the later section. Point 4 specifies the maximum power that the inverter can absorb when the grid frequency is above the nominal value.

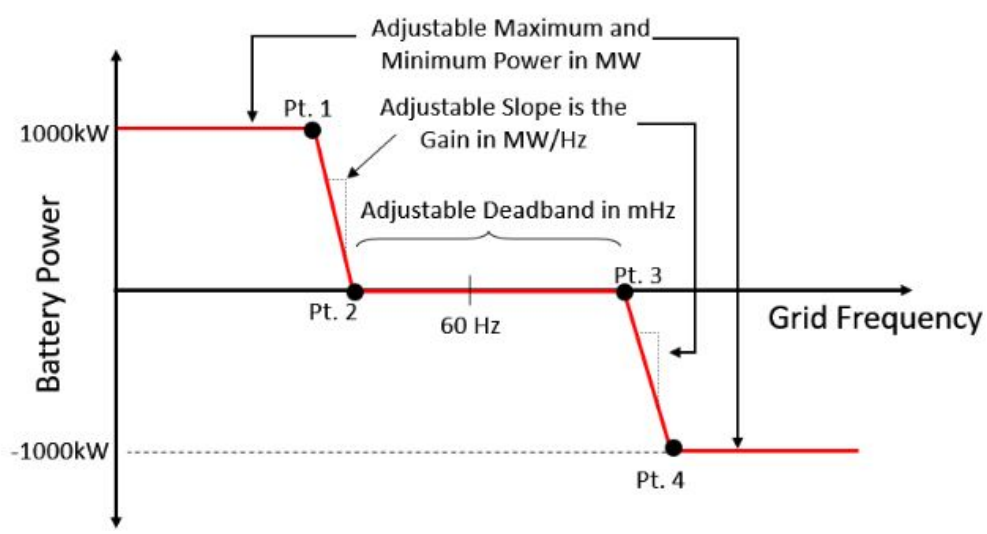

Figure 6.22: The frequency-Watt curve used to adjust the frequency response characteristics of the BIS [31]. 


\subsubsection{Verification of Writing Frequency-Watt Curve Points}

In the DER program, point $1(\mathrm{~Hz} 1, \mathrm{~W} 1)$ was set as $(57,80),(\mathrm{Hz} 2, \mathrm{~W} 2)$ and $(\mathrm{Hz} 3, \mathrm{~W} 3)$ were set as $(58,0)$ and $(60,0)$, and $(\mathrm{Hz} 4, \mathrm{~W} 4)$ was set as $(62,-80)$. Since the $\mathrm{W} 1$ and $\mathrm{W} 4$ are percentage values, $W 1=80 \% \times 720 W=576 W$ and $W 4=-80 \% \times 720=-576 W$.

Therefore, the expected bi-directional frequency-Watt curve will be like Figure 6.23.

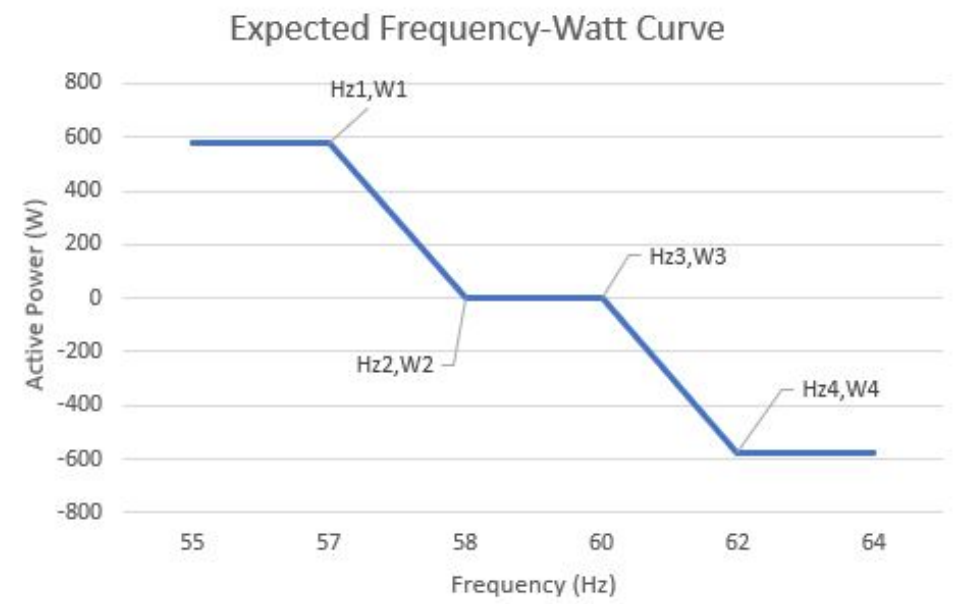

Figure 6.23: User-defined expected bi-directional frequency-Watt curve.

Because the bi-directional frequency-Watt curve control has not been implemented in the GS8048A smart inverter, the SunSpec registesr "W1", "W2", "W3", "W4" in the block 134 "Freq-Watt Curve" cannot be written into the AXS port. Also, the SunSpec registers "Hz 1" and "Hz 2" only allow small numbers to be written into them, so the maximum frequency numbers that can be written into the registers are $5 \mathrm{~Hz}$. Other frequency points:"Hz 3 " and "Hz 4" do not allow values to be transferred into them. The value defined in the IEEE 2030.5 attribute is firstly scaled up to 100 times in the DER program and then scale down to 
the normal value, so the value stored in the SunSpec register should be $57 \mathrm{~Hz}, 59 \mathrm{~Hz}, 61 \mathrm{~Hz}$ and $63 \mathrm{~Hz}$. Table 6.13 summarized the test result of writing frequency values into SunSpec registers using DER program.

\begin{tabular}{|c|c|c|c|c|}
\hline IEEE 2030.5 attribute & Transfer Value & $\begin{array}{c}\text { SunSpec } \\
\text { Register }\end{array}$ & $\begin{array}{c}\text { SunSpec Register } \\
\text { Status }\end{array}$ & $\begin{array}{c}\text { Success or } \\
\text { Failure }\end{array}$ \\
\hline $\begin{array}{c}\text { opmodFreqWatt } \\
\text { CurveData fw[0].x=57 }\end{array}$ & WritePoint: 41057,5700 & Hz1 & Illegal Data Value & Failure \\
\hline $\begin{array}{c}\text { opmodFreqWatt } \\
\text { CurveData fw[0].x=5 }\end{array}$ & WritePoint: 41057,500 & Hz1 & No error message & Success \\
\hline $\begin{array}{c}\text { opmodFreqWatt } \\
\text { CurveData fw[0].x=59 }\end{array}$ & WritePoint: 41059,5900 & Hz2 & Illegal Data Value & Failure \\
\hline $\begin{array}{c}\text { opmodFreqWatt } \\
\text { CurveData fw[0].x=61 }\end{array}$ & WritePoint: 41061,6100 & Hz3 & Illegal Data Value & Failure \\
\hline $\begin{array}{c}\text { opmodFreqWatt } \\
\text { CurveData fw[0].x=5 }\end{array}$ & WritePoint: 41061,500 & Hz3 & No error message & Success \\
\hline $\begin{array}{c}\text { opmodFreqWatt } \\
\text { CurveData fw[0].x=63 }\end{array}$ & WritePoint: 41063,6300 & Hz4 & Illegal Data Value & Failure \\
\hline
\end{tabular}

Table 6.13: Using DER program to write values into SunSpec registers $\mathrm{Hz} 1$ to $\mathrm{Hz} 4$.

User-defined active power values cannot be written into the SunSpec registers from "W1" to "W4". Table 6.14 summarized the test result of writing values into SunSpec register from "W1" to "W4" after running DER program. The values stored in the SunSpec register $\mathrm{W} 1$ and W3 are always 100\%. The possible explanation of this problem is the SunSpec block 134 (frequency-Watt Curve) has not been fully implemented in the AXS port.

\begin{tabular}{|c|c|c|c|c|}
\hline IEEE 2030.5 attribute & Transfer Value & $\begin{array}{c}\text { SunSpec } \\
\text { Register name }\end{array}$ & $\begin{array}{c}\text { SunSpec Resiter } \\
\text { Stored Value }\end{array}$ & $\begin{array}{c}\text { SunSpec } \\
\text { Register Status }\end{array}$ \\
\hline $\begin{array}{c}\text { opmodFreqWatt } \\
\text { CurveData fw[0].y=80 }\end{array}$ & WritePoint: 41058, 80 & W1 & 100 & 100 \\
\hline $\begin{array}{c}\text { opmodFreqWatt } \\
\text { CurveData fw[1].y=0 }\end{array}$ & WritePoint: 41060, 0 & W2 & 0 & 0 \\
\hline $\begin{array}{c}\text { opmodFreqWatt } \\
\text { CurveData fw[2].y=0 }\end{array}$ & WritePoint: 41062, 0 & W3 & -100 & -100 \\
\hline $\begin{array}{c}\text { opmodFreqWatt } \\
\text { CurveData fw[3].y=-80 }\end{array}$ & WritePoint: 41064, -80 & W4 & 0 & 0 \\
\hline
\end{tabular}

Table 6.14: Using DER program to write values into SunSpec registers W1 to W4.

To make sure the test result of the DER program are correct, the OutBack Shellprogram was used to double check the work. Field number 1178 represents register "Hz 1", by that 
analogy, field number 1184 is register "Hz 4". Similarly, field number 1179 represents register "W 1" and field number 1185 represents register "W 4". Table 6.15 summarize the test result of writing SunSpec values for frequency-Watt curve using the Outback Shell program.

\begin{tabular}{|c|c|c|c|c|c|}
\hline $\begin{array}{c}\text { Register } \\
\text { Address } \\
\text { in } \\
\text { AXS port }\end{array}$ & $\begin{array}{c}\text { SunSpec } \\
\text { Register offset } \\
\text { in } \\
\text { AXS port }\end{array}$ & Register Name & Value & $\begin{array}{c}\text { Transfer } \\
\text { Value }\end{array}$ & Error Message \\
\hline 41044 & 1165 & FREQ_WATT_DID & 134 & N/A & N/A \\
\hline 41045 & 1166 & FREQ_WATT_LEN & 68 & N/A & N/A \\
\hline 41046 & 1167 & FREQ_WATT_ActCrv & 0 & N/A & N/A \\
\hline 41047 & 1168 & FREQ_WATT_ModEna & 0 D0000 & N/A & N/A \\
\hline 41048 & 1169 & FREQ_WATT_WinTms & Not implemented & N/A & N/A \\
\hline 41049 & 1170 & FREQ_WATT_RvrtTms & 0 seconds & N/A & N/A \\
\hline 41050 & 1171 & FREQ_WATT_RmpTms & Not implemented & N/A & N/A \\
\hline 41051 & 1172 & FREQ_WATT_NCrv & 1 & N/A & N/A \\
\hline 41052 & 1173 & FREQ_WATT_NPt & 4 & N/A & N/A \\
\hline 41053 & 1174 & FREQ_WATT_Hz_SF & -2 & N/A & N/A \\
\hline 41054 & 1175 & FREQ_WATT_W_SF & 1 & N/A & N/A \\
\hline 41055 & 1176 & FREQ_WATT_RmpIncDec_SF & 0 & N/A & N/A \\
\hline 41056 & 1177 & FREQ_WATT_ActPt & 4 & N/A & N/A \\
\hline 41057 & 1178 & FREQ_WATT_Hz1 & $5.00 \mathrm{~Hz}$ & 50 & Write Success \\
\hline 41058 & 1179 & FREQ WATT_Hz1 & $8.00 \mathrm{~Hz}$ & 80 & Illegal Data Value \\
\hline 41058 & 1179 & FREQ WATT_W1 & $100 \% \mathrm{WRef}$ & 80 & Illegal Data Value \\
\hline 41059 & 1180 & FREQ WATT_Hz2 & $20.00 \mathrm{~Hz}$ & 50 & Illegal Data Value \\
\hline 41060 & 1181 & FREQ_WATT_W2 & $0 \% \mathrm{WRef}$ & 0 & Illegal Data Value \\
\hline 41061 & 1182 & FREQ_WATT_Hz3 & $5 \mathrm{~Hz}$ & 50 & Write Success \\
\hline 41062 & 1183 & FREQ_WATT_W3 & $-100 \% \mathrm{WRef}$ & 0 & Illegal Data Value \\
\hline 41063 & 1184 & FREQ_WATT_Hz4 & $20.00 \mathrm{~Hz}$ & 50 & Illegal Data Value \\
\hline 41064 & 1185 & FREQ_WATT_W4 & $0 \% \mathrm{WRef}$ & -80 & fllegal Data Value \\
\hline
\end{tabular}

Table 6.15: Verify frequency-Watt points were transferred into SunSpec registers using OutBack Shell program.

\subsubsection{Frequency-Watt function interoperability testing analysis}

Based on the test results, the testing system discovers that only SunSpec register $\mathrm{Hz} 1$ and $\mathrm{Hz} 3$ allow the value transferred into. However, the allowable frequency value is too small, which cannot be used for setting a freq-Watt Curve for an inverter to operate. Therefore, the smart inverter GS8048A is not compliant with IEEE 2030.5 opModFreqWattattributes. In 
conclusion, the smart inverter does not provide a functionally correct response to the IEEE 2030.5 opModFreqWattattributes.

\subsubsection{Frequency-Watt Sub-component testing preparation}

Although the frequency-Watt control of the inverter cannot be verified by the user set points written in opModFreqWatt attributes, we still want to show that our test system sub-components works, specifically when the inverter is connected to the grid simulator. According to the GS8048A operator manual, the inverter supports the bi-directional frequency-Watt control, but the user cannot set points for the frequency-Watt curve like the Volt-VAr function as shown Table 6.16.

\begin{tabular}{|c|c|c|c|c|c|}
\hline \multicolumn{3}{|l|}{ Item } & Default & Minimum & Maximum \\
\hline \multirow{7}{*}{ Frequency Watt } & \multirow{2}{*}{ Start Freq } & High & $60.05 \mathrm{~Hz}$ & $60.05 \mathrm{~Hz}$ & $65.00 \mathrm{~Hz}$ \\
\hline & & Low & $59.75 \mathrm{~Hz}$ & $55.00 \mathrm{~Hz}$ & $59.95 \mathrm{~Hz}$ \\
\hline & \multirow{2}{*}{ Gradient } & High & $250 \% / \mathrm{Hz}$ & $5 \% / \mathrm{Hz}$ & $250 \% / \mathrm{Hz}$ \\
\hline & & Low & $133 \% / \mathrm{Hz}$ & $5 \% / \mathrm{Hz}$ & $250 \% / \mathrm{Hz}$ \\
\hline & \multirow{2}{*}{$\begin{array}{l}\text { Re-Start } \\
\text { Freq }\end{array}$} & High & $60.05 \mathrm{~Hz}$ & $60.00 \mathrm{~Hz}$ & $64.90 \mathrm{~Hz}$ \\
\hline & & Low & $59.85 \mathrm{~Hz}$ & $55.10 \mathrm{~Hz}$ & $60.00 \mathrm{~Hz}$ \\
\hline & \multicolumn{2}{|c|}{ Re-Start Period } & $1 \mathrm{~min}$ & $0 \mathrm{~min}$ & $30 \mathrm{mins}$ \\
\hline
\end{tabular}

Table 6.16: Control parameters of the Freq-Watt function in GS 8048A smart inverter

As described in the Grid Support function in the operator manual, users can only set the two start points for the frequency-Watt function, which are the start frequency high point and start frequency low point. The gradient is the change rate of active power as frequency changes per Hz. A smaller value of the gradient will lead to a lower value of start frequency low and a higher value of start frequency high. The gradient value set for the frequency-Watt function is $5 \% / \mathrm{Hz}$. To enable the frequency-Watt function, the start freq high and low values should be in the range of re-start freq high and low values. The re-start 
period specifies the time that the frequency-Watt function will be re-enabled once the start freq high and low values are set beyond the range of the re-start freq high and low values.

The frequency-Watt function testing in this section attempts test the function behavior as the frequency hits the higher band and lower band. Hence, the lowest frequency that can be set as tested with the MATE3s is $55 \mathrm{~Hz}$ and the highest frequency that can be set is $65 \mathrm{~Hz}$.

\subsubsection{GS8048A Frequency-Watt Function Testing with Grid Simulator}

Since the GS8048A does not support bi-directional autonomously frequency-Watt control responding to both over-frequency and under-frequency, the following tests are focused on the frequency-Watt control responding to the over-frequency situations. In order to test the sensitivity of the frequency-Watt function to the frequency changes, the following tests used 2 seconds and 5 seconds for the frequency step changes. Moreover, the different frequency swept direction also applied to the frequency-Watt function.

\subsubsection{Frequency-Watt testing with $61 \mathrm{~Hz}$ and $58 \mathrm{~Hz}$}

In this section, the frequency-Watt tests used $61 \mathrm{~Hz}$ as the high start frequency, which defines the point where the inverter needs to reduce exporting power reacting to the higher frequency. $58 \mathrm{~Hz}$ was chosen as the low start frequency, which defines the lowest frequency point where the inverter has to export active power. Other frequency points in the range of $55 \mathrm{~Hz}$ and $65 \mathrm{~Hz}$ can also been selected. $55 \mathrm{~Hz}$ is the minimum frequency that inverter can operate with frequency-Watt function and $65 \mathrm{~Hz}$ is the maximum frequency that inverter can operate with the frequency-Watt function. 


\section{Frequency-Watt test sweep from low frequency to high frequency $(58 \mathrm{~Hz}-61 \mathrm{~Hz})$}

with 2 seconds time step

This test explores the performance of the frequency-Watt control with 2 seconds time step. The frequency sweep direction is from minimum to maximum. In this test, the maximum export power was set as half of the maximum capacity of the inverter. The expected curve points for $58 \mathrm{~Hz}$ (f1) and $61 \mathrm{~Hz}$ (f2) are summarized in Table 6.17. The point 3 (f3, W3) is the frequency that the inverter needs to export minimum active power to the grid as frequency increases. This point applies to the rest of frequency-Watt testing as well.

\begin{tabular}{|c|c|c|c|}
\hline Frequency & Hz & P & Expected Watt \\
\hline f1 & 58 & W1 & 1650 \\
\hline f2 & 61 & W2 & 1650 \\
\hline f3 & 64 & W3 & 441 \\
\hline
\end{tabular}

Table 6.17: Frequency-Watt curve points with $58 \mathrm{~Hz}$ and $61 \mathrm{~Hz}$ sweep from the minimum to the maximum frequency.

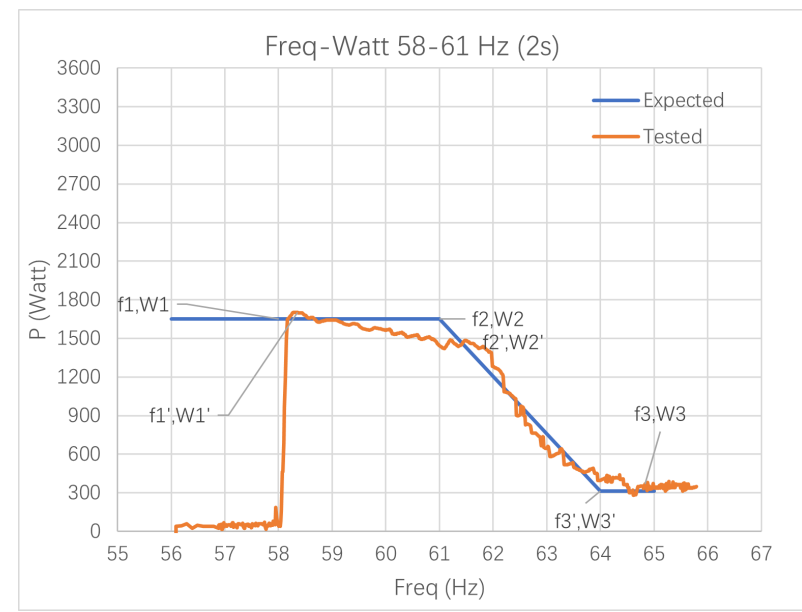

Figure 6.24: Frequency-Watt testing $(58 \mathrm{~Hz}-61 \mathrm{~Hz})$ sweep from low frequency to high frequency with 2 seconds time step and half power capacity. 
As shown in Figure 6.24, the low start frequency point $58 \mathrm{~Hz}$ and high start frequency point $61 \mathrm{~Hz}$ were shifted to the left. The inverter stops exporting the maximum power at the low start frequency. The inverter reaches the minimum point to export active power at around $65 \mathrm{~Hz}$.

\section{Frequency-Watt test sweep from high frequency to low frequency $(61 \mathrm{~Hz}-58 \mathrm{~Hz})$} with 2 seconds time step

This test explores the performance of the frequency-Watt control with different frequency swept directions compared to the previous test. The frequency step change was set as 2 seconds. The frequency was swept from the maximum value to the minimum value. Moreover, this test also explores the effect brings to the frequency-Watt function when the maximum export power is set as the full capacity of the inverter. The expected curve points for $58 \mathrm{~Hz}$ (f1) and $61 \mathrm{~Hz}$ (f2) are summarized in the Table 6.18 .

\begin{tabular}{|c|c|c|c|}
\hline Frequency & Hz & P & Expected Watt \\
\hline f1 & 58 & W2 & 3600 \\
\hline f2 & 61 & W3 & 3600 \\
\hline f3 & 64 & W4 & 300 \\
\hline
\end{tabular}

Table 6.18: Frequency-Watt curve points with $58 \mathrm{~Hz}$ and $61 \mathrm{~Hz}$ sweep from the maximum to the minimum frequency.

As shown in Figure 6.18, the low start frequency point $58 \mathrm{~Hz}$ and high start frequency point $61 \mathrm{~Hz}$ were shifted to the right. The inverter stops exporting the maximum power at the low start frequency. The inverter reaches the minimum point to export active power at $65 \mathrm{~Hz}$. 


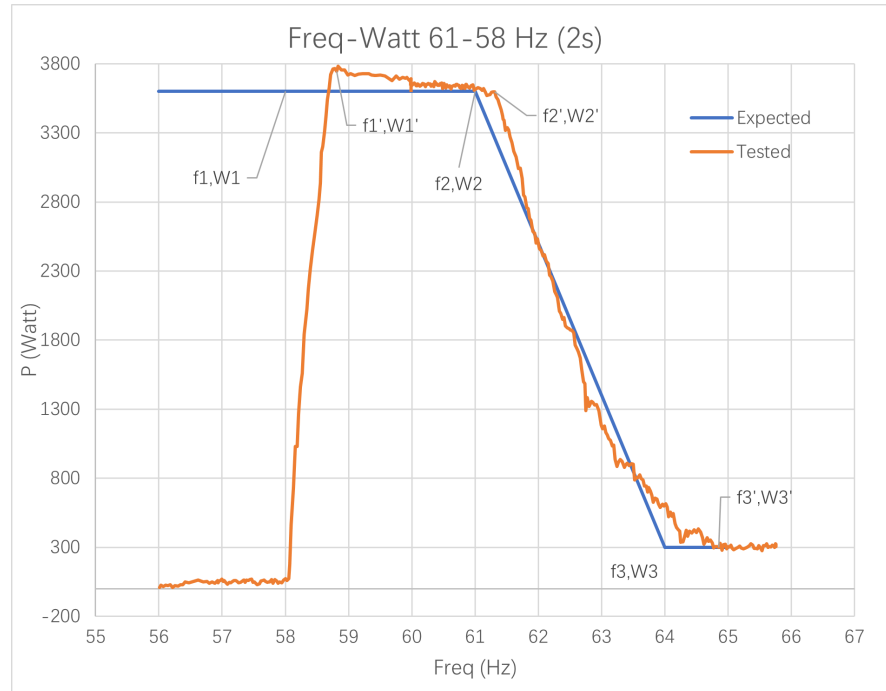

Figure 6.25: Frequency-Watt testing $(61 \mathrm{~Hz}-58 \mathrm{~Hz})$ sweep from high frequency to high frequency with 2 seconds time step and maximum power capacity.

\section{Frequency-Watt test sweep from low frequency to high frequency $(58 \mathrm{~Hz}-61 \mathrm{~Hz})$} with 5 seconds time step

In order to test the accuracy of the frequency-Watt function respond to the different time steps of the frequency changes, the time step of this test is switched to 5 seconds. Within the test, the frequency sweeps from the minimum to the maximum. The voltage set points are summarized in the Table 6.18. The maximum export power was set as full capacity of the inverter.

As shown in Figure 6.26, the low start frequency and high start frequency points on the tested curve match with the expected low start frequency, $58 \mathrm{~Hz}$, and high start frequency, $61 \mathrm{~Hz}$. The inverter reaches the minimum point to export active power at $65 \mathrm{~Hz}$. 


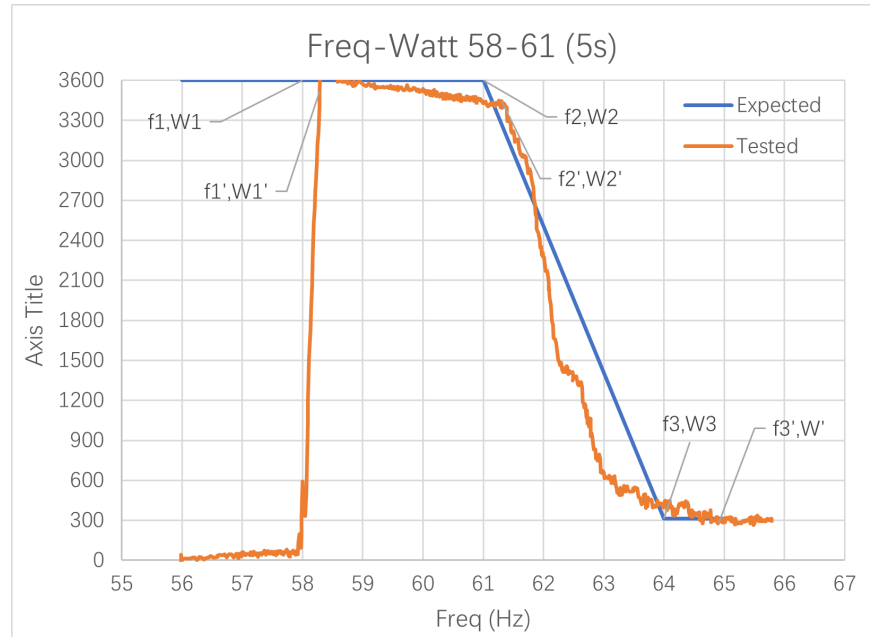

Figure 6.26: Frequency-Watt testing $(58 \mathrm{~Hz}-61 \mathrm{~Hz})$ sweep from low frequency to high frequency with 5 seconds time step and maximum power capacity.

\section{Frequency-Watt test sweep from high frequency to low frequency $(61 \mathrm{~Hz}-58 \mathrm{~Hz})$}

\section{with 5 seconds time step}

Similar to the previous test, this test explores how the frequency-Watt function behaves

when the frequency sweeps from maximum to minimum with 5 seconds time step.

The voltage set points are summarized in the Table. 6.18.

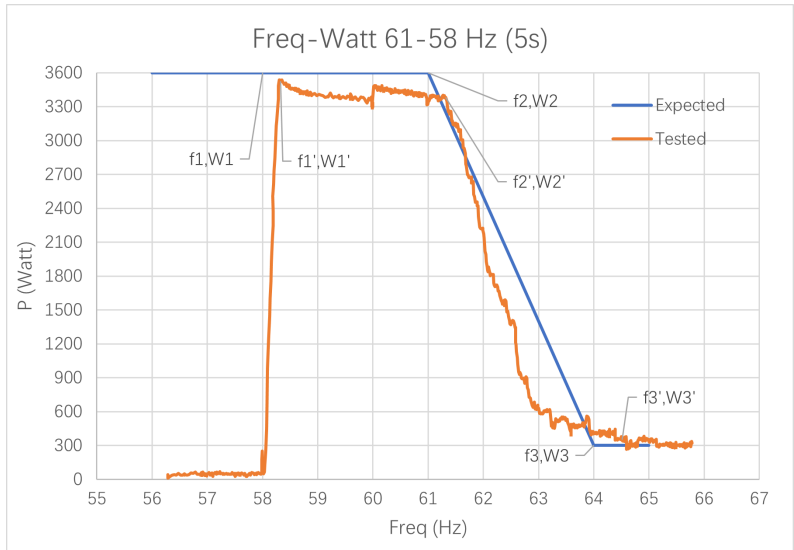

Figure 6.27: Frequency-Watt testing $(58 \mathrm{~Hz}-61 \mathrm{~Hz})$ sweep from low frequency to high frequency with 5 seconds time step and maximum power capacity. 
As shown in Figure 6.27, the low start frequency point, $58 \mathrm{~Hz}$, and high start frequency point, $61 \mathrm{~Hz}$, were shifted to the right compared to the expected frequency-Watt curve. The inverter reaches the minimum point to export active power at $65 \mathrm{~Hz}$.

\subsubsection{Frequency-Watt testing with $61 \mathrm{~Hz}$ and $55 \mathrm{~Hz}$}

In order to test the performance of frequency-Watt control when the frequency is set as the minimum acceptable value, the following tests were conducted. Similar to the $58 \mathrm{~Hz}-61$ $\mathrm{Hz}$ tests, the following tests are also conducted with different time steps for voltages and voltage sweep directions.

\section{Frequency-Watt test sweep from low frequency to high frequency $(55 \mathrm{~Hz}-61 \mathrm{~Hz})$ with 2 seconds time step}

This test explores how well the frequency-Watt control performs when the frequency is set as the minimum acceptable value in the frequency-Watt function. The time step for frequency changes is 2 seconds in this test and the frequency sweep direction is from minimum to maximum. Within this test, the maximum export power was set as half of the maximum capacity of the inverter. The frequency set points of this test are summarized in the Table 6.19. The frequency point $\mathrm{f} 1$ is set as the low start frequency and point $\mathrm{f} 2$ is set as the high start frequency. Frequency point $\mathrm{f} 3$ is used for testing when the inverter exports the minimum power as the frequency higher than this value. As shown in Figure 6.28, the tested high start frequency point is about $62.5 \mathrm{~Hz}$ that was shifted to the right compared to the expected high start frequency point, $61 \mathrm{~Hz}$. 


\begin{tabular}{|c|c|c|c|}
\hline Frequency & Hz & P & Expected Watt \\
\hline f1 & 55 & W1 & 1650 \\
\hline f2 & 61 & W2 & 1650 \\
\hline f3 & 64 & W3 & 441 \\
\hline
\end{tabular}

Table 6.19: Frequency-Watt curve points with $58 \mathrm{~Hz}$ and $61 \mathrm{~Hz}$ sweep from the minimum to the maximum frequency.

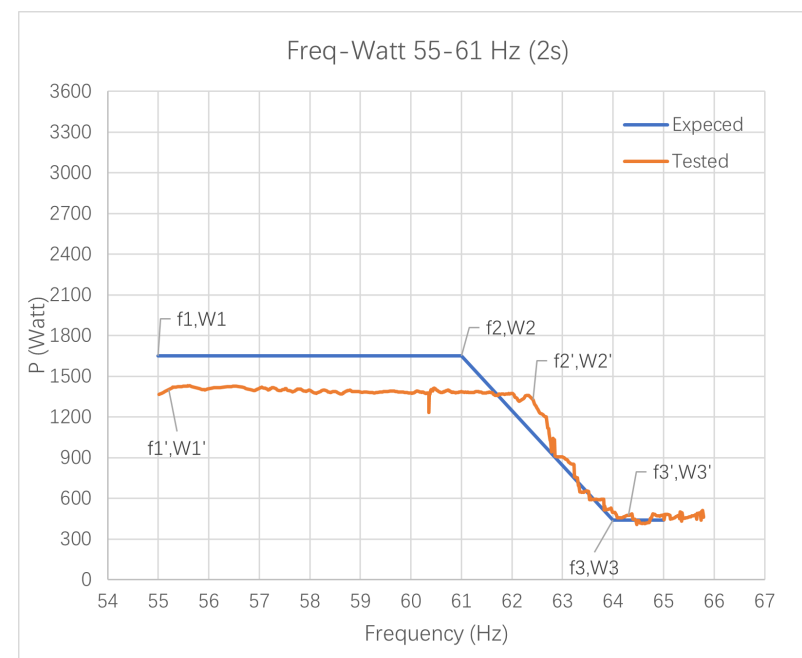

Figure 6.28: Frequency-Watt testing $(55 \mathrm{~Hz}-61 \mathrm{~Hz})$ sweep from low frequency to high frequency with 2 seconds time step and maximum power capacity.

The inverter exports the minimum active power at $64 \mathrm{~Hz}$.

\section{Frequency-Watt test sweep from high frequency to low frequency $(61 \mathrm{~Hz}-55 \mathrm{~Hz})$} with 2 seconds time step

Different from the previous test, the frequency sweep direction for this test was changed from maximum to minimum. The frequency step time in this test is still 2 seconds. Moreover, this test also explores the performance of the frequency-Watt control when maximum active power is provided. The frequency set points are summarized in the Table 6.7. 


\begin{tabular}{|c|c|c|c|}
\hline Frequency & Hz & P & Expected Watt \\
\hline f1 & 55 & W1 & 3300 \\
\hline f2 & 61 & W2 & 3300 \\
\hline f3 & 64 & W3 & 300 \\
\hline
\end{tabular}

Table 6.20: Frequency-Watt testing $(55 \mathrm{~Hz}-61 \mathrm{~Hz})$ sweep from low frequency to high frequency with 2 seconds time step and maximum power capacity.

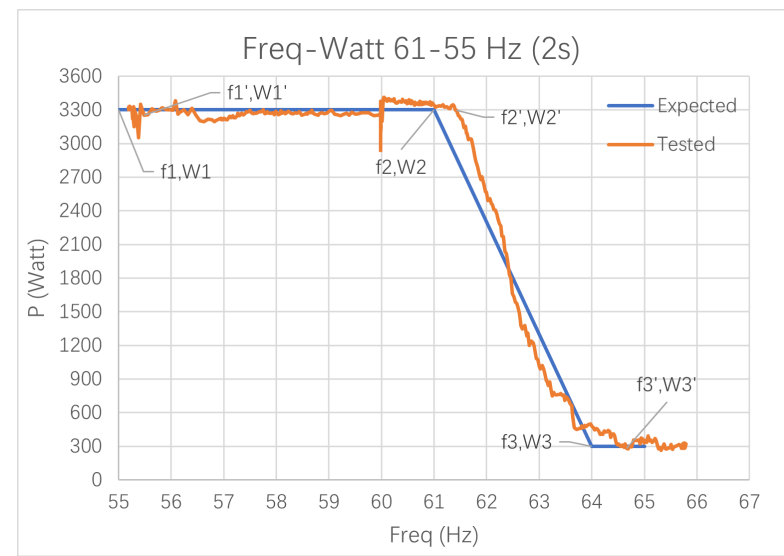

Figure 6.29: Frequency-Watt testing $(55 \mathrm{~Hz}-61 \mathrm{~Hz})$ sweep from low frequency to high frequency with 2 seconds time step and maximum power capacity.

As shown in Figure 6.28, the high start frequency was shifted to the right and the inverter exports the minimum power at around $64.5 \mathrm{~Hz}$, responding well to the frequency increase. The maximum export active power matches with the expected $3300 \mathrm{~W}$.

\section{Frequency-Watt test sweep from low frequency to high frequency $(55 \mathrm{~Hz}-61 \mathrm{~Hz})$} with 5 seconds time step

This test explores the performance of the frequency-Watt control as the frequencytime step extended to 5 seconds, and the frequency points set close to the minimum acceptable value. The frequency sweep direction of this test is from minimum to maximum. The frequency set points are summarized in Table 6.20. 


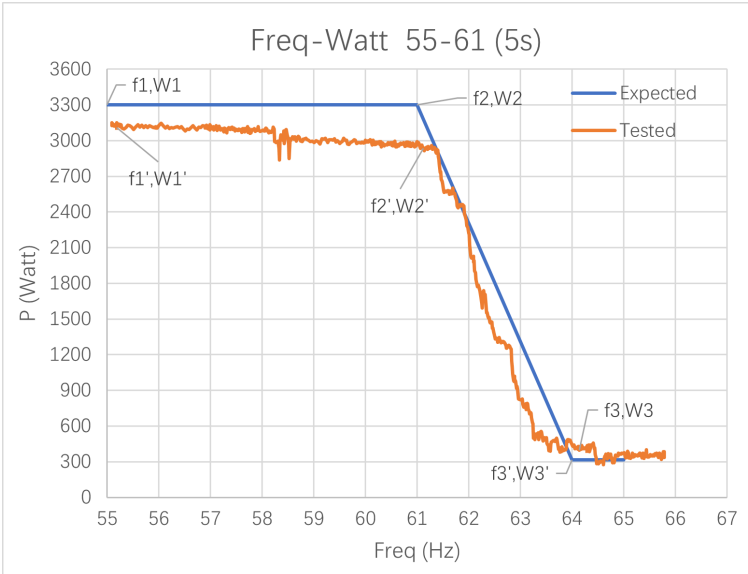

Figure 6.30: Frequency-Watt testing $(55 \mathrm{~Hz}-61 \mathrm{~Hz})$ sweep from low frequency to high frequency with 5 seconds time step and maximum power capacity.

As shown in Figure 6.30, the low start frequency point on the tested curve matches with the user defined point on the expected curve. The high start frequency point was shifted to the right. The inverter exports the minimum power at around $64.5 \mathrm{~Hz}$, responding to the frequency increase.

\section{Frequency-Watt test sweep from high frequency to low frequency $(61 \mathrm{~Hz}-55 \mathrm{~Hz})$ with 5 seconds time step}

This test explores the performance of the frequency-Watt control when the frequencysweep direction is from maximum to the minimum. The frequency time step was set as 5 seconds. The voltage set points are summarized in Table 6.20. 


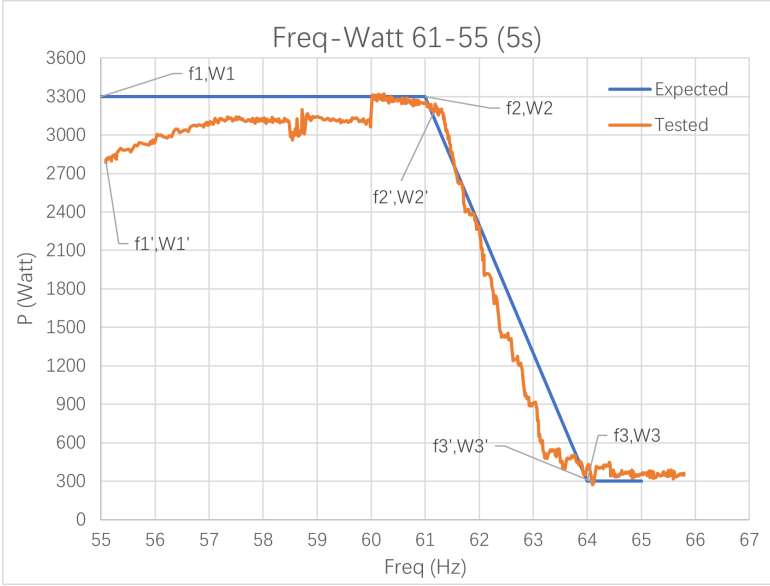

Figure 6.31: Frequency-Watt testing $(61 \mathrm{~Hz}-55 \mathrm{~Hz})$ sweep from high frequency to low frequency with 5 seconds time step and maximum power capacity.

As shown in Figure 6.31, the low frequency start point was shifted down and the high start frequency was shifted to the right. The inverter exports minimum power at 64 $\mathrm{Hz}$, responding to the frequency increase.

\subsubsection{Frequency-Watt testing with $65 \mathrm{~Hz}$ and $59.5 \mathrm{~Hz}$}

When the high start frequency on the frequency-Watt control curve is set close to the high frequency ride-through value, the inverter will not respond to the over-frequency disturbance because the frequency setting is too high for the grid frequency. In the US, the grid frequency is restricted to around $\pm 0.2 \%$ of $60 \mathrm{~Hz}$, so the stable grid frequency range is from 59.88 $\mathrm{Hz}$ to $60.12 \mathrm{~Hz}^{5}$. Such high frequency setting $(65 \mathrm{~Hz})$ is meaningless to the grid, but the tests conducted in this station explores the highest frequency value that can be put in the frequency-Watt function using the MATE3s and how well the inverter can follow the frequency-Watt curve. Similar to the test of low start frequency, $58 \mathrm{~Hz}$, and high start

\footnotetext{
${ }^{5}$ Depending on the specification of the balancing authority
} 
frequency, $61 \mathrm{~Hz}$, the followings tests are applied with different frequency sweep times and directions.

\section{Frequency-Watt test sweep from low frequency to high frequency $(59.5 \mathrm{~Hz}-65$}

\section{Hz) with 2 seconds time step}

This test explores how well the frequency-Watt control was conducted when the frequency points were set as the maximum acceptable value with frequency-time step 2 seconds and sweep direction from minimum to maximum. The frequency set points of this test are summarized in Table 6.8. The maximum export power in this test is half of the maximum capacity of the inverter. Frequency point $\mathrm{f} 2$ is the low start frequency, $59.5 \mathrm{~Hz}$, and the frequency point $\mathrm{f} 3$ is the high start frequency, $65 \mathrm{~Hz}$. The frequency point $\mathrm{fl}$ is used to explore how the frequency-Watt function works when the frequency is lower than the low start frequency. These three points apply to the rest of tests as well. The frequency set points are shown in Table 6.21.

\begin{tabular}{|c|c|c|c|}
\hline Frequency & Hz & P & Expected Watt \\
\hline f1 & 55 & W1 & 1650 \\
\hline f2 & 59.5 & W2 & 1650 \\
\hline f3 & 65 & W3 & 1650 \\
\hline
\end{tabular}

Table 6.21: Frequency-Watt testing $(59.5 \mathrm{~Hz}-65 \mathrm{~Hz})$ sweep from low frequency to high frequency with 2 seconds time step and half power capacity. 


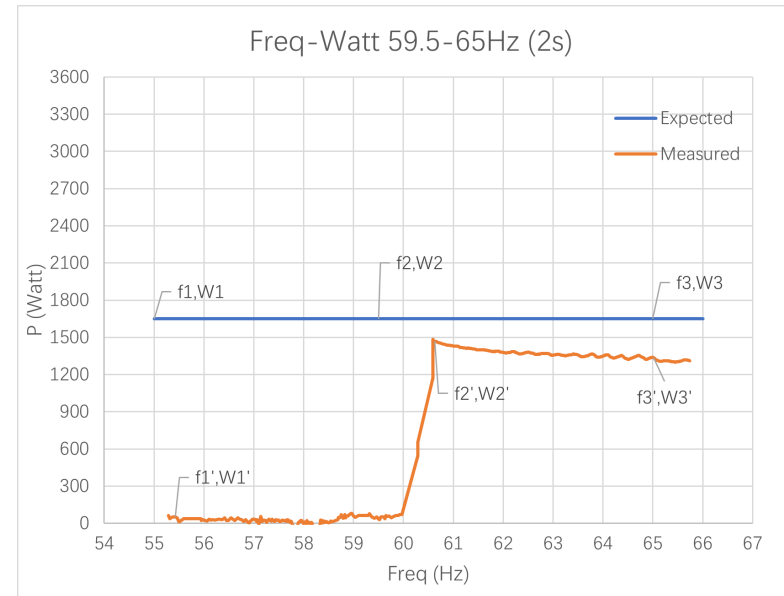

Figure 6.32: Frequency-Watt testing $(59.5 \mathrm{~Hz}-65 \mathrm{~Hz})$ sweep from low frequency to high frequency with $2 \mathrm{~s}$ and half power capacity.

As shown in Figure 6.32, the low start frequency point, $59.5 \mathrm{~Hz}$, is shifted to the right compared to the user-defined frequency. The inverter stops exporting maximum active power around $59.5 \mathrm{~Hz}$. The maximum export active power is lower than the expected value because the inverter was under discharging during the frequency-Watt test.

\section{Frequency-Watt test sweep from high frequency to low frequency $(65 \mathrm{~Hz}-59.5$}

\section{Hz) with 2 seconds time step}

Different from the previous test, the frequency-sweep direction of this test was changed to sweep from maximum to minimum. The frequency step time is set as 2 seconds. Moreover, this test also explores the performance of the frequency-Watt control with maximum acceptable frequency when the maximum active power capacity is provided. The voltage set points are summarized in the Table 6.22. 


\begin{tabular}{|c|c|c|c|}
\hline Frequency & Hz & P & Expected Watt \\
\hline f1 & 55 & W1 & 3000 \\
\hline f2 & 59.5 & W2 & 3000 \\
\hline f3 & 65 & W3 & 3000 \\
\hline
\end{tabular}

Table 6.22: Frequency-Watt testing $(65 \mathrm{~Hz}-59.5 \mathrm{~Hz})$ sweep from high frequency to low frequency with 2 seconds time step and full power capacity.

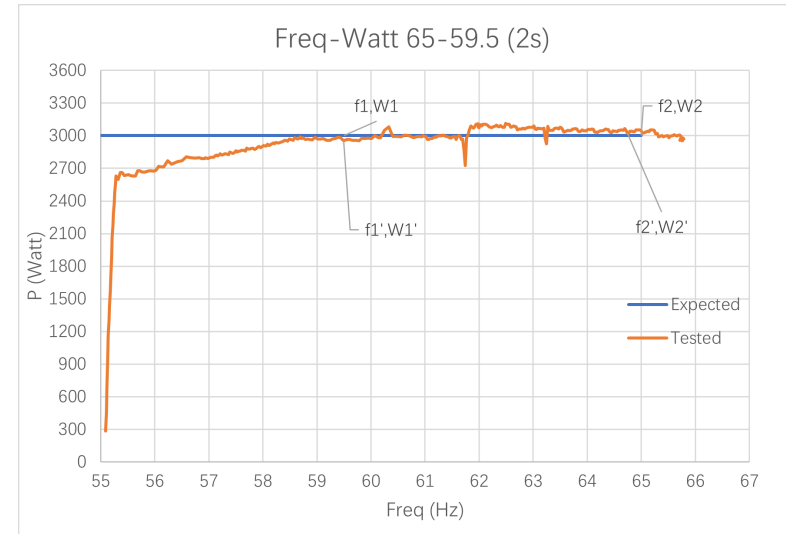

Figure 6.33: Frequency-Watt testing $(65 \mathrm{~Hz}-59.5 \mathrm{~Hz})$ sweep from high frequency to low frequency with 2 seconds time step and full power capacity.

Different than any other frequency-Watt testing, the inverter was trying to export the maximum power even through the frequency is lower than the low start frequency 59.5 $\mathrm{Hz}$, but without success. As shown in the Figure 6.33, the inverter started decreasing maximum export power at frequency is blow $59 \mathrm{~Hz}$.

\section{Frequency-Watt test sweep from low frequency to high frequency $(59.5 \mathrm{~Hz}-65$}

\section{Hz) with 5 seconds time step}

This test explores the performance of the frequency-Watt control as the frequency-time step extends to 5 seconds, and the frequency points were set close to the maximum acceptable values. The frequency sweep direction of this test is from minimum to maximum. The frequency set points are summarized in Table 6.22 


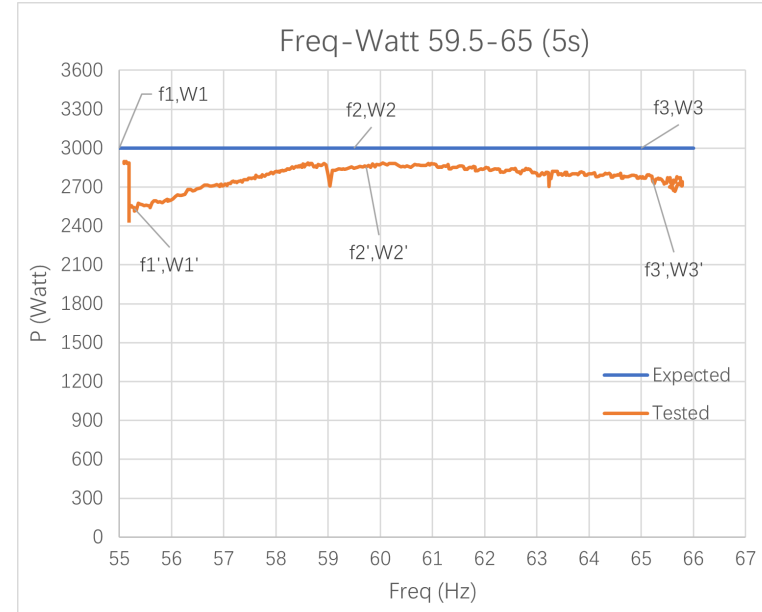

Figure 6.34: Frequency-Watt testing $(59.5 \mathrm{~Hz}-65 \mathrm{~Hz})$ sweep from low frequency to high frequency with 5 seconds time step and full power capacity.

As shown in Figure 6.34, the inverter starts decreasing the maximum power when the frequency is lower than $59.5 \mathrm{~Hz}$, but it was trying to export the maximum power.

4. Frequency-Watt test sweep from high frequency to low frequency $(65 \mathrm{~Hz}-59.5$ Hz) with 5 seconds time step

This test explores the performance of the frequency-Watt control when the frequencysweep direction was changed to sweep from maximum to minimum. The frequency time step was set as 5 seconds and the frequency set points are summarized in Table 6.22 . 


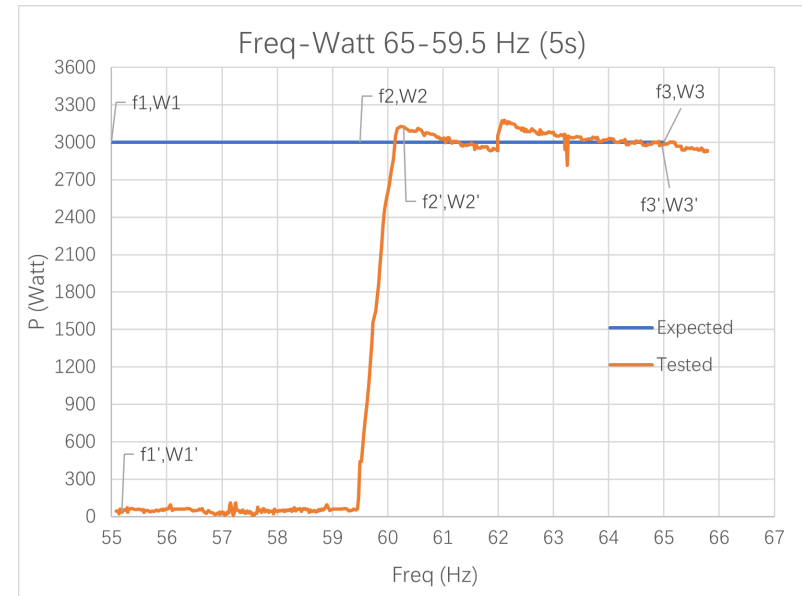

Figure 6.35: Frequency-Watt testing $(65 \mathrm{~Hz}-59.5 \mathrm{~Hz})$ sweep from high frequency to high frequency with $5 \mathrm{~s}$ time step and full power capacity.

Different than the previous two tests where the inverter was trying to export the maximum power, the inverter clearly stopped exporting the maximum power as the frequency dropped below $59.5 \mathrm{~Hz}$. When the frequency is between $59.5 \mathrm{~Hz}$ and $65 \mathrm{~Hz}$, the frequency-Watt control performs as expected, following the user-defined curve.

\subsubsection{Frequency-Watt function Sub-component Testing Analysis}

Based on the above testing result, the inverter testing system has the ability to test both the lower band and higher band of the frequency in the frequency-Watt function. The inverter has the ability to test the frequency-Watt function with different frequency sweep directions and is able to test the frequency-Watt function with different frequency time step changes even the GS 8048A smart inverter not always follow the user-defined curve very well. 


\section{Conclusion}

The motivation of this research was to develop a testing system for testing the interoperability of a grid-interactive smart inverter. The test system consists of three parts: send the IEEE 2030.5 messages to a DER controller and transfer the control parameters to the inverter; verify if the inverter can successfully receive and use those control parameters; use the grid simulator to test the ability of an inverter to follow the user-defined Volt-VAr and Frequency-Watt curve.

The smart inverter cannot directly accept use IEEE 2030.5 messages to operate its functions, so a DER client was created in the test system to convert the IEEE 2030.5 message to the control parameters for the inverter to use. The DER client is a program written in $\mathrm{C}++$ and running on a Raspberry $\mathrm{Pi}$. The DER client is able to receive the user-defined IEEE 2030.5 messages and transfer the messages to the control parameters. This is demonstrated by executing the DER program on the Raspberry Pi. However, not all the IEEE 2030.5 attributes can be successfully transferred since some SunSpec registers in the AXS port of the GS8048A smart inverter have not been implemented. The DER client controls the inverter through the AXS port because the inverter is a register-controlled device and the AXS port is the collection of the SunSpec registers to control the inverter.

In order to verify if the inverter receives the control parameters or not, the Outback Shell program and Modbus Poll are used. These two methods read the status of the SunSpec 
registers as long as the IP address of the AXS port can be figured. Based on the test result of using the Outback Shell program and Modbus Poll in the analysis section, some values defined in the IEEE 2030.5 were successfully transferred. The values that cannot be transferred are because not all the IEEE 2030.5 attributes to the inverter have a relevant SunSpec register to use. Some SunSpec registers have not been developed or implemented in the AXS port for the GS8048A smart inverter.

To show if the inverter has the ability to follow the user-defined control curve, the grid simulator is used. The grid simulator provides variable voltage and frequency changes for the inverter to enable and operate with Volt-VAr and frequency-Watt function. As many test results show in the analysis section, the GS8048A smart inverter sometimes cannot correctly follow the user-defined Volt-VAr and frequency-Watt curves. The test system developed for this thesis research is a success since the test system has the ability to test different functions of an inverter.

In all, the testing system designed for the grid-interactive smart inverter can perform tests for such inverters to meet the relevant IEEE 2030.5 functionality. 


\section{Bibliography}

[1] J. G. Kassakian, W. M. Hogan, R. Schmalensee, and H. D. Jacoby. The future of the electric grid, Boston, MA, USA: MIT Press 2011.

[2] Goran Strbac. Demand side management: Benefits and challenges. Energy Policy, 36:4419-4426, November 2008. [Online].

[3] Noriko Kawakami and Yukihisa Iijima. Overview of battery energy storage systems for stabilization of renewable energy in Japan. In 2012 International Conference on Renewable Energy Research and Applications (ICRERA), pages 1-5, 2012.

[4] Matthew T. Lawder, Bharatkumar Suthar, Paul W. C. Northrop, Sumitava De, C. Michael Hoff, Olivia Leitermann, Mariesa L. Crow, Shriram Santhanagopalan, and Venkat R. Subramanian. Battery energy storage system (BESS) and battery management system (BMS) for grid-scale applications. Proceedings of the IEEE, 102(6):1014-1030, 2014.

[5] Aaron Larson. What is DERMS and how can it help utilities? POWER Magazine, July. 08 2020. [Online]. 
[6] Office of Energy Efficiency and Renewable Energy. Using distributed energy resources: A how-to guide for federal facility managers. Cogeneration and Competitive Power Journal, 17:4(2), 2002. [Online].

[7] U.S. Energy Information Administration. Battery storage in the United States: An update on market trends. Technical report, Washington, DC, USA, 2020. [Online].

[8] Kelsey Misbrener. Smart inverters redefine relationship between DERs and the grid. Solar Power World, March. 12 2019. [Online].

[9] Microgrid Knowledge Editors. How DERMS and smart inverters safely bring distributed resources to the grid. Microgrid Knowledge, Jun. 25 2018. [Online].

[10] O.I. Adekola and A.K. Raji. Functionalities of smart inverter system for grid-connected applications. In 2015 International Conference on the Industrial and Commercial Use of Energy (ICUE), pages 340-344, 2015.

[11] Gordon Lum. California use case for IEEE 2030.5 for distributed energy renewables. IEEE newsletter, Dec 2016 [Online].

[12] Lloyd Cibulka, Tom Tansy, Bob Fox, Suzanne Martinez, Dr. Jan Kleissl, and Edmund G. Brown Jr. Smart inverter performance testing to UL 1741 SA and CA Rule 21 criteria. RAND Corp., Santa Monica, CA, USA, RR-1776-NYCEDC, 2018 [Online].

[13] SunSpec Orgnazation. Common Smart Inverter Profile, March, 2018. 
[14] Califonia Public Utilities Commission. SIWG Phase 3 DER Functions: Recommendations to the CPUC for Rule 21, Phase 3 Function Key Requirements, and Additional Discussion Issues, March 31, 2017.

[15] Stuart Mccafferty. Creating DER integration solution architectures using IEEE 2030.5 and the energy IoT common reference model. GridIntellect, LLC in the Digital Utility Group.

[16] Frédéric Wauquiez. IEEE 2030.5 - connect to the wide world of distributed energy resources. General Electric Digital website, Aug. 5 2020. [Online].

[17] James Obert, Patricia Cordeiro, Jay Johnson, Gordon Lum, Tom Tansy, Max Pala, and Ronald Ih. Recommendations for trust and encryption in DER interoperability standards, 022019.

[18] Outback Power Company. Radian series inverter/charger GS8048 operator's manual, Oct 2013. [Online].

[19] Outback Power Technologies. MATE3s Advanced System Display and Communications. Arlington, WA, USA, 2019 [Online].

[20] Outback Power Technologies. AXS port sunspec modbus interface owner's manual, April 2016. [Online].

[21] Inc Aquion Energy. Operations manual battery module model M100-LS082, 2016. 
[22] NH Research, Inc, Irvine, California. NHR 94109420 Series User's Manual, L edition, June 20, 2019.

[23] Tektronix. Power Analyzer PA4000 Datasheet. Portland, OR, USA, February 2016.

[24] Tektronix. TPS2000B Digital Storage Oscilloscopes. Portland, OR, USA, October 2018.

[25] IEEE standards association. IEEE standard for smart energy profile application protocol. IEEE Std 2030.5-2018 (Revision of IEEE Std 2030.5-2013), pages 1-361, 2018.

[26] James Mater, Steve Kang, and Robby Simpson. IEC 61850 and IEEE 2030.5: A Comparison of 2 Key Standards for DER Integration: An Update. PacWorld, 2019. [Online].

[27] Outback Power Technologies. Mate3s Overview Guide. Arlington, WA, USA, November 2019.

[28] Dr. Meter Inc. PS305DM-DC bench power supply user manual. Dr. Meter website.

[29] Tektronix. PWRVIEW Online Help. Tektronix, Inc, Beaverton, OR, USA, March 2016. [Online].

[30] Andy Hoke, Austin Nelson, Jin Tan, Vahan Gevorgian, Chris Antonio, Ken Fong, Mohamed Elkhatib, Jay Johnson, Rasel Mahmud, Jason Neely, and Dean Arakawa. The 
Frequnecy-Watt Function Simulation and Testing for the Hawaiian Electric Companies. NREL goverment official website, 2017 [Online].

[31] Karl Stein, Moe Tun, Marc Matsuura, and Richard Rocheleau. Characterization of a Fast Battery Energy Storage System for Primary Frequency Response. Energies, 11(12), 2018. 